\title{
ERS statement on chronic thromboembolic pulmonary hypertension
}

\author{
Marion Delcroix (101,2,29 , Adam Torbicki (103,27, Deepa Gopalan ${ }^{4,27}$, Olivier Sitbon ${ }^{5,27}$, \\ Frederikus A. Klok (1) ${ }^{6,27}$, Irene Lang ${ }^{7,27}$, David Jenkins ${ }^{8,27}$, Nick H. Kim, ${ }^{9,2}$, \\ Marc Humbert (10 ${ }^{5,27}$, Xavier Jais ${ }^{5,27}$, Anton Vonk Noordegraaf (10 10,27 , \\ Joanna Pepke-Zaba ${ }^{8,27}$, Philippe Brénot ${ }^{11}$, Peter Dorfmuller ${ }^{12,13,14}$, Elie Fadel ${ }^{15}$, \\ Hossein-Ardeschir Ghofrani (1012,13,14, Marius M. Hoeper (15 ${ }^{15}$, Pavel Jansa ${ }^{16}$, \\ Michael Madani ${ }^{17}$. Hiromi Matsubara (18), Takeshi Ogo ${ }^{19}$, Ekkehard Grünig ${ }^{20}$ \\ Andrea D'Armini ${ }^{21}$, Nazzareno Galie ${ }^{22}$, Bernhard Meyer ${ }^{23}$, Patrick Corkery ${ }^{24,30}$, \\ Gergely Meszaros ${ }^{25,30}$, Eckhard Mayer ${ }^{26,28,29}$ and Gérald 'Simonneau 5,28,29
}

@ERSpublications

The ERS statement on chronic thromboembolic pulmonary hypertension outlines a review of the literature and current practice concerning diagnosis and management of CTEPH https://bit.ly/34dIjot

Cite this article as: Delcroix M, Torbicki A, Gopalan D, et al. ERS statement on chronic thromboembolic pulmonary hypertension. Eur Respir J 2021; 57: 2002828 [https://doi.org/10.1183/13993003.02828-2020].

ABSTRACT Chronic thromboembolic pulmonary hypertension (CTEPH) is a rare complication of acute pulmonary embolism, either symptomatic or not. The occlusion of proximal pulmonary arteries by fibrotic intravascular material, in combination with a secondary microvasculopathy of vessels $<500 \mu \mathrm{m}$, leads to increased pulmonary vascular resistance and progressive right heart failure. The mechanism responsible for the transformation of red clots into fibrotic material remnants has not yet been elucidated. In patients with pulmonary hypertension, the diagnosis is suspected when a ventilation/perfusion lung scan shows mismatched perfusion defects, and confirmed by right heart catheterisation and vascular imaging. Today, in addition to lifelong anticoagulation, treatment modalities include surgery, angioplasty and medical treatment according to the localisation and characteristics of the lesions.

This statement outlines a review of the literature and current practice concerning diagnosis and management of CTEPH. It covers the definitions, diagnosis, epidemiology, follow-up after acute pulmonary embolism, pathophysiology, treatment by pulmonary endarterectomy, balloon pulmonary angioplasty, drugs and their combination, rehabilitation and new lines of research in CTEPH.

It represents the first collaboration of the European Respiratory Society, the International CTEPH Association and the European Reference Network-Lung in the pulmonary hypertension domain. The statement summarises current knowledge, but does not make formal recommendations for clinical practice.

The institutions and patient representatives associated with affiliation numbers $1-5,8,10,12-16$ and 22-25 are part of the European Reference Network for rare lung diseases (ERN-Lung).

This ERS statement was endorsed by the ERS Executive Committee on 19 Nov 2020, by ERN-LUNG on 30 Nov 2020 and by the International CTEPH Association (ICA) on 07 Dec 2020

This article has supplementary material available from erj.ersjournals.com

Received: 17 July 2020 | Accepted: 5 Nov 2020

Copyright @ERS 2021. For reproduction rights and permissions contact permissions@ersnet.org 


\section{Scope of document}

Since the publication of the 2015 European Society of Cardiology (ESC)/European Respiratory Society (ERS) guidelines for the diagnosis and treatment of pulmonary hypertension ( $\mathrm{PH})$ [1], considerable advances have been made in the understanding, diagnosis and management of chronic thromboembolic pulmonary hypertension (CTEPH). Thus, the ERS considered a statement paper was required in order to take into account recent developments in the field. A multidisciplinary task force, with members from the ERS, the International CTEPH Association (ICA) and the European Reference Network (ERN)-Lung, including specialists in pneumology, cardiology, cardiothoracic surgery, radiology and pathology, together with patient representatives, defined and answered key questions related to the management of CTEPH. The task force searched for a clear definition; reviewed imaging techniques, epidemiology, follow-up of acute pulmonary embolism (PE), microvasculopathy, indications of pulmonary endarterectomy (PEA), balloon pulmonary angioplasty (BPA) and medical treatment and the multimodality approach; and defined research questions and priorities.

\section{Methods}

Task force chairs, supported by the ICA board members, compiled a list of 11 topics that they considered important and relevant to the diagnosis and management of CTEPH. Task force members (27 experts and two patient representatives) were divided into subgroups targeting these topics (supplementary table S1). Each group identified three or four key focused questions relevant for their topic. These were discussed in a first face-to-face meeting in March 2019. Once the key questions were agreed, the subgroups prepared individual subsections which were then presented and discussed in a second face-to-face meeting during the ERS congress in Madrid (September 2019) and subsequently revised until consent among all co-authors was reached (February 2020). All co-authors critically revised and approved the final statement (March 2020). The manuscript was finalised and submitted to the European Respiratory Journal after approval by the ERS Guidelines Committee (April-May 2020). The statement was presented at the ERS virtual International Congress (September 2020).

The present ERS statement combines an evidence-based approach with the clinical expertise of the task force members, based on both literature search and discussions during face-to-face meetings. The task force members reviewed current knowledge and new scientific advances through identifying relevant individual studies and reviews from systematic searches in MEDLINE (via PubMed) and Embase (via Ovid), performed by subgroup members, mostly with no date limits. The last search was performed in February 2020; however, task force members also tracked any relevant citation which appeared beyond this date and until submission in April 2020. The search was limited to English literature with no date limitations. Literature search was conducted using "chronic thromboembolic pulmonary hypertension" and the relevant key words for each respective section (supplementary table S2). Additionally, manual searches of articles mentioned in the reference lists were performed.

Affiliations: ${ }^{1}$ Clinical Dept of Respiratory Diseases, Pulmonary Hypertension Center, UZ Leuven, Leuven, Belgium. ${ }^{2}$ BREATHE, Dept CHROMETA, KU Leuven, Leuven, Belgium. ${ }^{3}$ Dept of Pulmonary Circulation, Thrombo-embolic Diseases and Cardiology, Center of Postgraduate Medical Education, ECZ-Otwock, Otwock, Poland. ${ }^{4}$ Dept of Radiology, Imperial College Hospitals NHS Trusts, London, UK. ${ }^{5}$ Université Paris-Saclay; Inserm UMR_S 999, Service de Pneumologie, Hôpital Bicêtre (AP-HP), Le Kremlin-Bicêtre, France. ${ }^{6}$ Dept of Medicine - Thrombosis and Hemostasis, Leiden University Medical Center, Leiden, The Netherlands. ${ }^{7}$ Medical University of Vienna, Vienna, Austria. ${ }^{8}$ Royal Papworth Hospital, Cambridge University Hospital, Cambridge, UK. ${ }^{9}$ Division of Pulmonary, Critical Care, and Sleep Medicine, University of California San Diego, La Jolla, CA, USA. ${ }^{10}$ Dept of Pulmonary Medicine, Amsterdam UMC, Vrije Universiteit Amsterdam, Amsterdam Cardiovascular Sciences, Amsterdam, The Netherlands. ${ }^{11}$ Marie Lannelongue Hospital, Paris-South University, Le Plessis Robinson, France. ${ }^{12}$ University of Giessen and Marburg Lung Center, German Center of Lung Research (DZL), Giessen, Germany. ${ }^{13}$ Dept of Medicine, Imperial College London, London, UK. ${ }^{14}$ Dept of Pneumology, Kerckhoff-Clinic Bad Nauheim, Bad Nauheim, Germany. ${ }^{15}$ Hannover Medical School, Hannover, Germany. ${ }^{16}$ 2nd Department of Medicine, Dept of Cardiovascular Medicine, First Faculty of Medicine, Charles University and General University Hospital in Prague, Prague, Czech Republic. ${ }^{17}$ Sulpizio Cardiovascular Centre, University of California, San Diego, CA, USA. ${ }^{18}$ National Hospital Organization Okayama Medical Center, Okayama, Japan. ${ }^{19}$ National Cerebral and Cardiovascular Centre, Osaka, Japan. ${ }^{20}$ Thoraxklinik Heidelberg at Heidelberg University Hospital, Heidelberg, Germany. ${ }^{21}$ Unit of Cardiac Surgery, Intrathoracic Transplantation and Pulmonary Hypertension, University of Pavia School of Medicine, Foundation I.R.C.C.S. Policlinico San Matteo, Pavia, Italy. ${ }^{22}$ University of Bologna, Bologna, Italy. ${ }^{23}$ Institute for Diagnostic and Interventional Radiology, Hannover Medical School, Hannover, Germany. ${ }^{24} \mathrm{PHA}$ Ireland, Dublin, Ireland. ${ }^{25} \mathrm{PHA}$ Europe, Hungary. ${ }^{26}$ Dept of Thoracic Surgery, Kerckhoff Clinic Bad Nauheim, Bad Nauheim, Germany.

${ }^{27}$ Section editors. ${ }^{28}$ Equal contribution. ${ }^{29} \mathrm{Co}$-chair. ${ }^{30}$ Patient representative.

Correspondence: Marion Delcroix, Dept of Respiratory Diseases, University Hospitals of Leuven, Herestraat 49, 3000 Leuven, Belgium. E-mail marion.delcroix@uzleuven.be 


\section{BOX 1 Summary of statements}

\section{Definitions}

1.1 All patients in whom symptoms can be attributed to post-thrombotic deposits within pulmonary arteries could be considered to have CTEPD with or without PH. CTEPH remains the preferred term in patients with $\mathrm{PH}$.

1.2 CPET and exercise-RHC could contribute to the definition of CTEPD helping to identify the main cause of exercise limitation in the presence of comorbidities and in patients without resting $\mathrm{PH}$.

1.3 In many patients with CTEPH, resting mPAP is normalised by surgical or multimodality treatment and patients feel healthy, but it is unlikely to return all pulmonary vessels back to normal.

\section{Diagnosis}

2.1 $V^{\prime} / Q^{\prime}$ scintigraphy remains the most effective screening tool to exclude CTEPD. $V^{\prime} / Q^{\prime}$ SPECT has been shown to be superior to planar imaging and is the methodology of choice. Because the transition from planar imaging to SPECT may not be easy for clinicians unfamiliar with the three-dimensional anatomy, two-dimensional planar images can be reprojected from SPECT without losing diagnostic accuracy.

2.2 Alternative perfusion techniques such as dual energy computed tomography and magnetic resonance perfusion have numerous theoretical advantages over $V^{\prime} / Q^{\prime}$, but are more technically challenging and expensive, with limited availability and lacking multicentre validation. Hence, they are not replacing $V^{\prime} / Q^{\prime}$ in current clinical practice.

2.3 High-quality CTPA is adequate for diagnosis of proximal CTEPH, but a negative CTPA, even if high-quality, does not exclude CTEPH, as distal disease can be missed.

2.4 Cone beam computed tomography and area detector computed tomography allow for more accurate visualisation of subsegmental vasculature and have been shown to be useful for procedural guidance for BPA. The benefits of the technology require validation in prospective trials before recommendation for routine clinical use.

2.5 MRI is not yet fully integrated into the CTEPH diagnostic algorithm, because its use is heavily dependent on local practice and is routinely implemented only in a few high-volume institutions where there is clustering of expertise.

\section{Epidemiology}

3.1 CTEPH is a rare and underdiagnosed complication of PE and, upon first presentation, may be misclassified as acute PE.

3.2 Persistent perfusion defects after acute PE are common, but have highly variable clinical relevance, ranging from completely asymptomatic to established CTEPH.

3.3 We have no evidence to suggest that CTEPD without PH is an early stage of CTEPH, because of a lack of data on its natural history.

3.4 Current literature could not demonstrate any difference in CTEPH incidence in patients with venous thromoboembolism treated with VKAs and NOACs, but rigorous studies have not been done.

3.5 Screening for CTEPH in asymptomatic patients with specific risk factors could be effective, but is not supported by any data.

\section{Follow-up after acute $\mathrm{PE}$}

4.1 Earlier diagnosis of CTEPH may be relevant for patient outcomes. It can be achieved by targeting patients with acute PE with risk factors for CTEPH or with radiological findings suggesting CTEPH.

4.2 Patients at risk of CTEPH can be identified by accurate assessment of the CTPA images used to diagnose PE, individual risk factors for $\mathrm{CTEPH}$, and symptoms of functional limitations and/or right heart failure in the course of PE.

4.3 Echocardiography is the test of choice in patients with suspected CTEPH. Other tests such as Leiden CTEPH rule-out criteria, $V^{\prime} / Q^{\prime}$ scan or CPET could be used to exclude the presence of CTEPH and/or to establish an alternative diagnosis.

4.4 The optimal timing for considering CTEPH in patients with PE is at the routine 3-month follow-up visit, but earlier work-up may be necessary in highly symptomatic or deteriorating patients.

\section{Pathophysiology}

5.1 Current knowledge suggests that two types of vascular lesions exist in CTEPH: proximal fibrotic obstruction in large elastic pulmonary arteries and a secondary microvasculopathy in pulmonary vessels $<500 \mu \mathrm{m}$.

5.2 Morphological delineation of pulmonary and bronchial circulation is possible with CTPA, while MRI permits both anatomical visualisation and semi-quantitative analysis of the extent of bronchopulmonary shunting. None of these techniques can quantify the microvasculopathy.

5.3 The right ventricle is less adapted in CTEPH than in idiopathic PAH, but recovers largely after successful PEA.

\section{PEA}

6.1 Requirements for a PEA centre are based on expert opinion only, but $\geqslant 50$ cases as centre annual volume, and a properly trained surgeon have been proposed.

6.2 There is probably considerable anatomical overlap between BPA and PEA, but segmental and subsegmental disease are suitable for surgery in expert, high-volume centres.

6.3 Successful outcome after PEA is multifactorial and assumes in-hospital mortality $<5 \%$, survival of $90 \%$ at 3 years, improved functional class and quality of life.

6.4 Residual PH after PEA presents in 50\% and represents a target for recently introduced medical therapies and BPA. There is some evidence for their use if post-PEA mPAP remains $>30 \mathrm{mmHg}$ in the presence of symptoms.

\section{BPA}

7.1 Inoperable CTEPH patients can benefit from BPA. Optimal CTEPH treatment requires a multidisciplinary team approach considering PEA, medical therapy and BPA.

7.2 Long-term results after BPA are available out to 8 years after the procedure. Safety and efficacy of BPA correlate with centre experience.

7.3 Vascular injury rather than reperfusion pulmonary oedema is the likely cause of any severity of lung injury after BPA.

7.4 Similar to PEA, proper training in a high-volume centre is critical for BPA.

\section{Medical treatment}

8.1 According to current guidelines, CTEPH patients should be treated with lifelong anticoagulation; VKAs are the mainstay of anticoagulant treatment in CTEPH, but NOACs are increasingly used, with no safety issues reported yet. Antiphospholipid syndrome is a contraindication to NOACs. 


\section{Continued}

8.2 Riociguat, an oral guanylate cyclase stimulator, and treprostinil, a subcutaneous prostacyclin analogue, are approved for patients with inoperable CTEPH or persistent/recurrent PH after PEA; other PH medications have been tested in CTEPH and are used off-label.

8.3 Oral combination therapy is a common practice in CTEPH patients with severe haemodynamic compromise.

8.4 Withdrawal of PH medications is usually considered after successful BPA and/or PEA.

\section{Multimodal treatment}

9.1 There is no consensus on eligibility criteria for multimodal therapy, which is dependent on the expertise and judgement of each individual CTEPH centre. Patient selection for multimodal therapy is performed in expert centres through a multidisciplinary team approach.

9.2 There is no convincing evidence that pre-operative pharmacological treatment is beneficial in patients with operable CTEPH. Nevertheless, PH-targeted drugs as bridging therapy to PEA is sometimes considered in selected high-risk patients (with high preoperative pulmonary vascular resistancel after multidisciplinary team assessment in expert centres.

9.3 The role of BPA as bridging therapy prior to PEA in patients with mixed surgically accessible and inaccessible lesions needs to be explored. The potential benefit of hybrid procedures simultaneously combining PEA and BPA in such patients needs to be confirmed by further studies. There is insufficient evidence to support the use of BPA as a rescue procedure for early failure of PEA. Additional BPA, most often in combination with PH-targeted medical therapy, is common practice for patients with persistent symptomatic PH after PEA.

9.4 Most task force members use PH-targeted therapy prior to BPA to improve the safety of BPA, although there have been no clinical trials investigating the benefit of this approach on the rate of BPA-related complications. Some data suggest that the combination of $\mathrm{PH}$-targeted therapy and BPA provides better results than medical therapy alone.

\section{Rehabilitation}

10.1 Although exercise physiology is slightly different in CTEPH in comparison to PAH, it is reasonable to believe that the positive effects of rehabilitation are similar for both conditions.

10.2 Rehabilitation seems to be effective and safe in inoperable CTEPH patients.

10.3 Although most of the pivotal trials on rehabilitation in CTEPH and PAH were based on in-hospital training in tertiary referral set-ups, small nonrandomised studies have shown that home-based rehabilitation might also be an effective and safe option.

10.4 A carefully monitored, low-dose rehabilitation programme of CTEPH patients after PEA or BPA might be considered standard of care.

\section{Global research lines}

11.1 To achieve new genetic insights and basic science progress, international collaboration through biobanking should be established to allow researchers access to the data which represents a large number of patients. Integration of clinical and -omic measurements will identify clinically valuable diagnostic signatures and pathways for potential future interventions.

11.2 There is a need to address CTEPH in paediatric patients by a prospective global registry.

CTEPD: chronic thromboembolic pulmonary disease; $\mathrm{PH}$ : pulmonary hypertension; CTEPH: chronic thromboembolic pulmonary hypertension CPET: cardiopulmonary exercise testing; RHC: right heart catheterisation; mPAP: mean pulmonary arterial pressure; $V^{\prime} / Q^{\prime}$ : ventilation/ perfusion; SPECT: single photon emission computed tomography; CTPA: computed tomography pulmonary angiography; BPA: balloon pulmonary angioplasty; MRI: magnetic resonance imaging; PE: pulmonary embolism; VKA: vitamin K antagonist; NOAC: non-vitamin $\mathrm{K}$ antagonist oral anticoagulant; PAH: pulmonary arterial hypertension; PEA: pulmonary endarterectomy.

All members of the task force disclosed their conflicts of interest before initiation of the project and upon submission of the manuscript.

\section{Definitions}

\subsection{Current definition: is there a need to change it in view of new diagnostic methods and the} proposed reduced mean pulmonary arterial pressure threshold for PH? How to best define symptomatic patients with post-thrombotic deposits but without PH at rest?

Two terms are currently used to describe symptomatic patients with chronic thromboembolic occlusions of pulmonary arteries according to the presence or the absence of $\mathrm{PH}$ at rest: chronic thromboembolic pulmonary hypertension (CTEPH) and chronic thromboembolic disease (CTED).

The definition provided in the 2015 ESC/ERS guidelines for the diagnosis and treatment of pulmonary hypertension [1] stated that "The diagnosis of CTEPH is based on findings obtained after at least 3 months of effective anticoagulation in order to discriminate this condition from 'subacute' PE. These findings are mPAP $\geqslant 25 \mathrm{mmHg}$ with PAWP $\leqslant 15 \mathrm{mmHg}$, mismatched perfusion defects on lung scan and specific diagnostic signs for CTEPH seen by multidetector CTPA, MRI or conventional pulmonary cineangiography, such as ring-like stenoses, webs/slits and chronic total occlusions (pouch lesions or tapered lesions)". More recently, cohorts of symptomatic patients, with exercise limitation, have been described showing similar obstructive lesions as CTEPH but no resting $\mathrm{PH}[2,3]$. However, they may benefit from the same treatment, including lifelong anticoagulation, PEA and BPA. The reporting authors proposed the term "chronic thromboembolic disease (CTED)", which is unclear because it does not specify that it concerns pulmonary vessels.

The only difference between CTEPH and CTED is the presence or not of PH at rest. However, based on scientific evidence, a change in the definition of $\mathrm{PH}$ has been proposed with a decrease of the threshold mean pulmonary arterial pressure (mPAP) from 25 to $21 \mathrm{mmHg}$, and in the definition of pre-capillary $\mathrm{PH}$ 
with a decrease of the threshold pulmonary vascular resistance (PVR) from 3 to 2 Wood units [4]. These lowered thresholds, if adopted in upcoming guidelines, may influence the designation of former CTED patients as CTEPH patients [2, 5-9] (supplementary table S3).

To solve this terminology dilemma, a survey was conducted among the task force members. After collation of all propositions for names of the overarching disease and the subentities with or without $\mathrm{PH}$, a three-round voting process was conducted until consensus was reached. For all symptomatic patients who present mismatched perfusion defects on ventilation/perfusion $\left(V^{\prime} / Q^{\prime}\right)$ scan and specific signs of organised fibrotic clots on computed tomography pulmonary angiography (CTPA), magnetic resonance imaging (MRI) or conventional pulmonary cineangiography, such as ring-like stenoses, webs/slits and chronic total occlusions (pouch lesions or tapered lesions), after $\geqslant 3$ months of effective anticoagulation, the task force chose the general term "chronic thromboembolic pulmonary disease (CTEPD)". Some of these patients have no $\mathrm{PH}$ at rest whatever the definition accepted. For the others, the term "chronic thromboembolic pulmonary hypertension (CTEPH)" was maintained. The presence of $\mathrm{PH}$ at rest in this setting is not only the consequence of proximal vessel obstruction by organised thrombi, but can also be related to a secondary microvasculopathy, as well as to the presence of underlying lung or left heart disease.

\subsection{Should cardiopulmonary exercise testing and exercise-right heart catheterisation be included in the definition and with what thresholds?}

Since symptoms in CTEPH appear during exercise, the tests performed at rest, including right heart catheterisation (RHC), may lack sensitivity. Long-awaited recently formulated criteria for exercise $\mathrm{PH}$ are not optimal for detection of "exercise CTEPH", as they take into account total PVR (mPAP/cardiac output) and not PVR, ignoring pulmonary artery wedge pressure (PAWP) changes at exercise [10]. However, reliable measurement of PAWP at peak exercise may be difficult due to large respiratory shifts in intrathoracic pressure. Much attention is given to cardiopulmonary exercise testing (CPET) performed not only for prognostic purposes, but for differential diagnosis of dyspnoea in patients with suspected pulmonary vascular disease [11-15]. The candidate CPET diagnostic parameters are reflecting inefficient ventilation and include high ventilatory equivalent for carbon dioxide (minute ventilation/carbon dioxide production) slope and low end-tidal carbon dioxide tension, further decreasing at exercise [16]. Both are promising for the differential diagnosis of symptomatic patients without or with only mild $\mathrm{PH}$ who have imaging results that may suggest CTEPD. In addition, it is not clear whether CTEPH can be diagnosed in the presence of suggestive intravascular changes at imaging but in patients who also have increased left ventricular (LV) filling pressures. Whether treatment removing pre-capillary obstruction would lead to pulmonary congestion or, in contrast, would improve cardiorespiratory reserve by restoring flow through ventilated, but underperfused lung areas, remains unclear.

New imaging tools and increasing experience of radiologists in CTEPH referral centres make it possible to identify patients with chronic thromboembolic obstructions clinically presenting as acute PE or with long-standing progressive exercise limitation. The definition of CTEPH required 3 months of anticoagulation. This is certainly still reasonable in haemodynamically stable patients and those who clearly improve during initial anticoagulant therapy. However, in individual severely compromised cases with significant contribution of unequivocal chronic intravascular changes, the diagnosis and specific treatment for CTEPH could be considered by experienced CTEPH teams, regardless of the duration of anticoagulant therapy.

\subsection{Can a patient be cured of CTEPH?}

Despite optimal, and apparently successful, surgical, interventional and medical treatment, pulmonary circulation does not return entirely to normal, which can be seen at $V^{\prime} / Q^{\prime}$ scans performed during follow-up. Thus, treatment may make patients with CTEPH feel healthy, but is unlikely to completely cure all the affected pulmonary vessels: arteries, veins and systemic collaterals [17, 18]. Furthermore, the possibility to restore normal life expectancy is somewhat limited by potential complications related to lifelong anticoagulation.

\section{Statements: definitions}

1.1 All patients in whom symptoms can be attributed to post-thrombotic deposits within pulmonary arteries could be considered to have CTEPD with or without PH. CTEPH remains the preferred term in patients with $\mathrm{PH}$.

1.2 CPET and exercise-RHC could contribute to the definition of CTEPD helping to identify the main cause of exercise limitation in the presence of comorbidities and in patients without resting $\mathrm{PH}$.

1.3 In many patients with CTEPH, resting mPAP is normalised by surgical or multimodality treatment and patients feel healthy, but it is unlikely to return all pulmonary vessels back to normal. 


\section{Proposal for future research in CTEPH definition}

a) Both exercise-RHC as well as CPET still require establishment and validation of thresholds which could be incorporated into CTEPD definition

b) Research on definition of CTPED and CTEPH in patients with concomitant significant left heart and/ or lung disease

\section{Diagnosis}

In patients with $\mathrm{PH}$, the diagnosis of CTEPH is suspected when $V^{\prime} / Q^{\prime}$ scanning shows mismatched perfusion defects, and is confirmed by RHC and pulmonary vascular imaging [1, 19, 20]. Considering major advances in imaging technologies, the following aspects were discussed.

\subsection{Should single photon emission computed tomography replace planar $\mathrm{V}^{\prime} / \mathrm{Q}^{\prime}$ ?}

$V^{\prime} / Q^{\prime}$ scintigraphy is consistently acknowledged to be the most effective screening tool in the CTEPH diagnostic algorithm [1]. There is an emerging trend in clinical practice to replace planar $V^{\prime} / Q^{\prime}$ with single photon emission computed tomography (SPECT) $V^{\prime} / Q^{\prime}$. While most of the systematic reviews and meta-analyses professing the superiority of SPECT over planar imaging are based on acute PE [21-23], the higher sensitivity of $V^{\prime} / Q^{\prime}$ SPECT for CTEPH detection has also been demonstrated [24]. $V^{\prime} / Q^{\prime}$ SPECT imparts lower doses of isotopes per correct diagnosis [22]. The addition of low-dose computed tomography $(\mathrm{CT})$ to $V^{\prime} / Q^{\prime}$ SPECT improves specificity by identifying concomitant parenchymal lung disease [25]. SPECT quantitation can provide a measure of CTEPH disease severity [26]. Based on the review of the available literature [23-28] as well as clinical practice (supplementary table S4), most task force members use $V^{\prime} / Q^{\prime}$ SPECT as the methodology of choice where possible, with adherence to the simplified dichotomous interpretation criteria. The authors acknowledge that the transition from planar imaging to SPECT may not be easy, particularly for clinicians who may not be familiar with the three-dimensional anatomy. This could be overcome by generating two-dimensional planar images reprojected from SPECT data without losing diagnostic accuracy [27] (figure 1).

\subsection{Are dual-energy computed tomography or MR perfusion practical alternatives to $\mathrm{V}^{\prime} / \mathrm{Q}^{\prime}$ for CTEPH diagnosis?}

Over the past decade since its introduction, the role of dual-energy computed tomography (DECT) in $\mathrm{CTEPH}$ has evolved in its ability to detect parenchymal arterial perfusion and measure pulmonary vascular reserve. DECT iodine maps visualise parenchymal iodine distribution and are surrogate markers

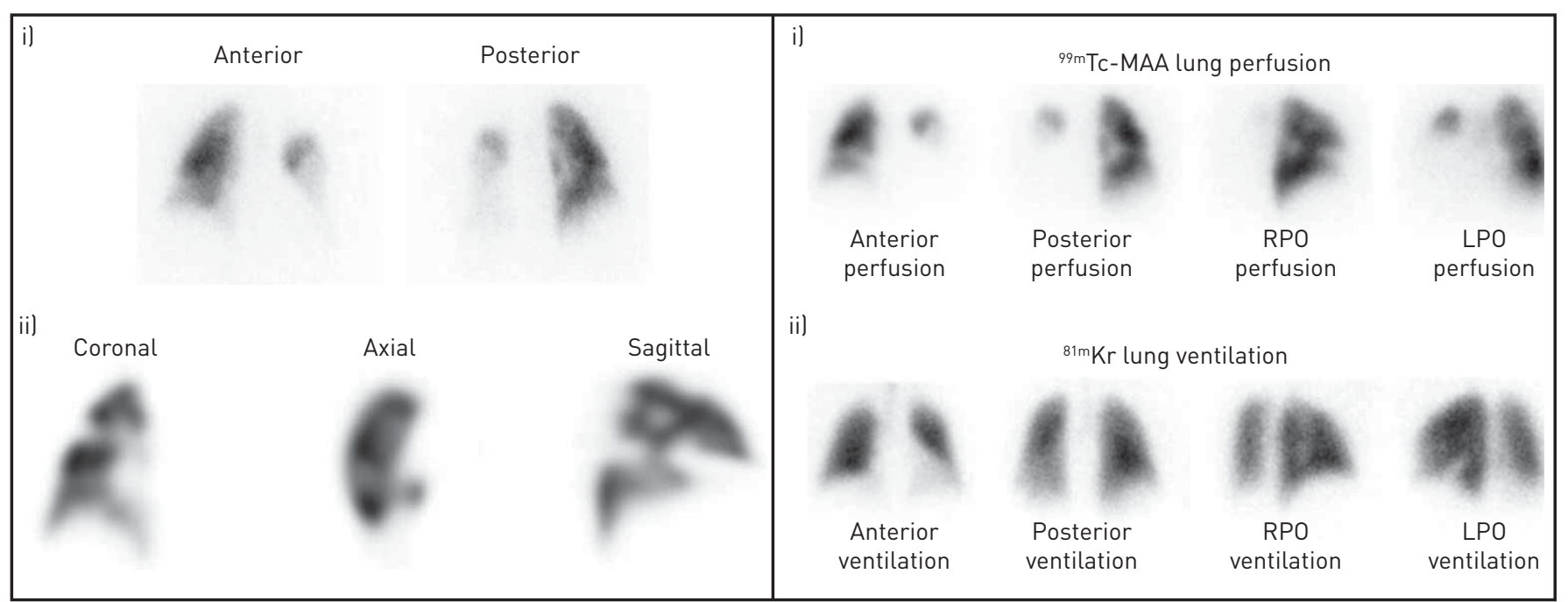

FIGURE 1 Ventilation/perfusion $\left(V^{\prime} / Q^{\prime}\right)$ scan. a) i) Anterior and posterior views from a planar $V^{\prime} / Q^{\prime}$ perfusion scan and ii) selected coronal, axial and sagittal perfusion images of the right lung from the corresponding single photon emission computed tomography (SPECT) show bilateral perfusion defects in a 60-year-old female with chronic thromboembolic pulmonary hypertension. Most of the left lung is nonperfused. The perfusion defects in the right lung are much better delineated on the SPECT series compared to the planar images. b) Simulated planar images

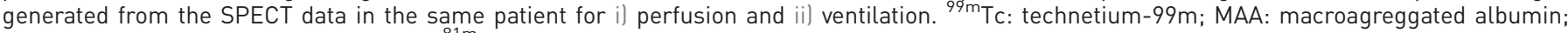
RPO: right posterior; LPO: left posterior; ${ }^{81 \mathrm{~m}} \mathrm{Kr}$ : krypton-81m. 
FIGURE 2 Dual-energy computed tomography (DECT). Axial view of a DECT image from a 50-year-old male with chronic thromboembolic pulmonary hypertension demonstrates eccentric thrombus (arrow) in the left main pulmonary artery extending into the lower lobe artery with corresponding perfusion defect in the left lower lobe (star). Also note the perfusion defect in the right lung (star).

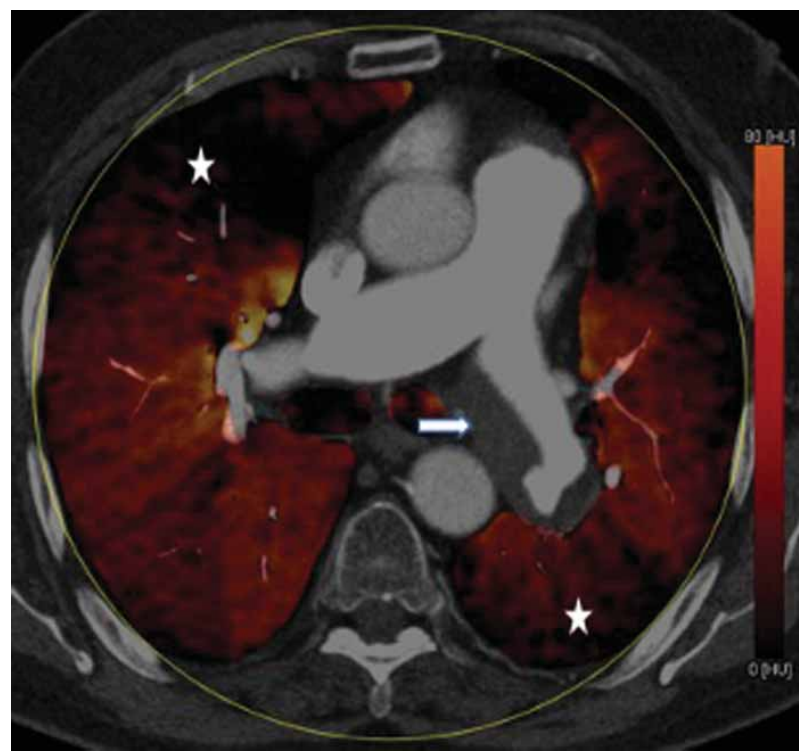

of lung perfusion [29]. DECT appearances in CTEPH is significantly different from pulmonary arterial hypertension (PAH) [30] (figure 2). DECT can be useful to differentiate acute PE and CTEPH based on the attenuation value of emboli [31]. In addition, detection of distal CTEPH is improved as perfusion defects are demonstrated even in the absence of visible morphological arterial abnormalities [32]. Automated quantification of pulmonary perfused blood volume (PBV) maps can provide objective measure of CTEPH severity [33,34]. There are several small volume studies comparing PBV maps to planar and $V^{\prime} / Q^{\prime}$ SPECT imaging with modest-to-good correlation [30, 35-38]. The lack of complete concordance is not surprising, as the two techniques are not physiologically equivalent.

The task force members acknowledge the advantages of DECT and its ability to provide anatomical and functional information for CTEPH in a single test, but do not currently replace $V^{\prime} / Q^{\prime}$ as a screening test for various reasons in their practice (supplementary table S4). The main limitation is the availability of CT systems capable of performing DECT and the attendant cost implications. In addition, there is a lack of standardised protocols for acquisition of PBV images that are consistent and reproducible. Further studies are required to overcome such limitations and validate the role of DECT in CTEPH.

Dynamic contrast enhanced (DCE)-MR perfusion is a three-dimensional breath-hold acquisition during first pass of gadolinium with a comparable sensitivity to planar and increased sensitivity to $V^{\prime} / Q^{\prime}$ SPECT for CTEPH detection $[39,40]$ (figure 3). A crucial factor limiting the use of DCE-MR as a screening tool is the lack of widespread availability of equipment as well as expertise. Other issues such as the technical
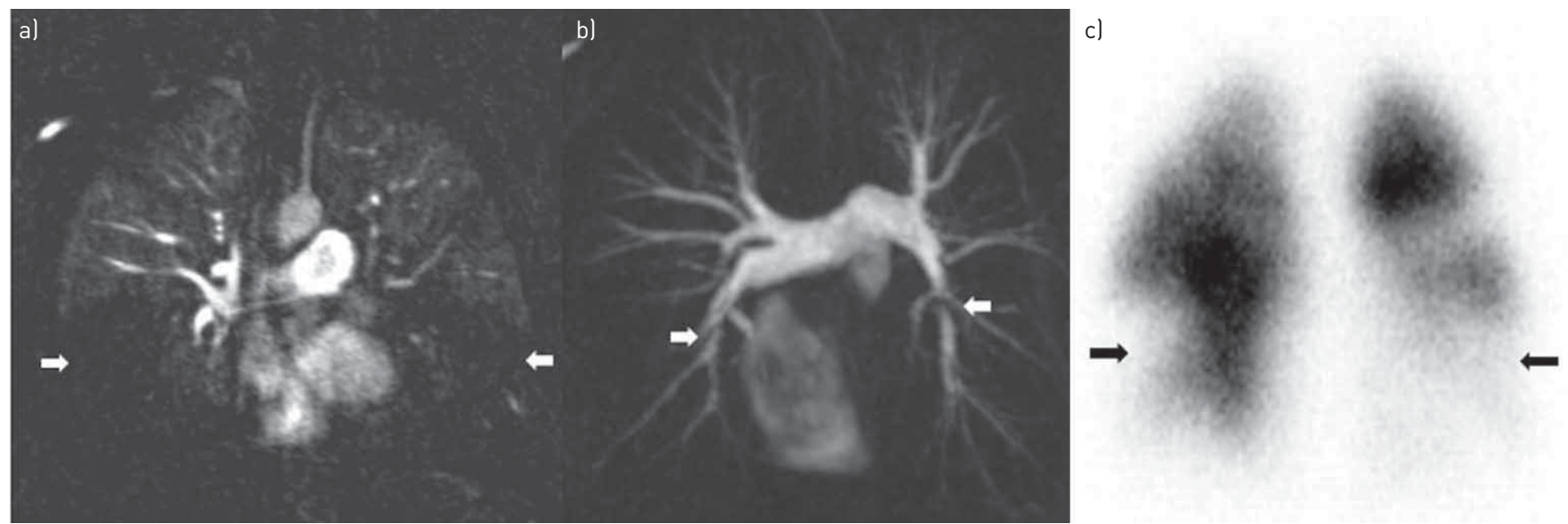

FIGURE 3 Magnetic resonance (MR) perfusion and angiography. a) 70-year-old male with chronic thromboembolic pulmonary hypertension with large bilateral perfusion defects in both lower lobes on the MR perfusion; b) the corresponding MR angiography demonstrates significant trifurcation webs in both lower lobes. c) There is good concordance with ventilation/perfusion scintigraphy (perfusion component, anterior view). 
demands and challenges involved in the evaluation of the lung parenchyma need to be surmounted before routine implementation of $\mathrm{MR}$ as a single imaging test in CTEPH.

\subsection{Is high-quality CTPA adequate for diagnosis or is there a need for digital subtraction angiography?}

Ongoing technological advances continue to refine the role of CT in the CTEPH diagnostic algorithm. Modern CTPA may be noninferior to $V^{\prime} / Q^{\prime}$ in diagnosing CTEPH [41], but a fundamental reservation is that a negative CTPA cannot exclude CTEPH, as small vessel disease can be missed. CTPA technical quality is a significant contributor to the variation in its usage, as is the inconsistency in CT interpretation in non-CTEPH expert centres [42]. A recent meta-analysis demonstrated that high-quality CT studies had a pooled sensitivity of $99 \%$ and specificity of $97 \%$ for CTEPH detection on a vessel-based analysis [43]. Some high-volume CTEPH expert centres use CT data to provide a roadmap of the location and extent of disease for operability assessment.

Most task force members use a high-quality CTPA (supplementary table S5) as a good noninvasive substitute for diagnosis of proximal CTEPH. Additionally, experienced operators use CTPA for evaluation of suitability for PEA in selected cases where the disease distribution is proximal.

\subsection{Role of new CT advances such as cone beam computed tomography and area detector computed tomography}

Digital flat-panel detector cone beam computed tomography (CBCT) is a recent innovation that can be used in conjunction with $\mathrm{C}$-arm systems to provide projection radiography, fluoroscopy, digital subtraction angiography (DSA) and volumetric CT. CBCT has the ability to provide better delineation of distal lesions in CTEPH (figure 4). In comparison to DSA and conventional CT, CBCT is more sensitive in depicting subsegmental arterial abnormalities, and more accurate in detection and characterisation of these lesions compared to selective angiography [44-47]. CBCT is a useful tool for BPA procedure guidance, as it helps to obtain appropriate working projections and allows for targeted selective imaging and treatment. BPA guided by CBCT $[48,49]$ or ECG-gated and area detector computed tomography (ADCT) (320-row) [50] is effective and remarkably safe. These advantages must be balanced against the invasive nature of CBCT. There is limited literature on the radiation dosimetry of CBCT in CTEPH patients, but it is clear that the dose will vary depending on the protocol used and is on average $\sim 3 \mathrm{mSv}$ [44].

In summary, these are high-end techniques that provide better anatomical definition for CTEPH diagnosis and treatment, but are expensive and are not widely available. As they are still in evolution, there is no standardisation of the protocol for their usage. The benefits of the technology require validation with prospective trials before recommendation for routine clinical use.
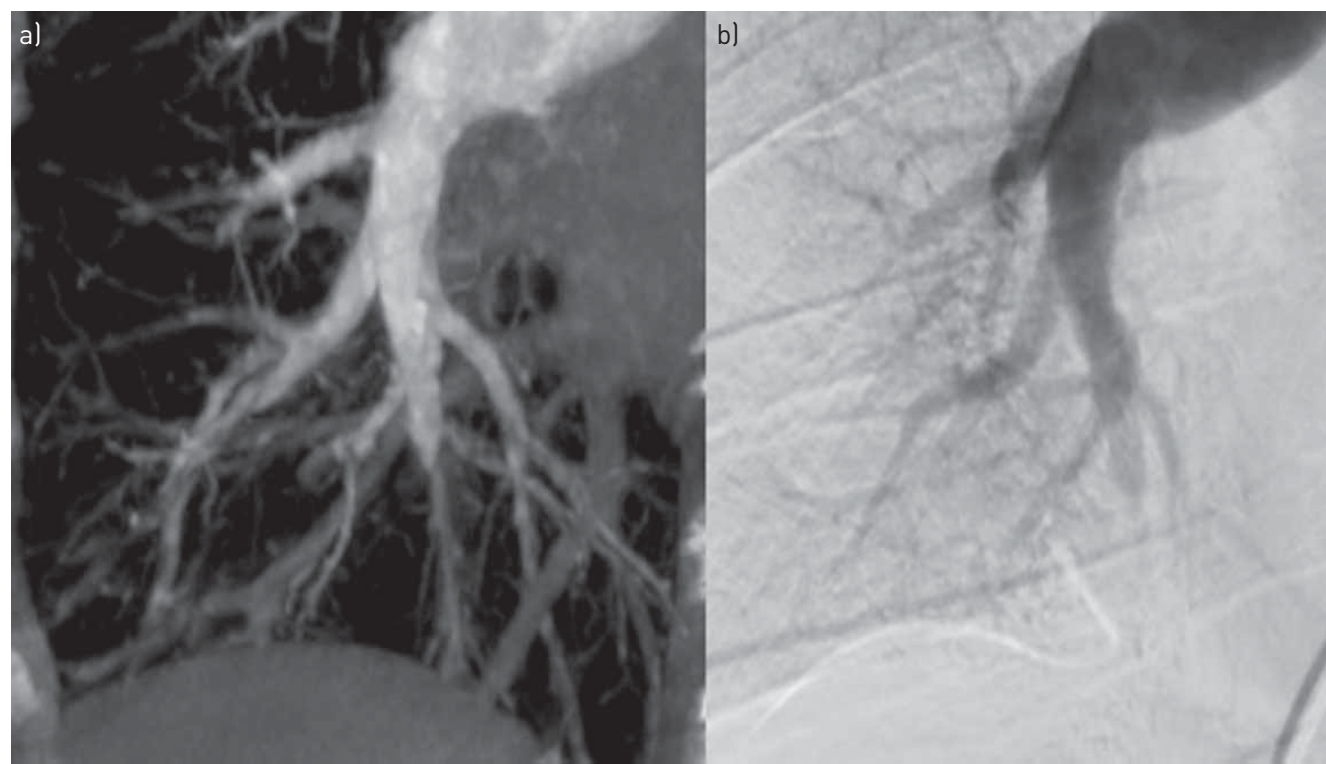

FIGURE 4 Cone beam computed tomography (CBCT). Selected images from a) CBCT and b) corresponding conventional catheter pulmonary angiography in a 45-year-old male with chronic thromboembolic pulmonary hypertension to illustrate the superior depiction of the pulmonary arterial abnormalities at the segmental and the subsegmental level on the CBCT. 
2.5 What is the utility of cardiac MRI in the diagnostic algorithm?

Although well suited for CTEPH diagnosis and follow-up, MRI is not yet fully integrated into the CTEPH diagnostic algorithm. Its use is heavily dependent on local practice with routine implementation only in a few high-volume institutions where there is clustering of expertise.

MR angiography for diagnosis

A comprehensive stepwise protocol of MR perfusion followed by high spatial resolution MR angiography improves the diagnostic performance of MR. It is essential to review the source images and use multiplanar reformations to evaluate the complex vascular anatomy. MR angiography maximum intensity projections (MIP) provide an overview of the pulmonary arterial tree in a format that is comparable to DSA.

Perfusion MRI has sensitivity of $97 \%$, specificity $92 \%$, positive predictive value $95 \%$ and negative predictive value $96 \%$ for detecting CTEPH, a performance comparable to $V^{\prime} / Q^{\prime}$ scintigraphy and CTPA [40]. In a study comparing the efficacy of contrast-enhanced MR pulmonary angiography (CE-MRA), CTPA and DSA, CE-MRA demonstrated a sensitivity and specificity of $83.1 \%$ and $98.6 \%$ at the main/lobar level and $87.7 \%$ and $98.1 \%$ at segmental level for diagnosing CTEPH, but was outperformed by DSA for depiction of subsegmental arteries [51]. Besides, CE-MRA was demonstrated to identify proximal and distal CTEPH with a high sensitivity and specificity of $98 \%$ and $94 \%$, respectively [52].

\section{Right ventricular function in evaluating PH severity at the time of diagnosis}

Cardiac MR is the reference standard for the assessment of right ventricle (RV) size and systolic function [53] and is well equipped to consistently provide accurate evaluation of RV function in the diagnostic work-up and follow-up of patients with CTEPH. MRI has been used to noninvasively demonstrate the technical success of PEA as evidenced by the RV reverse remodelling and improvement in the interventricular septal dyssynchrony and pulmonary clearance [54-56]. BPA-induced RV reverse remodelling has also been demonstrated using cardiac MR in patients with inoperable disease [57]. Late gadolinium enhancement (LGE) at the RV insertion points is strongly associated with elevated PAP, but its prevalence in CTEPH is not clearly defined, and its prognostic significance remains uncertain. In a subset of 39 patients with CTEPH from the ASPIRE registry [52], 97\% had RV hinge point enhancement, leading the authors to conclude that its presence is highly sensitive for the detection of severe increases in RV afterload. Parametric mapping techniques have shown that native T1 values in the septum are elevated in CTEPH even in the absence of LGE [58]. Larger trials are needed to understand the potential of quantification of septal fibrosis as a prognostication and therapy-monitoring tool in the future.

Is noninvasive (MR-based metrics) measurement of haemodynamic parameters prime time? i.e. can we replace $R H C$ with MR-based data?

Attempts to correlate MR-based haemodynamic parameters with RHC data have thus far met with limited success [52, 59-64]. Various multiparametric models have been introduced to estimate mPAP and PVR from cardiac MR data, but validation is challenged by the small number of comparable studies and paucity of prospective clinical cohorts. However, it is likely that advances such as strain imaging, parametric mapping and four-dimensional flow MR hold great potential in providing key metrics for CTEPH screening and monitoring.

MRI has the potential to offer a noninvasive holistic approach to CTEPH diagnosis and follow-up, but there is widespread lack of acceptance in the clinical community due to perceived as well as genuine issues regarding access to the technique, bias about protracted acquisition times and lack of expertise in interpretation. Multicentre prospective trials and focused training are required to make a paradigm shift before MR can be routinely integrated into the CTEPH diagnostic algorithm.

\subsection{Diagnostic algorithm}

Summarising recent data, the diagnostic algorithm for CTEPH used by task force members in their practice is shown in figure 5 .

\section{Statements: diagnosis}

2.1 $V^{\prime} / Q^{\prime}$ scintigraphy remains the most effective screening tool to exclude CTEPD. $V^{\prime} / Q^{\prime}$ SPECT has been shown to be superior to planar imaging and is the methodology of choice. Because the transition from planar imaging to SPECT may not be easy for clinicians unfamiliar with the three-dimensional anatomy, two-dimensional planar images can be reprojected from SPECT without losing diagnostic accuracy.

2.2 Alternative perfusion techniques such as dual energy computed tomography and magnetic resonance perfusion have numerous theoretical advantages over $V^{\prime} / Q^{\prime}$, but are more technically challenging and 


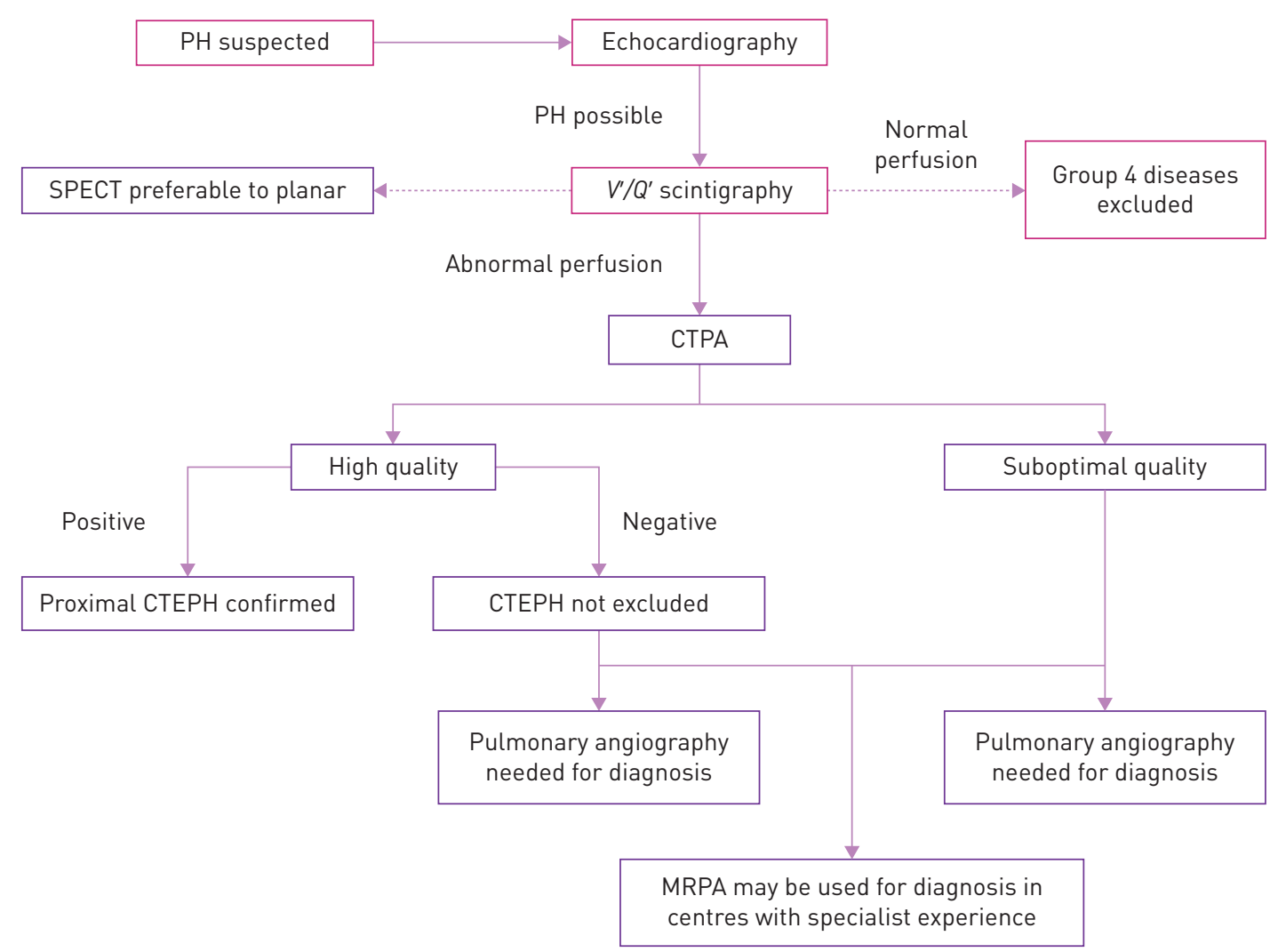

FIGURE 5 Chronic thromboembolic pulmonary hypertension (CTEPH) diagnostic algorithm used by task force members. The figure describes the current practice of how the members of the task force diagnose CTEPH and is not intended as a general recommendation. $\mathrm{PH}$ : pulmonary hypertension; SPECT: single photon emission computed tomography; $V^{\prime} / Q^{\prime}$ : ventilation/perfusion ratio; CTPA: computed tomography pulmonary angiography; MRPA: magnetic resonance pulmonary angiography.

expensive, with limited availability and lacking multicentre validation. Hence, they are not replacing $V^{\prime} / Q^{\prime}$ in current clinical practice.

2.3 High-quality CTPA is adequate for diagnosis of proximal CTEPH, but a negative CTPA, even if high-quality, does not exclude CTEPH, as distal disease can be missed.

2.4 Cone beam computed tomography and area detector computed tomography allow for more accurate visualisation of subsegmental vasculature and have been shown to be useful for procedural guidance for BPA. The benefits of the technology require validation in prospective trials before recommendation for routine clinical use.

2.5 MRI is not yet fully integrated into the CTEPH diagnostic algorithm, because its use is heavily dependent on local practice and is routinely implemented only in a few high-volume institutions where there is clustering of expertise.

Proposal for future research in CTEPH diagnosis

a) Prospective multicentre validation of DECT for screening and diagnosis of CTEPH

b) Prospective multicentre validation of MR (perfusion, angiography and parametric models) for CTEPH screening, diagnosis and evaluation of disease severity

\section{Epidemiology}

3.1 Incidence and prevalence of CTEPH: is CTEPH a complication of acute PE, or is CTEPH often misclassified as PE?

The prevalence of CTEPH in the general population is difficult to evaluate, since the disease is rare and may be underdiagnosed. Some information can be derived from national $\mathrm{PH}$ registries in which CTEPH represents $\sim 20 \%$ of patients referred to main $\mathrm{PH}$ centres [65]. Estimated CTEPH prevalence from registries ranges from 3.2 per million in the Spanish registry to 38 per million in the UK National Audit of PH $[66,67]$. An epidemiological analysis of the burden of CTEPH in different countries estimated its prevalence ranging from 19 per million in Japan to 30-50 per million in the United States and Europe 
[68]. A recent study estimated the prevalence of CTEPH based on a case ascertainment algorithm within the French exhaustive hospital discharge database (PMSI) [69]. Based on 3138 patients hospitalised for CTEPH in 2015 in France, and assuming that patients were hospitalised at least once a year, the prevalence of CTEPH was estimated 47 cases per million (range 43-50 cases per million).

CTEPH is usually considered as a complication of acute PE, with $50-75 \%$ of patients with CTEPH having a documented history of acute PE [70-72]. In Japanese patients, only 15\% have a history of acute PE [73], supporting the hypothesis of a different phenotype of CTEPH in the Japanese population, together with the observed female preponderance, a lower proportion of coagulopathies [72] and less proximal thrombus and fresh red thrombus components in PEA specimens [74].

The cumulative incidence of CTEPH after acute PE is not exactly known and is reported as ranging from $0.1 \%$ to $11.8 \%$ in the first 2 years after symptomatic PE [75-79]. This wide range could be explained by referral bias, the paucity of early symptoms and the difficulty of differentiating acute PE symptoms from pre-existing CTEPH [77-79]. A systematic review and meta-analysis of studies including consecutive PE patients followed for CTEPH (16 studies including 4047 PE patients who were followed-up for $>2$ years) showed a CTEPH incidence of $0.56 \%$ (95\% CI $0.1-1.0 \%)$ in all comers. In survivors of PE and in survivors without major comorbidities, incidence of CTEPH was 3.2\% (95\% CI 2.0-4.4\%), and 2.8\% (95\% CI 1.5 $4.1 \%$ ), respectively [79]. The authors suggested that the $\sim 3 \%$ incidences in the survivor categories may be more relevant for daily clinical practice. They emphasised that studies that assessed CTEPH diagnosis by tests other than RHC provided an overestimated incidence of CTEPH, underscoring the need of an accurate workup in patients who remain symptomatic after an acute PE. However, in a more recent multicentre observational screening survey for the detection of CTEPH following PE (screening performed at 6,12 and 24 months using a stepwise algorithm including dyspnoea phone-based survey, echocardiography, RHC and radiological confirmation of CTEPH), the CTEPH incidence was estimated as 3.7 per 1000 patient-years with a 2-year cumulative incidence of $0.79 \%$ [80].

Finally, there are very strong arguments for frequent misclassification of CTEPH as PE [75, 77, 79]. In a prospective multicentre study conducted in three centres in France, 146 patients with an acute PE were followed-up for a median 26 months. Among the seven patients diagnosed with CTEPH (incidence 4.8\%, 95\% CI 2.3-9.6\%), echocardiography and CT performed at the time of the acute PE suggest that a majority of them had previously unknown $\mathrm{PH}$, indicating that a first clinical presentation of CTEPH may mimic acute PE [77].

\subsection{How many patients with persistent perfusion defects after acute PE have CTEPD?}

According to $V^{\prime} / Q^{\prime}$ scan studies, up to $50 \%$ of patients have persistent perfusion defects after an acute PE [81-83], with discrepant clinical relevance. Some studies reported an impact of residual obstruction on pulmonary haemodynamics, functional class, exercise capacity and outcome $[83,84]$, while the more recent ELOPE study showed no correlation with exercise capacity [85]. Some of these "persistent perfusion defects" could also be pre-existent to the acute PE, precluding any clear conclusion. Considering the "pyramid" of complications [65], CTEPD without PH probably occurs more often than CTEPH. So, if the prevalence of CTEPH after PE in survivors is $2-3 \%$ [79], the prevalence of CTEPD is probably higher than that.

\subsection{Is CTEPD without PH an early stage of CTEPH?}

There is no definitive answer to that question. Our limited knowledge of the natural history of CTEPH is based on historical data prior to the current treatment era. Patients with mild $\mathrm{PH}(\mathrm{mPAP}<30 \mathrm{mmHg}$ ) and "borderline PH" (mPAP 21-24 mmHg) were thought to have a good prognosis, with low probability of progressing to severe $\mathrm{PH}$ when treated solely with anticoagulation $[86,87]$, although these studies lack information on the severity of the thrombotic burden. According to clinical experience, some patients remain stable while others experience a rapid unexplained deterioration. When focusing on CTEPD patients without $\mathrm{PH}$ but extensive thromboembolic disease who underwent surgery, PEA specimens were no different from CTEPH patients [2].

\subsection{Could CTEPD incidence be different in patients treated with vitamin $\mathrm{K}$ antagonists and non-vitamin $\mathrm{K}$ antagonist oral anticoagulants? Could it be different in patients treated with different NOACs? Could the incidence be influenced by dosing strategy in NOACs?}

Current literature does not suggest any difference in CTEPD incidence in patients with venous thromoboembolism (VTE) treated with vitamin $\mathrm{K}$ antagonist (VKAs) and non-vitamin $\mathrm{K}$ antagonist oral anticoagulants (NOACs). Previous studies have shown that persistent perfusion defects occur despite adequate anticoagulation (mostly with VKA; in up to 50\% of patients) [81-83]. In the literature on thrombus resolution in deep vein thrombosis, some industry-sponsored studies have suggested that 
rivaroxaban may lead to better venous patency than VKA $[88,89]$; however, more recent independent studies did not confirm this $[90,91]$. The fact that the risk of early recurrent VTE is no different between NOAC- versus VKA-treated patients in randomised controlled trials (RCTs) is a strong argument to say that the choice of anticoagulant drug should not influence the risk of CTEPH. By preventing recurrent VTE, due to more patients getting lifelong treatment, it may lower the prevalence of CTEPH (half- or full-dose NOAC has same efficacy in reducing the risk of recurrent VTE).

However, in some specific situations, treatment with NOACs (which is recommended by current ESC guidelines for diagnosis and treatment of acute PE in preference to VKA, due to their ease of use and better tolerability $[92,93]$ ) could be associated with higher risk of recurrent thromboembolic events than VKA therapy (recurrent PE is a risk factor associated with CTEPH [65]). It was confirmed in patients with high-risk antiphospholipid syndrome (lupus anticoagulant, anticardiolipin antibodies, and anti- $\beta_{2}$-glycoprotein I antibodies) [94]. In other specific clinical situations (e.g. obesity with a body mass index $>40 \mathrm{~kg} \cdot \mathrm{m}^{-2}$ or a body weight $>120 \mathrm{~kg}$, concomitant medication with proton pump inhibitors or $\mathrm{H}_{2}$ antagonists which can reduce gastrointestinal absorption of dabigatran) pharmacokinetic aspects of NOACs have to be carefully taken into account to optimise the risk-benefit profile of these drugs in prevention of recurrent thromboembolic events and perhaps CTEPD [95, 96].

\subsection{Should the incidence and prevalence of CTEPD be analysed in specific subpopulations? Could} this information be useful for the screening recommendation?

Clinical conditions such as permanent intravascular devices (pacemaker, infusion chamber, ventroarticular shunts), inflammatory bowel diseases and essential thrombocythaemia have been identified as risk factors for CTEPH [97, 98]. Prevalence of CTEPH in this population of patients is not sufficiently described [68], precluding recommendations for screening in nonsymptomatic patients.

\section{Statements: epidemiology}

3.1 CTEPH is a rare and underdiagnosed complication of PE and, upon first presentation, may be misclassified as acute PE.

3.2 Persistent perfusion defects after acute PE are common, but have highly variable clinical relevance, ranging from completely asymptomatic to established CTEPH.

3.3 We have no evidence to suggest that CTEPD without PH is an early stage of CTEPH, because of a lack of data on its natural history.

3.4 Current literature could not demonstrate any difference in CTEPH incidence in patients with venous thromoboembolism treated with VKAs and NOACs, but rigorous studies have not been done.

3.5 Screening for CTEPH in asymptomatic patients with specific risk factors could be effective, but is not supported by any data.

\section{Follow-up after acute PE}

\subsection{Is earlier detection of CTEPH relevant for outcome?}

In daily practice, it may take 14-24 months from symptom onset for CTEPH to be diagnosed [71, 99101]. Although this diagnostic delay was not associated with operability in the European CTEPH registry, longer delay was shown to correlate to higher systolic pulmonary arterial pressure (SPAP) at diagnosis and increased risk of death $[71,99,102]$. Even in the relatively short time elapsed between diagnosis of CTEPH and referral to a surgical centre (mean 4.4 months), it was observed that cardiac output decreased and mPAP increased slightly [103]. In addition, progressive increases of PAP and PVR were observed 115 years after diagnosis in patients with initial mPAP $>30 \mathrm{mmHg}$ [86]. This can be attributed to the natural course of CTEPH involving the development of a secondary microvasculopathy, further discussed in section 5 , and confirms that a timely diagnosis of CTEPH is important.

\subsection{How can a patient at high risk of CTEPH after acute PE be identified, and how can we better} identify patients who already have CTEPH while presenting with what appears to be acute PE?

CTEPH is usually considered at two time points: 1) when presenting with acute PE, if radiological signs suggest CTEPH on the CTPA performed to diagnose PE (figures 6 and 7 and supplementary table S6), and/or if estimated sPAP $>60 \mathrm{mmHg}$ [77, 104-107]; and 2) more classically, when dyspnoea or functional limitations persist in the clinical course of PE [108-111]. A third condition could concern asymptomatic patients with risk factors for CTEPH or a high pre-test probability [112] (supplementary table S7).

Clearly, the presence of CTEPH characteristics on a CTPA performed in the setting of acute PE prompts the suspicion of CTEPH as primary diagnosis. CTPA signs of CTEPH include eccentric wall-adherent filling defect, abrupt tapering and truncation, complete occlusion and pouch defects, intimal irregularity, intravascular webs and bands, stenosis, post-stenotic dilatation and vascular tortuosity [93]. It has been 

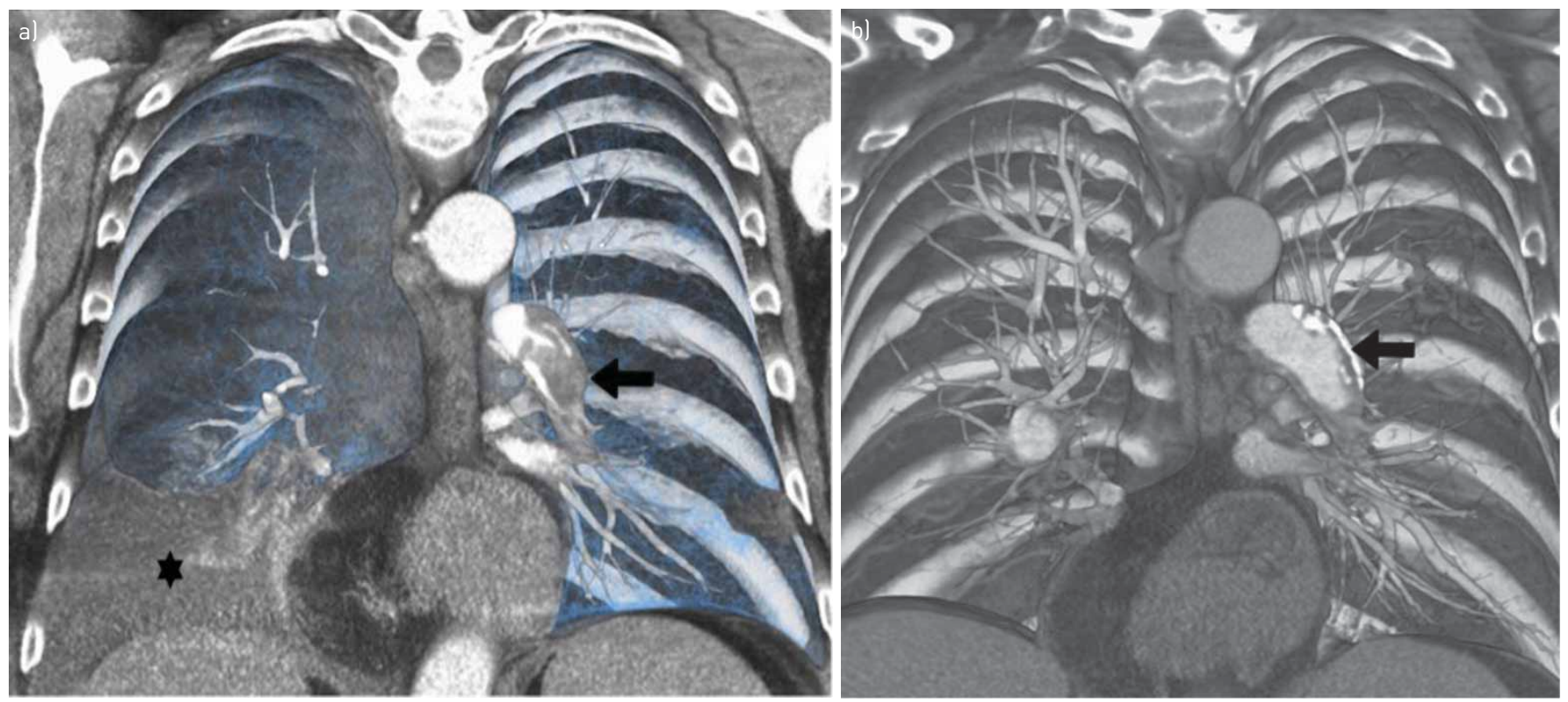

FIGURE 6 a) Coronal volume rendered computed tomography pulmonary angiography images demonstrate acute pulmonary embolism in the left lower lobe (arrow; star indicates right pleural effusion); b) 2 years later, this has evolved into chronic thromboembolic pulmonary hypertension with eccentric calcific clot (arrow).

shown that most patients with CTEPH after PE had several signs of CTEPH on the index CTPA, indicating acute-on-chronic events rather than acute PE $[77,106]$. If evaluated by experts, the presence of three or more radiological characteristics of CTEPH on CTPA for suspected PE was virtually diagnostic (specificity 96\%), although less-experienced radiologists may be less likely to recognise this $[42,106]$.

Notably, CTPA protocols are largely dependent on the available hardware and vary according to patient age, size, motion and cardiac function. Their fundamental goal is to achieve adequate pulmonary arterial enhancement such that thrombus can be distinguished from intraluminal contrast medium. The theoretic minimum intraluminal attenuation of blood required to visualise acute and chronic pulmonary
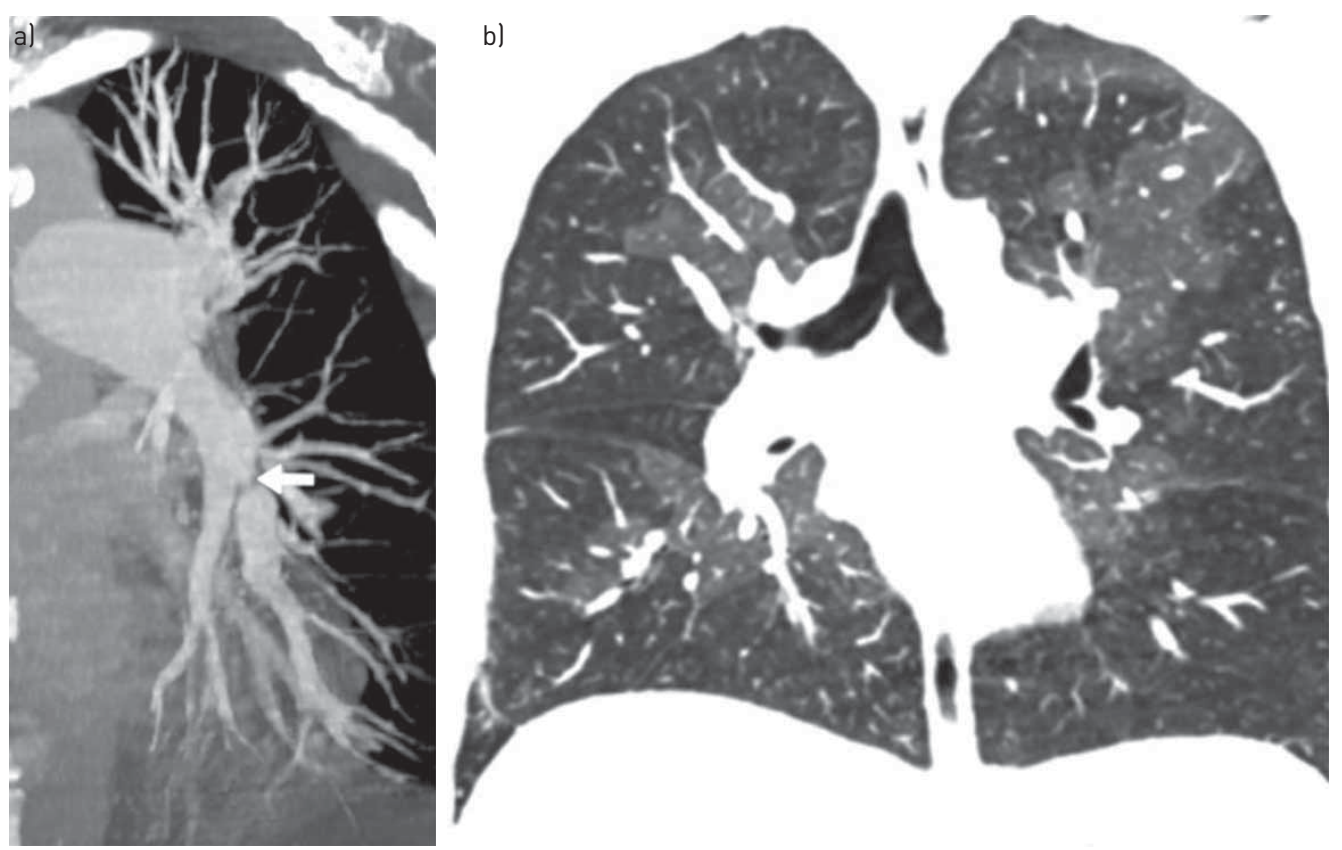

FIGURE 7 a) Coronal computed tomography pulmonary angiography (CTPA) reconstruction to show segmental stenosis and post-stenotic dilatation in left lower lobe in a chronic thromboembolic pulmonary hypertension case (arrow); b) coronal CTPA lung window demonstrates mosaic attenuation. 
thromboemboli are 93 and $211 \mathrm{HU}$, respectively [113]. CTPA performed for diagnosing acute PE does not involve ECG gating. Considering this, and especially in the acute setting of haemodynamic and respiratory compromise, standard CTPA PE protocols may therefore be less optimal for recognising signs of CTEPH than dedicated protocols for diagnosing CTEPH. Development and validation of artificial intelligence based software to detect CTEPH on standard CTPA images could help improve diagnostic accuracy in clinical practice.

\subsection{Which PE patients should be systematically assessed for the presence of CTEPH and by which primary diagnostic test?}

The ESC/ERS guidelines recommend against routine echocardiography in all PE survivors because of the low yield and to prevent overdiagnosis [93]. Similarly, the Canadian Thoracic Society (CTS), after a formal clinical practice guideline development process, involving systematic literature searches and evaluation of the evidence according to a Grading of Recommendations, Assessment, Development and Evaluations approach, recommended against routine screening after acute PE [114]. In patients with persistent or new-onset dyspnoea after PE, the diagnostic work-up starts with echocardiography, followed by $V^{\prime} / Q^{\prime}$ scintigraphy and RHC with pulmonary angiography [1], as discussed in section 2 .

Alternative tests may also provide diagnostic clues to CTEPH or leave this diagnosis very unlikely (supplementary table S8). They could be applied to patients with a low CTEPH prevalence, i.e. to rule out $\mathrm{CTEPH}$ rather than to confirm the disease, and/or establish an alternative diagnosis for the clinical presentation, since the majority of post-PE functional limitations will be caused by deconditioning [12, 111, 115-117]. CPET, $V^{\prime} / Q^{\prime}$ scanning or the combination of ECG and N-terminal pro-brain natriuretic peptide (NT-proBNP) in the Leiden CTEPH rule-out criteria may be useful in this setting [112].

Concerning asymptomatic post-PE patients with risk factors for CTEPH (supplementary table S7), common sense needs to be used in the absence of evidence.

\subsection{What is the optimal timing of a diagnostic strategy for early CTEPH detection after PE?}

The optimal timing of diagnostic tests for CTEPH may be 3-6 months after the acute PE, coinciding with the moment of routine evaluation of the duration and intensity of anticoagulant treatment [93]. This timeframe can also be used to evaluate whether patients tolerate anticoagulant treatment, which is essential after PEA to prevent recurrent pulmonary emboli. Of course, patients with signs of severe PH or overt right heart failure need to be referred earlier to a CTEPH expertise centre to determine the need for immediate treatment. Improving education of PE caretakers, higher awareness for CTEPH and validation of clinical/radiological algorithms for earlier CTEPH diagnosis will probably help ensure earlier referral and improved prognosis.

\section{Statements: follow-up after acute PE}

4.1 Earlier diagnosis of CTEPH may be relevant for patient outcomes. It can be achieved by targeting patients with acute PE with risk factors for CTEPH or with radiological findings suggesting CTEPH.

4.2 Patients at risk of CTEPH can be identified by accurate assessment of the CTPA images used to diagnose PE, individual risk factors for $\mathrm{CTEPH}$, and symptoms of functional limitations and/or right heart failure in the course of PE.

4.3 Echocardiography is the test of choice in patients with suspected CTEPH. Other tests such as Leiden CTEPH rule-out criteria, $V^{\prime} / Q^{\prime}$ scan or CPET could be used to exclude the presence of CTEPH and/ or to establish an alternative diagnosis.

4.4 The optimal timing for considering CTEPH in patients with PE is at the routine 3-month follow-up visit, but earlier work-up may be necessary in highly symptomatic or deteriorating patients.

\section{Recommendations for future research and training}

a) Prospective validation of algorithms for early diagnosis of CTEPH after PE

b) Dedicated training of radiologists to increase awareness for, and skills to detect, CTEPH on routine CTPA scans performed in the setting of suspected acute PE

c) Development and validation of artificial intelligence-based software to detect CTEPH on standard CTPA images, to improve diagnostic accuracy of dedicated CTPA reading for signs of CTEPH in clinical practice

\section{Pathophysiology}

\subsection{Vascular lesion characteristics and mechanisms of increased resistance in CTEPH}

Anatomically, two different vascular lesions participate in the increase of PVR in CTEPH patients: obstruction of pulmonary arteries by unresolved organised fibrotic clots and a secondary microvasculopathy (figure 8). 


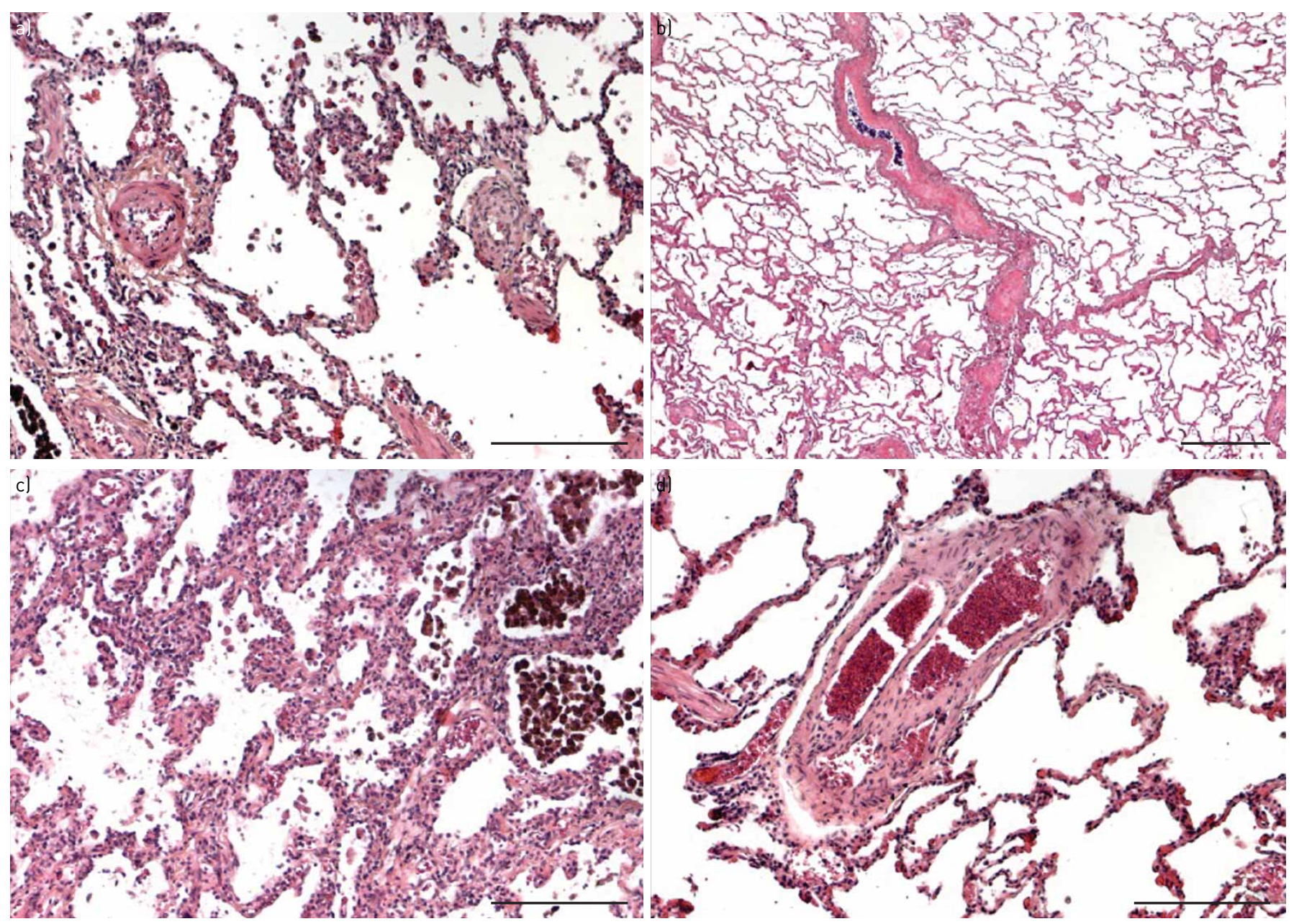

FIGURE 8 Pulmonary vascular lesions from explanted lungs of patients suffering from chronic thromboembolic pulmonary hypertension. a) Proliferative, fibromuscular lesions of microvessels (centre left and centre right, arterioles/venules) of $<100 \mu \mathrm{m}$ in diameter; note the concentric wall thickening. Scale bar $200 \mu \mathrm{m}$. b) Intimal fibrosis and partial occlusion of a septal vein and pre-septal venules in its vicinity. Scale bar $500 \mu \mathrm{m}$. c) Capillary proliferation within the alveolar septa, reminiscent of pulmonary capillary hemangiomatosis; note the numerous packed haemosiderin-laden macrophages within the alveoli (top right). Scale bar $200 \mu \mathrm{m}$. d) Colander-like lesion in a small pulmonary artery, corresponding to a partially recanalised thromboembolic lesion. Scale bar $200 \mu \mathrm{m}$.

Obstruction of the pulmonary arteries by unresolved fibrotic clots

Major vessel pulmonary artery obstructions are partially recanalised, collagen-rich, wall-adherent clots within the elastic type main pulmonary arteries, reaching down to distal pulmonary arteries at the intra-acinar level (figure 8a,d). Proximally, these lesions appear to be the histological counterpart to what is known as slits, webs, stenoses or pouching on vascular imaging. In addition, large, elastic-type pulmonary arteries that are subject to longstanding increased PAP lead to atheromatous lesions with increased wall stiffness [118], resembling aortic wall changes in patients with systemic hypertension. Distally, typical obstructive chronic thromboembolic lesions appear as occluded vessels with recanalisation and occasionally multiple secondary lumina called colander-like lesions [119, 120].

\section{Secondary microvasculopathy}

Secondary microvasculopathy was first described by MOSER and BLOOR [121] in human lung tissue obtained by biopsy or at autopsy. These lesions involve the wall of muscular pulmonary arteries (50$500 \mu \mathrm{m}$ in diameter) and resemble PAH arteriopathy. In human disease [121, 122] as well as in animal models $[17,123]$, these PAH-like lesions predominate in lung regions nonobstructed by proximal clots (figure $8 \mathrm{a}$ ). The proposed physiopathological mechanism is that, in the presence of an obstruction of proximal pulmonary arteries, the pulmonary blood flow is redistributed to nonobstructed lung areas leading to high flow and pressure, as well as increased endothelial shear stress [121]. However, MosER in his initial report recognised that this hypothesis does not explain a microvasculopathy that can also be observed in lung areas downstream to completely or partially obstructed proximal vessels. 
The microvasculopathy is characterised by remodelling at the level of pre-capillary arteries, capillaries (pulmonary haemangiomatosis-like areas in severe cases) and pulmonary venules (pulmonary veno-occlusive-like). These venous microvascular changes have been observed in lung territories distal to completely or partially obstructed proximal pulmonary arteries, both in humans and in animal models $[17,18,121,122]$ (figure $8 \mathrm{a}-\mathrm{c}$ ). A possible physiopathological mechanism is the development and hypertrophy of systemic bronchial arteries and anastomoses between bronchial arteries and the pulmonary circulation downstream of pulmonary artery stenoses and occlusions [124] (figure 9). In CTEPH patients and in chronic experimental obstruction of the pulmonary arteries in animals, the systemic bronchial arteries are significantly enlarged anatomically and are proliferating (bronchial artery arterio-/angiogenesis [125]). In addition, substantial bronchopulmonary anastomoses on the pre- and the post-capillary side lead to transmission of systemic blood pressure to the pulmonary circulation [17, 18]. In addition, bronchopulmonary shunting can be observed within the core of the organising clot, where proliferating vasa vasorum (being the equivalent to bronchial arteries) drain blood into bronchial veins, which, in turn, feed into the pulmonary veins.

In a piglet model [126], ligation of the left main pulmonary artery associated with progressive embolisation of the right lower lobe artery leads to hypertrophy of the systemic lung vasculature locally,
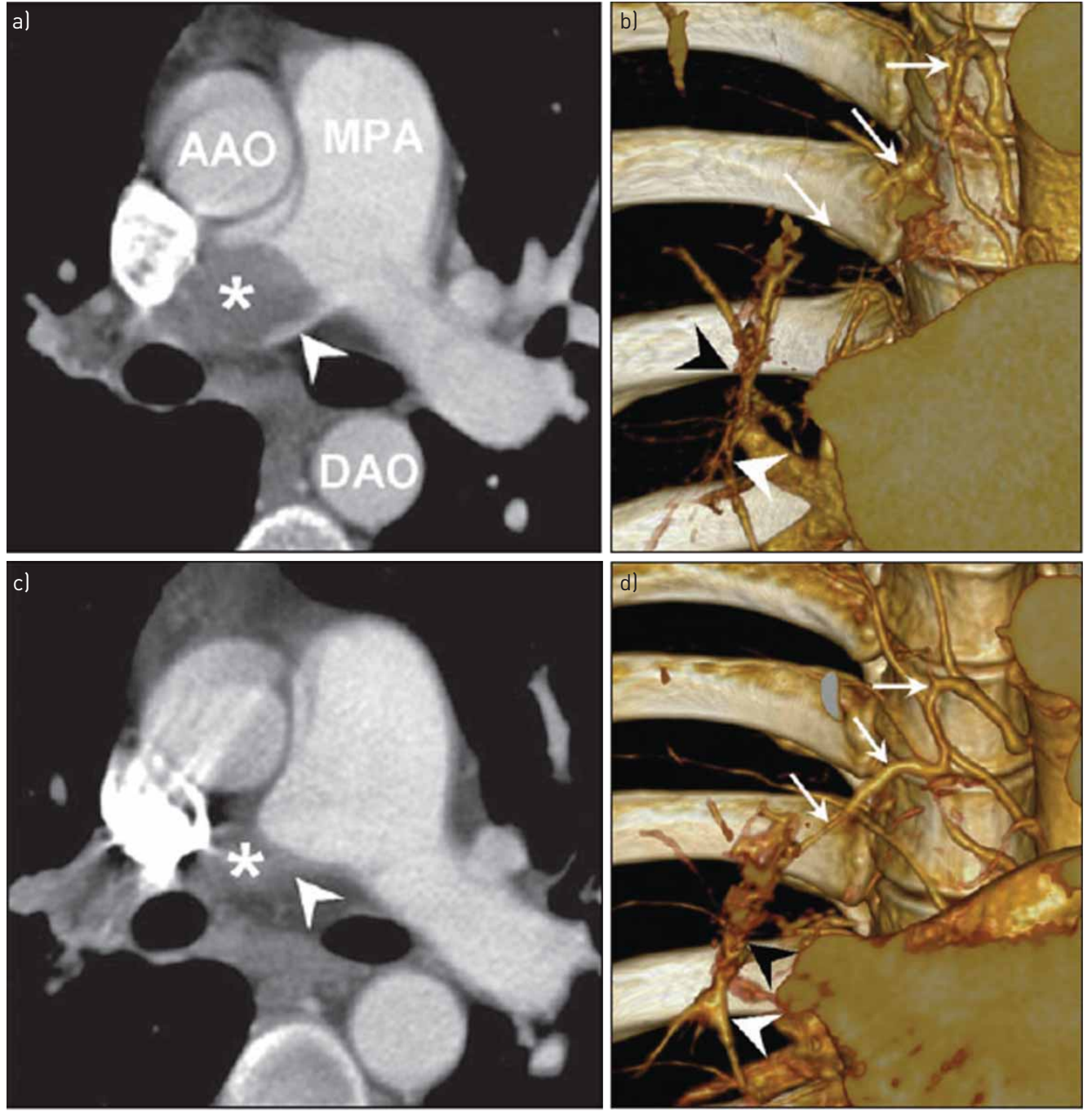

FIGURE 9 a) Computed tomography pulmonary angiography (CTPA) shows complete obstruction of right central pulmonary artery due to acute emboli (asterisk); b) slab volume-rendering image shows normal size of bronchial artery (arrows). Distal pulmonary arteries (white arrowhead) are small in calibre; some small vasa vasora (black arrowhead) are also found as minor collaterals; c) follow-up CTPA obtained 16 months later clearly shows chronic thromboembolic pulmonary disease; d) slab volume-rendering image shows hypertrophy of right bronchial artery (arrows), which connects with vasa vasorum (black arrowhead) to supply distal pulmonary arteries (white arrowhead). AAO: ascending aorta; MPA: main pulmonary artery; DAO: descending aorta. Reproduced with permission from [124]. 
but not in the unobstructed territory (right upper lobe). To summarise, the connection of high-pressure systemic blood flow to the low-pressure pulmonary circulation is suspected to lead to muscular and fibrotic remodelling of pre-capillary arteries, capillaries and venules in obstructed vascular beds of human CTEPH [18], while pulmonary arterial overflow is held responsible for changes in small arteries and arterioles in unobstructed territories. In this piglet model of CTEPH, 6 weeks after surgical reperfusion of occluded left PA, secondary microvasculopathy regressed both in previously occluded and nonoccluded territories [17].

While Moser described plexiform lesions in lungs from CTEPH patients [121, 127], others have not observed these lesions that are typical for severe PAH [18]. Interestingly, recent reports suggest that plexiform lesions in $\mathrm{PAH}$ might represent bronchopulmonary shunts through abnormally developed anastomoses $[128,129]$.

Impact of proximal obstructive fibrotic clots and secondary microvasculopathy on haemodynamic severity

The presence of a severe microvasculopathy in a patient with CTEPH is usually suspected when the extent of mechanical obstruction by fibrotic organised clots does not correlate with the haemodynamic severity assessed by PVR. Some patients with CTEPH have very high PVR despite limited perfusion defects on lung scintigraphy, suggesting the presence of extensive microvasculopathy [130]. The haemodynamic impact of secondary microvasculopathy may also be evaluated with the pulmonary occlusion technique used to partition PVR into upstream resistance (due to proximal fibrotic organised clots) and downstream resistance (due to microvasculopathy). A lower pre-operative upstream resistance, suggesting extensive microvasculopathy, has been associated with a worse outcome after PEA and nonoperability [122, 131, 132]. However, the method is technically challenging and may lack discrimination power; it has not been incorporated into routine assessment.

\section{Clinical consequences of microvasculopathy}

The presence of a secondary microvasculopathy downstream of nonoccluded proximal pulmonary arteries may play a role in the progressive clinical deterioration of some patients in the absence of PE recurrence. The timing of the development of CTEPH microvasculopathy is unknown [121], but probably highly variable. The presence of a secondary microvasculopathy in CTEPH may explain persistence/recurrence of $\mathrm{PH}$ and poor outcome after PEA [133], and provides a rationale for the use of drugs approved for PAH.

Hypothetically, the occurrence of pulmonary oedema after PEA and BPA may be related to the restoration of normal flow to a previously occluded lung region with a microvasculopathy similar to pulmonary veno-occlusive disease and pulmonary capillary haemangiomatosis.

\subsection{Imaging of the pulmonary and bronchial circulations}

While CT can help in the visualisation of bronchial collaterals, MR can be used for both morphological evaluation as well as quantification of systemic to pulmonary shunt flow.

\section{CTPA}

As standard CTPA is optimised for pulmonary opacification, a longer delay from the injection of contrast medium to image acquisition is necessary for depicting bronchial collaterals (figure 10). ECG gating will permit sharper delineation of all vascular territories without pulsation artefacts. Limiting the acquisition to a single cardiac phase with prospective triggering can yield radiation doses that are comparable to non-ECG gated acquisition.

DECT improves vascular enhancement allowing assessment of pulmonary arterial perfusion including that of the collateral circulation; the latter is most conspicuous in the low kilovoltage component. PBV maps calculate the iodine distribution in the lung parenchyma and can be a surrogate marker of the underlying vascular reserve (figure 11).

\section{$M R /$}

MR-based phase-contrast measurements can be regarded as a link between macro- and microcirculation. High temporal resolution phase-contrast MRI allows for the calculation of bronchopulmonary shunting by measuring the flow difference between the pulmonary and systemic arterial circulation. The differential flow has been correlated with bronchial artery enlargement on CT, and the shunt size decreases in proportion to the technical success of PEA $[134,135]$.

Four-dimensional flow MRI is a time-resolved tridirectional velocity encoded cine sequence that can provide comprehensive assessment of blood flow with colour-coded multiplanar reformations, streamlines and velocity vectors. Using this technique, it has been established that in contrast to the normal central 

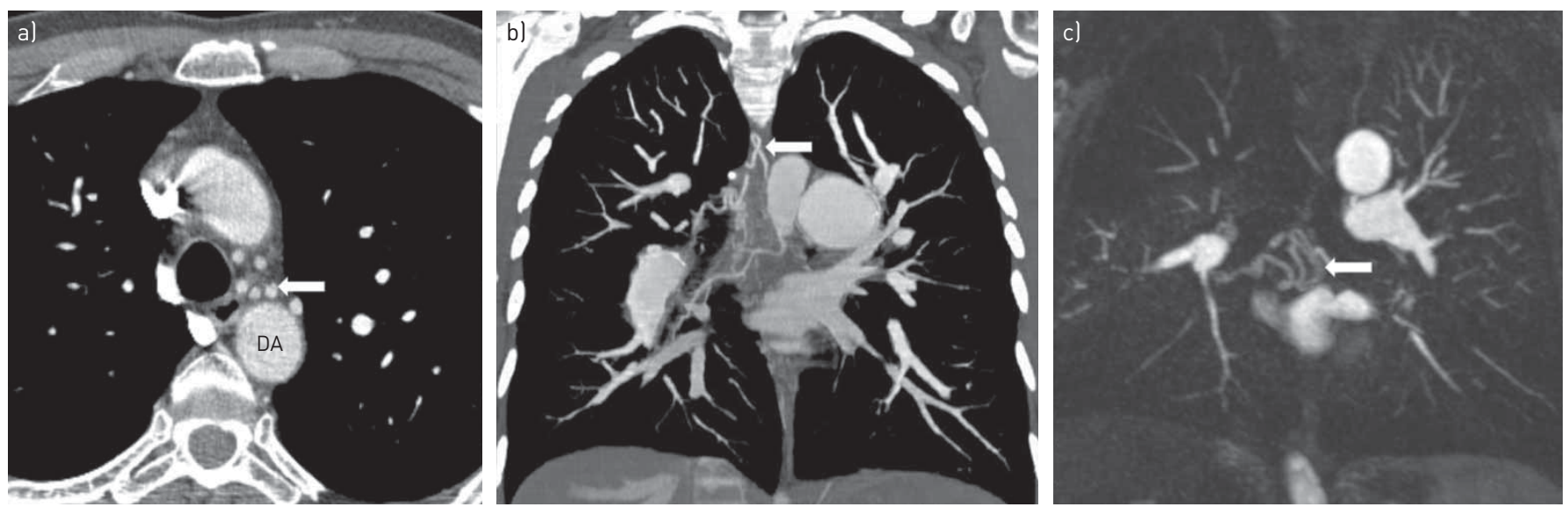

FIGURE 10 a) Axial and b) coronal computed tomography pulmonary angiography and c) magnetic resonance pulmonary angiography images show the enlarged bronchial arteries (arrows) in a chronic thromboembolic pulmonary hypertension patient. DA: descending thoracic aorta.

laminar flow along the main pulmonary artery, patients with $\mathrm{PH}$ have a vortical blood flow $>14.3 \%$ of the cardiac interval with a positive correlation between the duration of vortices and mPAP [136]. Diastolic vorticity was indicative of mild PH while systolic vorticity was seen in severe PH. Small CTEPH case series have shown improvement in flow vortices in the main pulmonary artery following successful BPA [137].

\subsection{The RV in CTEPH}

If exposed to similar loading conditions, the RV in patients with CTEPH is on average less adapted than in patients with PAH. This becomes clear by comparing haemodynamic data from CTEPH patients with PAH patients $[138,139]$. At a similar PVR, PAP is lower in CTEPH, indicative of a less adapted RV in comparison to $\mathrm{PAH}[139,140]$. In comparison to $\mathrm{PAH}$, mPAP is less in CTEPH patients. Several factors might contribute to this difference. First, patients with CTEPH are usually older than PAH patients. Second, the time course of increase in RV load may be different in CTEPH and PAH over time. CTEPH is thought to start with an acute episode of pressure overload at the time of acute PE, which may have an impact on RV remodelling. Finally, even when PVR is similar in a CTEPH and a PAH patient, the load for the RV may be different due to differences in wave reflection patterns [141].

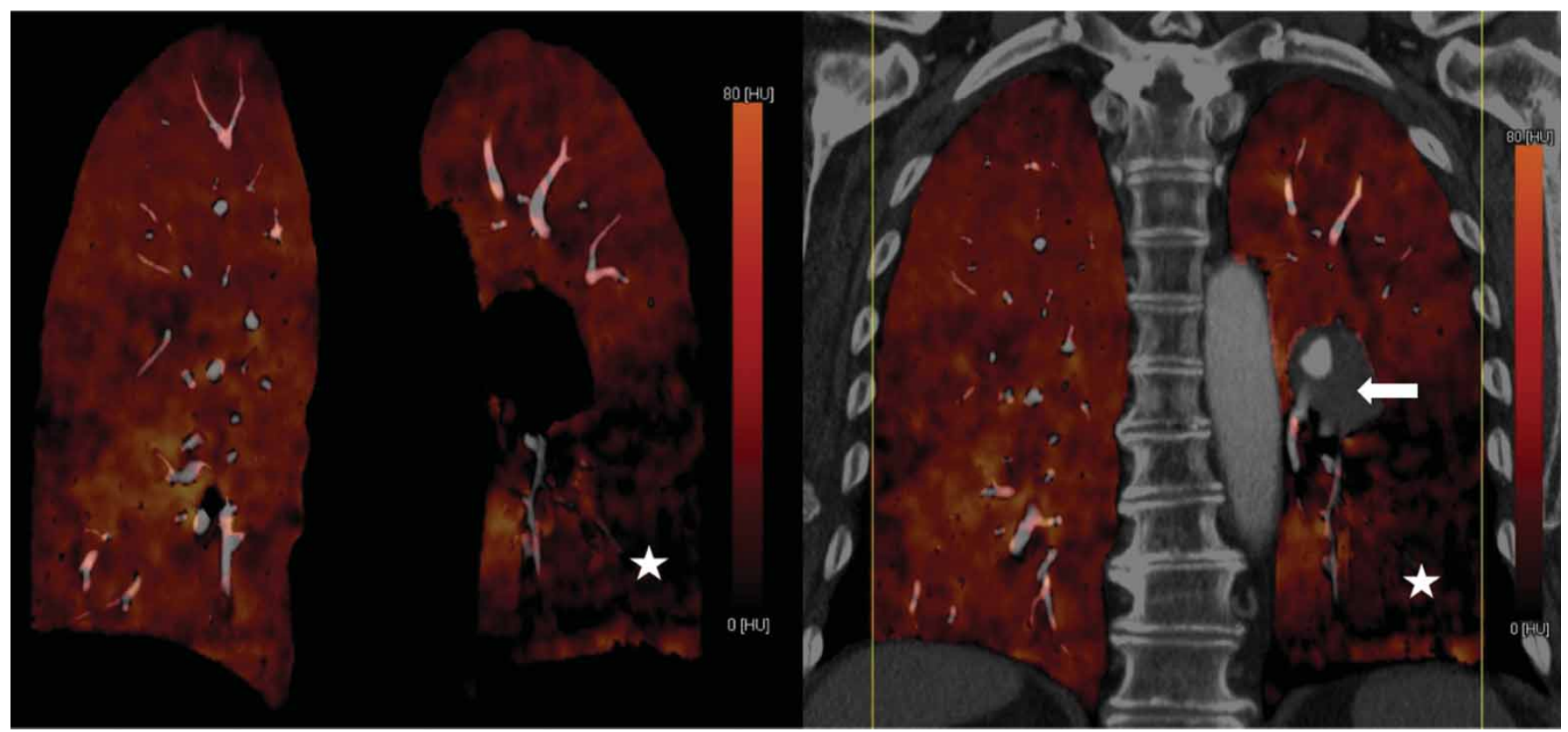

FIGURE 11 Coronal dual-energy computed tomography images with colour-coded perfused blood volume map in a chronic thromboembolic pulmonary hypertension case with left lower lobe occlusion (arrow) and corresponding large perfusion defect (star). 
Restoring the load of the RV in CTEPH by successful PEA brings a profound reduction of ventricular size and a recovery of systolic function, for the RV and LV [142]. What remains is an abnormal response to exercise $[7,143]$.

\section{Statements: pathophysiology}

5.1 Current knowledge suggests that two types of vascular lesions exist in CTEPH: proximal fibrotic obstruction in large elastic pulmonary arteries and a secondary microvasculopathy in pulmonary vessels $<500 \mu \mathrm{m}$.

5.2 Morphological delineation of pulmonary and bronchial circulation is possible with CTPA, while MRI permits both anatomical visualisation and semi-quantitative analysis of the extent of bronchopulmonary shunting. None of these techniques can quantify the microvasculopathy.

5.3 The right ventricle is less adapted in CTEPH than in idiopathic PAH, but recovers largely after successful PEA.

Proposal for future research in CTEPH pathophysiology

a) Search for biomarkers of fibrotic thrombus transformation

b) Explore modifiers of the transforming growth factor- $\beta$ pathway in thrombosis

\section{Pulmonary endarterectomy}

PEA (figure 12) is the treatment of choice for operable patients, inducing major haemodynamic and clinical improvements with a low early mortality $(<3 \%)$ in expert centres.

\subsection{What are the requirements for a PEA centre and surgeon?}

The 2015 ERS guidelines recommend that patients with CTEPH should be treated at an expert centre [1], with Jenkins et al. [144] attempting to define the characteristics of an expert centre in 2017. There should be experience of cardiothoracic surgery, including procedures requiring deep hypothermic circulatory arrest (DHCA), a $\mathrm{PH}$ service, expert diagnostic imaging and an expert multidisciplinary team including surgeons, radiologists, anaesthetists, intensivists, nurses, perfusionists, respiratory therapists and interventionalists able to provide BPA. This publication reiterated the expert consensus view that ideally there should be a limited number of PEA centres serving a population with a case volume of $>50$ procedures per annum, based on mortality data in small, intermediate and large centres participating in the International CTEPH registry [145], and $\geqslant 5$ years' experience, with the highest-quality centres achieving in-hospital mortality of $<5 \%$ and having the ability to perform distal endarterectomy and offer all three modalities of treatment. However, it is appreciated that this may not be possible for smaller countries. At the 2013 World Symposium on Pulmonary Hypertension (WSPH), the CTEPH task force recommended that PEA centres should have extracorporeal membrane oxygenation (ECMO) available to deal with the potential complications of surgery that can be unpredictable [19]. One of the largest experiences with ECMO has indicated that $3.7 \%$ of 829 patients required support with an in-hospital survival of $48.4 \%$ [146]. Surgeons should be experienced and trained either in cardiac or thoracic surgery. There are no data to suggest that one discipline is superior to the other, as long as thoracic surgeons are confident with the use of cardiopulmonary bypass (CPB). Most of the surgeons currently working in the largest PEA centres also have a background in intrathoracic transplantation. For a starting PEA programme, clinical practice suggests that mentored operating for 1 year and experience of $\geqslant 50$ procedures in a high-volume centre allows selected PEA surgery without any increase in morbidity or mortality. Adequate training of anaesthetists and intensivists is also mandated.

\subsection{How much anatomical overlap is there between PEA and BPA and which is the best treatment for segmental disease?}

The degree of anatomical overlap between what disease is accessible to PEA and BPA is thought to be considerable, although there are few published data and there are no RCTs comparing both techniques [147] (figure 13). There are no published data to indicate a threshold of accessibility from pre-operative imaging. Clinical experience indicates that both techniques can access subsegmental disease and both are viable treatment options for disease confined to segmental level. The surgical technique usually relies on traction of the wall lining more proximally and can therefore progressively advance the dissection plane into very small distal vessels until the specimen breaks away, beyond the point that can be visualised and instrumentation can directly reach. The type of lesion, as well as the location, is probably more important for BPA than PEA surgery. In an angiographic classification from Japan, it was found that outcomes were superior, and complications lower, for ring-like stenosis and web lesions compared with total occlusions [148]. Only PEA surgery can reliably tackle disease in the main pulmonary artery and its lobar branches as this more proximal disease usually includes organised thrombus and/or more bulky fibrotic webs. The intraoperative surgical 


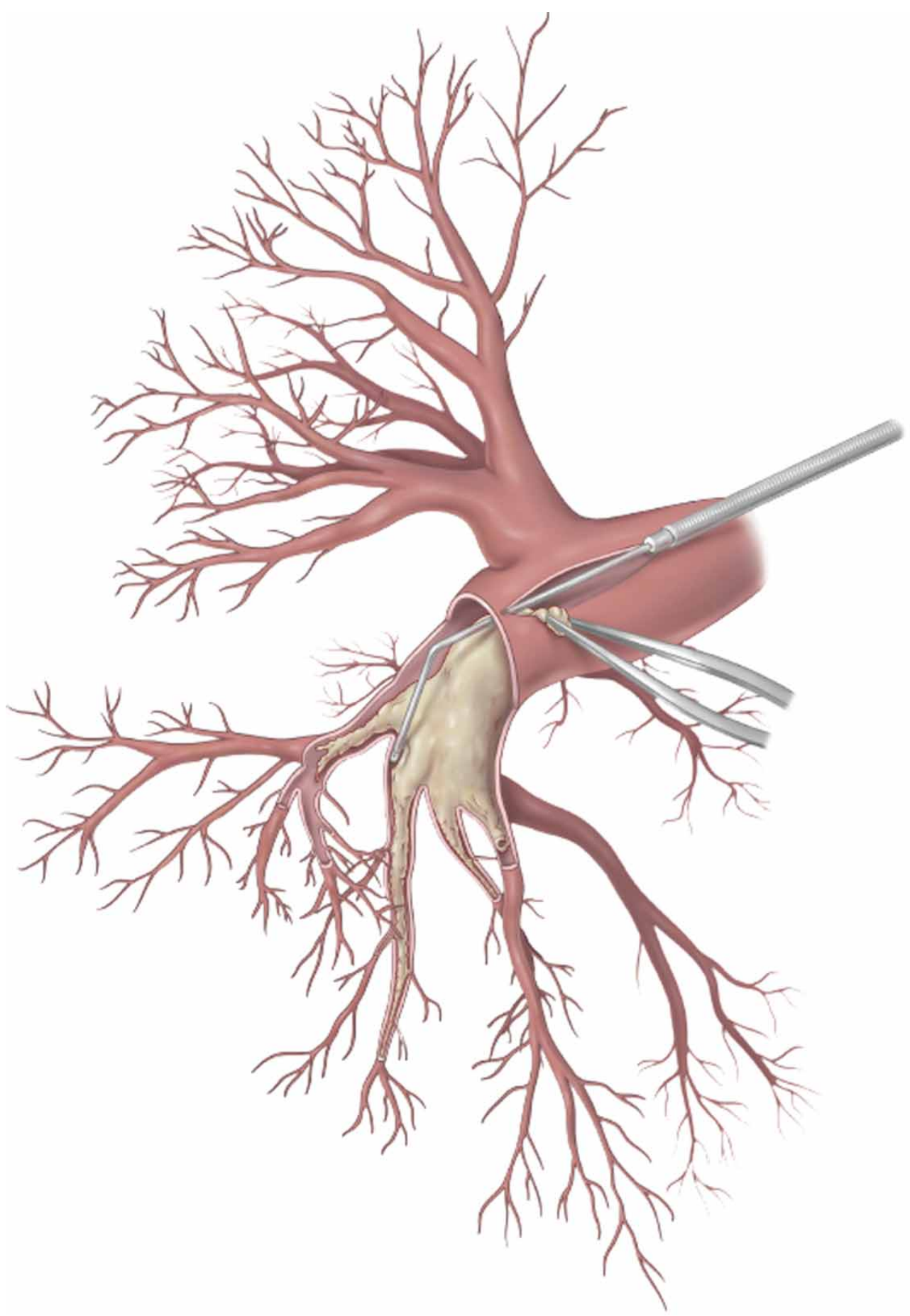

FIGURE 12 Pulmonary endarterectomy. The right pulmonary artery is opened, and the suction dissector is introduced between the artery wall and the fibrosis. Following the inside of the artery down to segmental and subsegmental level, the fibrotic material is subsequently freed from the wall and removed with forceps. Reproduced with permission from Maren Hötten, Master Thesis in Scientific Illustration "Chronic Thromboembolic Pulmonary Hypertension: Diagnosis and Treatment", University of Maastricht, 2016.

classification has been recently revised to recognise the level at which disease starts and separate segmental and subsegmental disease (levels III and IV) to reflect modern surgical practice [147]. One series has specifically investigated the outcome of PEA surgery for more distal disease [149]. In this series of 331 patients operated by a single surgeon between 2008 and 2013, PEA for Jamieson type 3 disease (segmental) was performed without an increase in operative mortality or morbidity. There were equivalent haemodynamic results at discharge, and importantly at 1 year, to those achieved for patients with more proximal disease. 


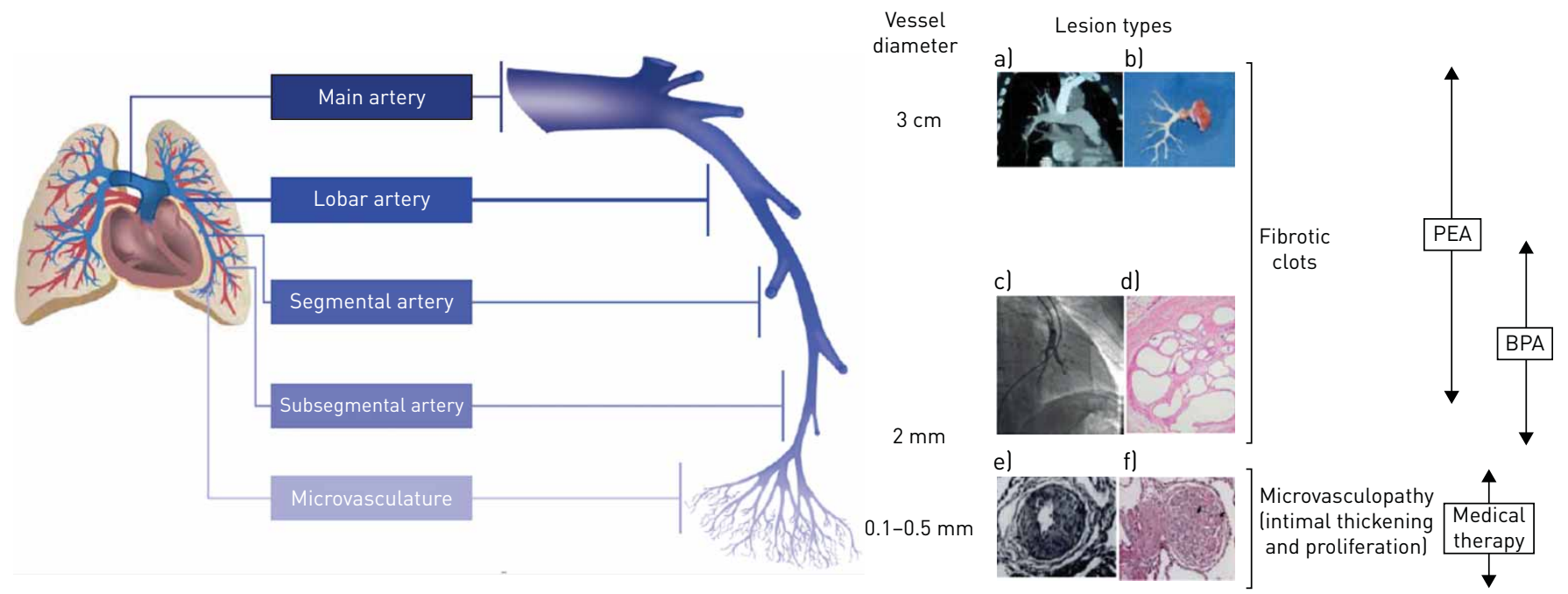

FIGURE 13 Respective targets for pulmonary endarterectomy (PEA) and balloon pulmonary angioplasty (BPA). a) Computed tomography scan of a pulmonary artery; b) organised fibrotic material removed during PEA; c) selective pulmonary angiogram of segmental and sub-segmental pulmonary arteries, showing irregular vessel contour and occlusion, typical of chronic thromboembolic pulmonary hypertension; d) microscopic examination showing a luminal filling defect with recanalised chronic thrombus (web lesion) and no evidence of vasculopathy in the subsegmental artery; e) intimal fibromuscular proliferation; f) plexiform lesion and vessel occlusion due to vasculopathy and proliferation. Reproduced with permission [147].

\subsection{What is a successful outcome after PEA surgery?}

There is no definition of success after PEA surgery, but patients would expect to survive the operation without cognitive dysfunction or major morbidity and recover with reduced PAP to gain improved functioning and quality of life that is sustained to prolong lifespan. It is the most effective treatment for CTEPH with the largest reduction in PAP and PVR, by $21 \mathrm{mmHg}$ and 561 dyn.s.cm ${ }^{-5}$, respectively, in a recent meta-analysis [150]. The benchmark of postoperative mortality $<5 \%$ was set by the International CTEPH registry that included 386 patients from 17 surgical centres [145]. A larger series from an experienced centre reported a mortality of $2.2 \%$ for a cohort of 500 patients [151]. More recently, the International CTEPH registry reported 3-year survival of $89 \%$ for the cohort of 404 operated patients compared with $70 \%$ for 275 patients who did not undergo surgery [99]. The longest complete follow-up is from the UK national series of 880 patients demonstrating $72 \%$ survival at 10 years for a mean age of 57 years. During extended follow-up, 49\% of deaths were unrelated to CTEPH [152]. Despite the need for DHCA to perform PEA surgery, cognitive function was shown to improve at 3 months and 1 year following surgery in a blinded RCT [153]. In addition, the same study reported improved quality of life using the specific PH Cambridge Pulmonary Hypertension Outcome Review (CAMPHOR) score. The longest follow-up of functional class post-surgery is in a series of 157 patients from the Pavia (Italy) centre, with improvement in functional class from $97 \%$ in class III/IV before surgery to $74 \%$ in class I at 4 years [154].

\subsection{How to manage residual/recurrent disease?}

There is no generally accepted definition of residual PH post-PEA. Different publications have used different criteria, with an overall estimate of $25 \%$ in a meta-analysis [150]. True recurrent CTEPH (i.e. evidence of improvement after PEA, followed by new symptoms and changes on imaging) is much rarer; only six out of 356 patients suffered from new PE after PEA in the UK series [152].

Management of significant residual PH can be challenging in the early post-operative period, and it is the most common cause of in-hospital mortality. ECMO can successfully help salvage selected patients with severe residual $\mathrm{PH}$ and haemodynamic collapse and allow bridging to lung transplantation, but experience is limited [146]. Medical therapy has not proven helpful in the early phase, with only one small RCT demonstrating reduced PVR with inhaled iloprost in the immediate post-operative period [155].

Patient long-term outcome after PEA is also affected by residual $\mathrm{PH}$, with data showing that mPAP $\geqslant 38 \mathrm{mmHg}$ and PVR $\geqslant 425$ dyn $\cdot \cdot \cdot \mathrm{cm}^{-5}$ at reassessment predict CTEPH-related deaths [152]. A suggested threshold to initiate drug therapy was mPAP $>30 \mathrm{mmHg}$ [152]. In operated patients with postoperative PVR $>300$ dyn $\cdot \mathrm{s} \cdot \mathrm{cm}^{-5}$, there is evidence for reduced PVR and improved 6-min walk distance (6MWD) with riociguat in the CHEST-1 study [156]. There is now some experience with the use of BPA to treat residual distal lesions post-PEA [157]. A further alternative in specialised centres is to consider redo-PEA and contemporary results in selected patients are satisfactory [158]. The 2018 WSPH treatment algorithm 
recommended the use of medical therapy and consideration of BPA or redo-PEA in patients with persistent symptomatic PH following PEA [20].

\section{Statements: PEA}

6.1 Requirements for a PEA centre are based on expert opinion only, but $\geqslant 50$ cases as centre annual volume, and a properly trained surgeon have been proposed.

6.2 There is probably considerable anatomical overlap between BPA and PEA, but segmental and subsegmental disease are suitable for surgery in expert, high-volume centres.

6.3 Successful outcome after PEA is multifactorial and assumes in-hospital mortality $<5 \%$, survival of $90 \%$ at 3 years, improved functional class and quality of life.

6.4 Residual PH after PEA presents in $\sim 50 \%$ and represents a target for recently introduced medical therapies and BPA. There is some evidence for their use if post-PEA mPAP remains $>30 \mathrm{mmHg}$ in the presence of symptoms.

Proposal for future research on PEA

a) Evaluation of the impact of pre-operative treatment (drug or interventional) on the outcome of PEA surgery

b) Comparison of the efficacy, risk and long-term outcome of PEA with BPA for segmental disease

\section{BPA}

7.1 What type of CTEPH patients are candidates for BPA? What is the role of pre-treatment with PH-targeted therapy prior to BPA?

For CTEPH patients deemed technically inoperable, percutaneous BPA (figure 14) has had a recent renaissance sparked by technical refinements introduced from Japan [159-161]. With now growing

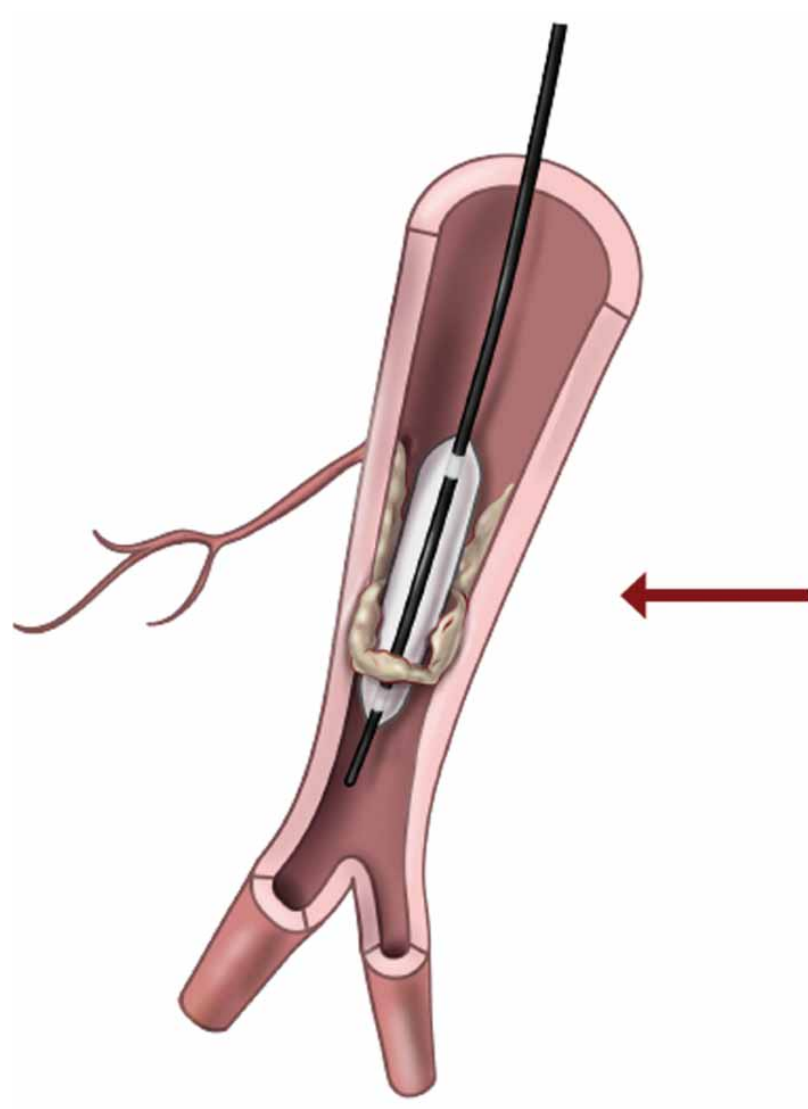

a)

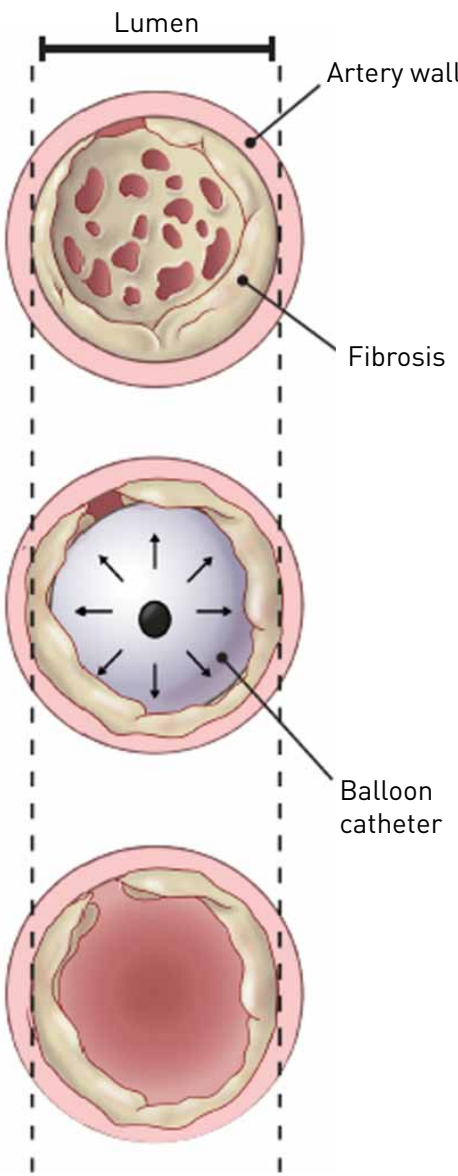

FIGURE 14 Balloon pulmonary angioplasty. a) A web-lesion in a subsegmental branch of the pulmonary artery; b) the wire is introduced between the fibrotic material and the balloon is inflated, leading to a rupture of the web; c) fibrotic material is connected to the vessel wall. Reproduced with permission from Maren Hötten, Master Thesis in Scientific Illustration "Chronic Thromboembolic Pulmonary Hypertension: Diagnosis and Treatment", University of Maastricht, 2016. 
worldwide experience with BPA, and together with advances both in PEA and medical therapy as overviewed in this series, there has been a major reset on how CTEPH cases are evaluated and treated.

BPA is not a replacement for PEA or targeted medical therapy in CTEPH. Instead, BPA fills an intervention gap for those CTEPH cases not eligible for PEA and may be considered in conjunction with either therapies as a complementary treatment modality [147, 162, 163]. Accordingly, the determination to proceed with BPA in any CTEPH case must not be made in isolation of these other established therapies [1]. The 2015 ESC/ERS guidelines had determined that BPA at the time garnered a IIb-C recommendation, meaning that it may be considered according to expert opinion and/or small studies [1]. This will require updating in the next guidelines as the intervention and our understanding of its role in CTEPH treatment have evolved at a rapid pace.

\subsection{What are the clinical outcomes and expectations of BPA for CTEPH?}

In experienced settings, BPA has produced improvements in pulmonary haemodynamics, 6MWD, functional class and quality of life $[162,164-166]$ by improving flow through the pulmonary circulation (figure 15). Additional improvements following BPA have been demonstrated in RV function by cardiac MR and in ventilatory efficiency by CPET $[57,168]$. The early reports of BPA benefit were initially from Japan, which raised the question of reproducibility and applicability to other populations or regions with active PEA programmes. The subsequent national BPA series published from Germany and France have reinforced support of BPA for CTEPH cases deemed inoperable $[169,170]$. The German series was the first major BPA report from a region with high-volume PEA experience [169]. They reported BPA improvements in $6 \mathrm{MWD}$ by $+33 \mathrm{~m}$ and reductions of $18 \%$ and $26 \%$ in mPAP and PVR, respectively. The subsequent French report represented the largest single-centre consecutive BPA series since programme initiation with 1006 interventions [170]. They reported 26\% and 43\% reduction in mPAP and PVR, respectively, with BPA. When they compared results between cases from the early programmatic period to the more recent cohort, the latter group had relatively larger magnitude of haemodynamic benefit. In addition, they observed lower complication rates in the more recent BPA cohort, supporting the recognition of a learning curve and the value of experience impacting on outcomes. Overall 6MWD improvement was $+45 \mathrm{~m}$ in this series. Accordingly, even from centres performing high volumes of PEA surgeries (which may influence the types of cases selected for BPA) the results of BPA have been encouraging.

\subsection{What are the important complications associated with BPA and their acceptable rates for an expert centre?}

Early pivotal experience with BPA reported in 2001 was notable for high complication rates, with $61.1 \%$ lung injury, $16.7 \%$ of patients in need of mechanical ventilatory support and one procedure-related mortality among the 18 patients [171]. In contrast, the new era of BPA has yielded safer results [164, 165]. The main contributors to this improved safety include 1) a better understanding of the mechanisms of BPA complications; 2) application of technical refinements introduced from expert centres; and 3) appreciation of the BPA learning curve and team experience (starting with patient selection) as critical factors in reducing and better managing the complications when they occur $[148,170,172]$. Contrary to
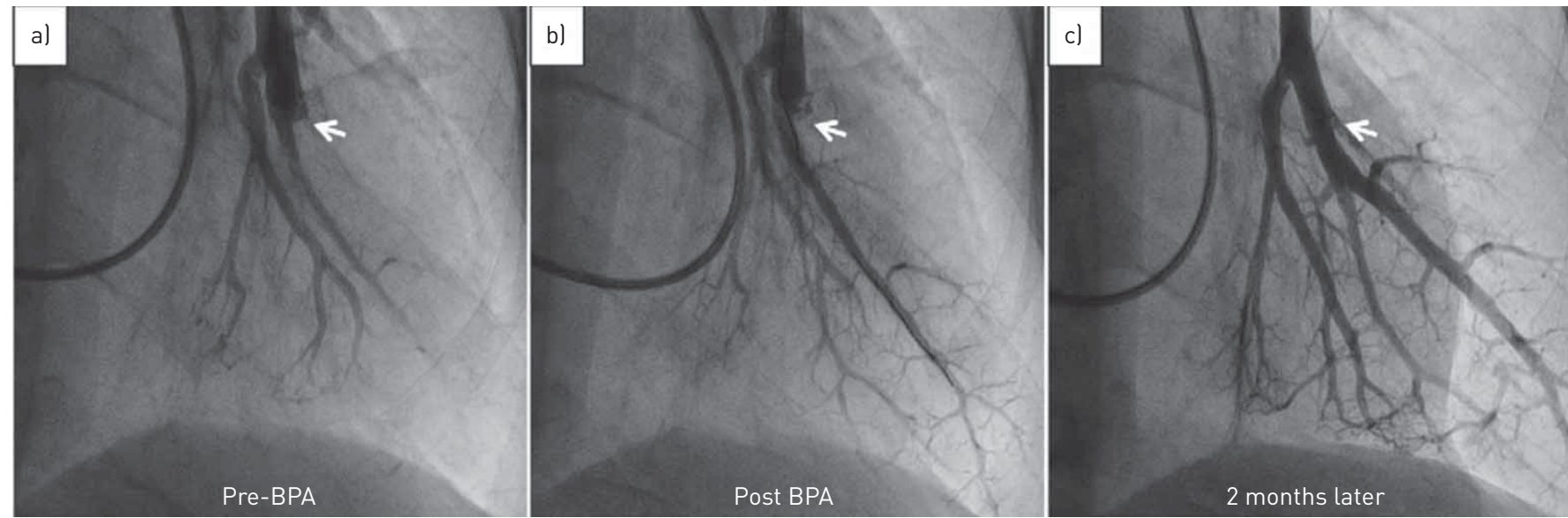

FIGURE 15 Pulmonary angiogram before and at two time periods after balloon pulmonary angioplasty (BPA) treatment. a) Pre-BPA; b) post-BPA; c) 2 months later. Reproduced from [167] with permission. 


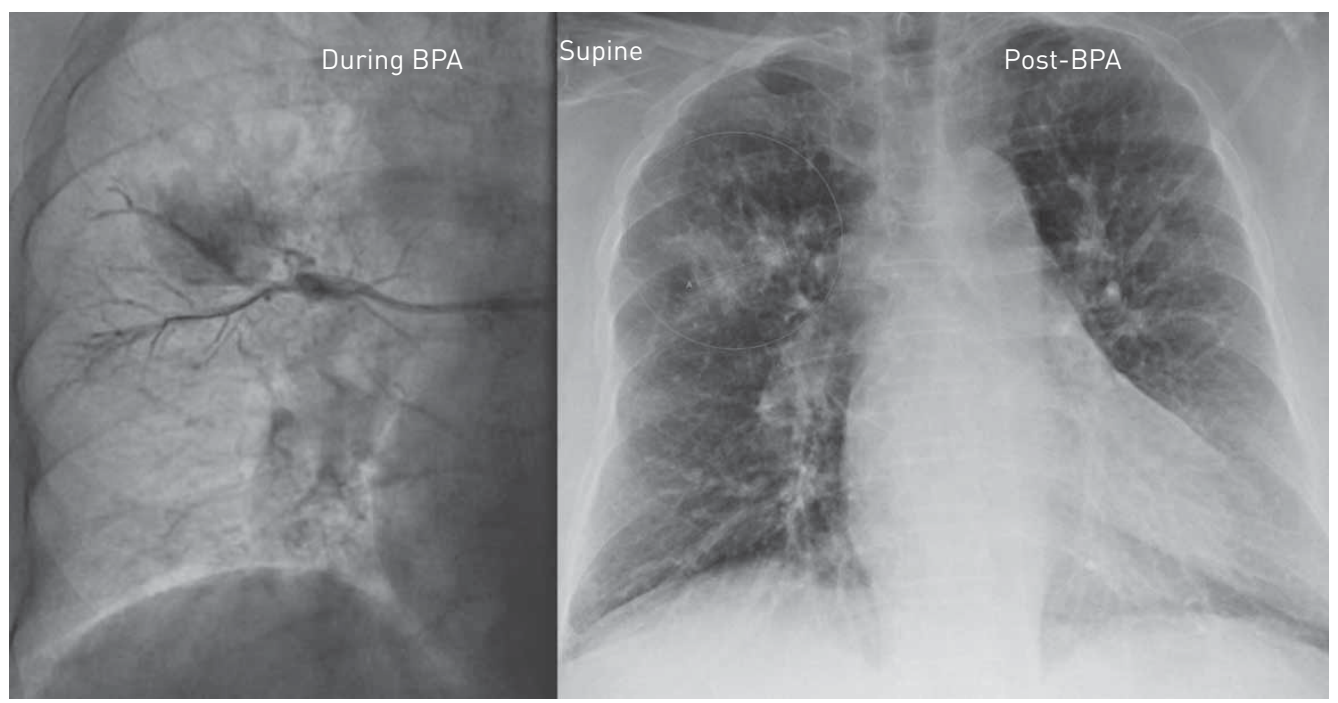

FIGURE 16 Vascular injury during balloon pulmonary angioplasty (BPA) signalled by new cough followed by haemoptysis resulting in temporary reversal of anticoagulation and end of BPA session.

earlier reports describing BPA-associated injury as reperfusion pulmonary oedema (as observed after PEA), there is now general consensus that the lung injury associated with BPA (figure 16) is instead from mechanical vascular injury during wire manipulation or balloon overdilation [172]. With such knowledge and with greater volume of procedures adding to experience level, BPA complications can be reduced to manageable rates $[170,172]$. In the German and French BPA series, the overall complication rates were $9.4 \%$ and $11.2 \%$ per procedure, respectively $[169,170]$. In the more recent cohort of 562 consecutive procedures from the French series (in the period described as being after the initial learning curve), the rate of complications had halved from an initial $15.8 \%$ to just $7.7 \%$ per procedure [170]. Results from different cohorts suggest that the rate of complication after BPA is closely related to the severity of $\mathrm{PH}$ at the time of BPA $[170,172,173]$.

BPA procedures still carry a risk of mortality. The two largest published series, the French report and the multicentre Japanese registry, the BPA mortality rates were $3.8 \%$ and $3.9 \%$, respectively $[73,170]$. Currently, there is an ongoing International BPA Registry which aims to better define and compare results and complications in a prospective fashion from established BPA centres across Japan, Europe and USA (NCT03245268). This registry has potential to shed some valuable light on the heterogeneity in current BPA practices across regions and between centres.

\subsection{What are the requirements to establish a BPA centre? What criteria and parameters define an expert BPA centre?}

There are numerous key limitations regarding BPA and its treatment of CTEPH. The reported safety and efficacy of BPA come from expert centres performing these and other CTEPH treatment modalities on a routine basis. Since success of BPA starts with proper patient selection and centre BPA experience, the observed results may not be applicable to all centres. The procedure remains risky even in experienced hands and should be confined to expert centres [147]. Furthermore, the benefits of BPA need to be interpreted with some caution, since there is still paucity of long-term results beyond 5 years [174, 175]. Although there are some encouraging early observations on improved survival with BPA in the inoperable CTEPH population [176], whether BPA-treated patients sustain an excellent long-term survival similar to that observed following successful PEA remains unknown [99, 152]. Furthermore, unlike PEA, BPA requires multiple sessions. There is no consensus or guidelines on treatment end-point or continuation of BPA efforts. Lastly, the addition of BPA provides a more complex treatment decision for any individual CTEPH patient. The ideal sequence and timing of combination or stepwise approach incorporating all available treatment modalities is yet to be determined and may need to take into consideration centre- or region-specific factors.

Although the 2015 ESC/ERS guidelines proposed preliminary definitions of an expert PH referral centre [1], and the topic was deliberated by the 2018 WSPH task force on CTEPH in the context of PEA and BPA centres [20], we currently do not have adequate evidence to define expertise based solely on procedural volume. Nevertheless, and similar to PEA surgery $[145,151]$, there is consensus and demonstrable correlation between BPA outcomes with level of BPA experience [170]. Of note, percutaneous non-BPA interventional experience, while helpful, does not ensure success with BPA given 
the differences between the systemic and pulmonary vasculature, nature of complications and targeted pathology [170]. Furthermore, and worth re-emphasising, aside from technical proficiency that comes with procedural volume, success of BPA and individual case outcome also relies importantly on proper patient selection within the context of a multidisciplinary CTEPH team considering all current and available treatment modalities [1, 170]. For a starting BPA programme, clinical practice suggests that direct mentorship from a high-volume centre followed by experience of $\geqslant 50$ procedures allows results without excessive morbidity or mortality.

Within a relatively short period of time, BPA has evolved from an experimental modality to becoming an established treatment option for inoperable CTEPH. Looking ahead, thresholds for successful BPA outcomes, acceptable rates of complications and optimal incorporation with the other treatment modalities need to be investigated and established. This will require concerted effort and collaboration among CTEPH centres.

\section{Statements: BPA}

7.1 Inoperable CTEPH patients can benefit from BPA. Optimal CTEPH treatment requires a multidisciplinary team approach considering PEA, medical therapy and BPA.

7.2 Long-term results after BPA are available out to 8 years after the procedure. Safety and efficacy of BPA correlate with centre experience.

7.3 Vascular injury rather than reperfusion pulmonary oedema is the likely cause of any severity of lung injury after BPA.

7.4 Similar to PEA, proper training in a high-volume centre is critical for BPA.

Recommendations for research and training

a) Define the role of PH-targeted medical therapy in relation to BPA

b) Identify treatment goals and key end-points for completing BPA interventions per individual patient

c) Create standardised definitions of BPA complications

d) Establish criteria or expected outcome thresholds to be recognised as an expert BPA centre

\section{Medical treatment}

\subsection{Long-term anticoagulation: what is the current standard of care in CTEPH IVKAs versus NOACs)?}

Besides lifelong anticoagulation, CTEPH basic therapy includes diuretics in case of right heart failure and oxygen in hypoxaemic patients [92, 93]. Traditionally, VKAs have been the mainstay of anticoagulant treatment in PAH and CTEPH, but NOACs are increasingly used [90, 92, 93, 177]. There are no strong data on the efficacy and safety of NOACs in CTEPH. In addition, robust information is needed on drugdrug interaction between NOACs and pulmonary vasodilators used in CTEPH. Because NOACs are regularly used in some centres, registry-based information has been released recently [90, 177].

Consecutive CTEPH patients undergoing PEA between 2007 and 2018 at the Royal Papworth Hospital (Cambridge, UK) have been included in a retrospective analysis (794 patients were treated with VKAs and 206 with NOACs following PEA) [90]. Haemodynamic and functional improvements were observed in both groups following PEA. In terms of safety, major bleeding events were equivalent in those treated with VKAs and NOACs $(0.7 \%$ per person-year), but the VTE recurrence rate was proportionately higher with NOACs (4.62\% per person-year) than VKAs (0.76\% per person-year), although survival did not differ [90]. Additionally, in a retrospective analysis of 405 consecutive PEA cases, CTEPH patients on NOACs were twice as likely to have associated acute or subacute thrombi removed at the time of surgery compared with those on conventional anticoagulant therapies [178].

In the setting of BPA, continued VKA treatments without interruptions appear to be practiced by the majority of expert centres.

EXPERT, an international, multicentre, prospective, noninterventional registry study, assessing riociguat safety in clinical practice (NCT02092818) has recently released data from PAH/CTEPH patients who received riociguat with either concomitant NOACs $(n=227)$ or VKAs $(n=617)$ at baseline [177]. In terms of adverse events, haemorrhages were reported in 26 (11\%) patients in the NOACs group and $81(13 \%)$ patients in the VKAs group, and thromboembolic events were reported in nine (4\%) and 24 (4\%) patients, respectively. In terms of serious adverse events, haemorrhages were reported in $13(6 \%)$ patients in the NOACs group and $39(6 \%)$ patients in the VKAs group, fatal haemorrhages in one $(0.4 \%)$ and six $(1 \%)$ patients, respectively, and thromboembolic events were reported in eight (4\%) and 23 (4\%) patients, respectively. Thus, serious bleeding/thrombotic events were uncommon, and their frequency was similar between PAH/CTEPH patients receiving concomitant NOACs or VKAs at baseline. Rates of serious 
haemorrhage or thromboembolic serious adverse events were similar with baseline NOACs or VKAs for PAH and CTEPH, separately [177].

A subgroup of CTEPH patients present with antiphospholipid syndrome. In that setting, VKAs (international normalised ratio target 2.5) have been demonstrated as carrying more benefit and less risk than NOACs in high-risk patients with thrombotic antiphospholipid syndrome (triple-positive for lupus anticoagulant, anticardiolipin, and anti- $\beta_{2}$-glycoprotein I antibodies of the same isotype) [94].

\subsection{PH medication: what is the available evidence on approved and not (yet) approved PH medications in CTEPH?}

Acute pulmonary vasodilator testing is not recommended in $\mathrm{CTEPH}$, even if $\mathrm{PH}$ reversibility could be a predictor of residual PH after PEA [179], and there is no indication of calcium channel blocker use in this condition [180]. In CTEPH, there is evidence of small pulmonary vessels disease indistinguishable from that of PAH, and similar treatment targeting the endothelin-1, nitric oxide and prostacyclin pathways have been studied [120].

In the CHEST-1 RCT of 261 patients with inoperable CTEPH or persistent/recurrent PH after PEA, an oral guanylase cyclase stimulator (sGC; riociguat, targeting the nitric oxide pathway) significantly increased 6MWD and reduced PVR, as compared to placebo [156]. These data led to the approval of riociguat for patients with inoperable CTEPH or persistent/recurrent PH after PEA. Currently, riociguat is being tested in RCTs, addressing its efficacy and safety as bridging therapy for patients scheduled to undergo PEA (NCT03273257). Another study compares riociguat to BPA in inoperable patients (NCT02634203).

Sildenafil, a phosphodiesterase type 5 inhibitor (PDE5i), has been tested in a small RCT in inoperable CTEPH [181]. Sildenafil (40 mg three times daily) or matching placebo was given to nine and 10 inoperable CTEPH patients, respectively. At 12 weeks, no improvement was seen in the primary end-point (6MWD) despite a positive effect on PVR [181]. This pilot study was insufficiently powered to test the primary end-point, and larger-scale trials of sildenafil in inoperable CTEPH have not been performed since then. Sildenafil is thus not approved for CTEPH.

The dual endothelin receptor antagonist (ERA) bosentan showed no improvement in 6MWD (despite a positive effect on PVR) in an RCT of 157 patients with inoperable CTEPH or persistent/recurrent PH after PEA (BENEFIT study) [182]. Bosentan is thus not approved for CTEPH. Safety and efficacy of the dual ERA macitentan was assessed in a phase 2 RCT, showing significantly improved 6MWD and PVR compared to placebo in $80 \mathrm{CTEPH}$ patients adjudicated as inoperable (MERIT-1) [183]. Interestingly, this was the first RCT in CTEPH assessing the combination of an ERA with other medications approved for $\mathrm{PAH}$, but excluding riociguat (61\% of patients received off-label PDE5i or oral/inhaled prostanoids).

Besides off-label use, there is a single RCT studying prostacyclin analogues in inoperable CTEPH (CTREPH study) [184]. This RCT showed that long-term subcutaneous treprostinil is safe and effective in 105 severe inoperable CTEPH patients, with improvements of 6MWD, PVR, functional class and NT-proBNP. In this study, $30 \%$ of patients received other pulmonary vasodilator therapies. These data led to the marketing authorisation of treprostinil for patients with inoperable CTEPH or persistent/recurrent PH after PEA.

\subsection{Combination therapy: is there a role for initial or sequential combination therapy in CTEPH?} Sequential combination therapy of PH medications was allowed in CTEPH RCTs such as MERIT-1 [183] and CTREPH [184]. By analogy with PAH [177], initial dual oral combination therapy is currently considered in expert centres for patients with severe CTEPH. This is particularly true in inoperable CTEPH prior to BPA, in order to perform BPA in patients with less severe haemodynamic impairment and presumably at lower risk of procedure-related complications. Recent single-centre registry data of initial dual oral combination therapy using an ERA in association with either a PDE5i or riociguat in inoperable CTEPH patients have been reported [185]. These data indicate that initial oral dual combination therapy resulted in more pronounced reduction in PVR, when compared to monotherapy.

\subsection{De-escalation: when and how to stop/reduce PH drugs after successful BPA/PEA?}

Most CTEPH patients improve markedly clinically and haemodynamically after BPA and/or PEA [73, 170]. Lifelong anticoagulation is mandatory even if PEA and/or BPA have normalised pulmonary haemodynamics at rest, while the use of diuretics and oxygen are often no longer needed. Similarly, stopping or de-escalating PH medications may be considered after successful BPA and/or PEA. The Japanese multicentre registry for BPA indicates that $\mathrm{PH}$ medications were used in $72 \%$ of 308 cases before BPA, and $45 \%$ of 196 cases at last follow-up (at a mean \pm SD $425 \pm 281$ days after the final BPA procedures) [73]. In the French experience, $62 \%$ of 181 patients undergoing BPA received $\mathrm{PH}$ medications prior to 
BPA (26.6\% monotherapy, 28.3\% double combination and $7.1 \%$ triple combination therapy) [170]. The ongoing International BPA Registry will provide long-term information on this procedure and more details on the use of PH medications before and after BPA (NCT03245268).

\section{Statements: medical treatment}

8.1 According to current guidelines, CTEPH patients should be treated with lifelong anticoagulation; VKAs are the mainstay of anticoagulant treatment in CTEPH, but NOACs are increasingly used, with no safety issues reported yet. Antiphospholipid syndrome is a contraindication to NOACs.

8.2 Riociguat, an oral guanylate cyclase stimulator, and treprostinil, a subcutaneous prostacyclin analogue, are approved for patients with inoperable CTEPH or persistent/recurrent $\mathrm{PH}$ after PEA; other $\mathrm{PH}$ medications have been tested in CTEPH and are used off-label.

8.3 Oral combination therapy is a common practice in CTEPH patients with severe haemodynamic compromise.

8.4 Withdrawal of PH medications is usually considered after successful BPA and/or PEA.

\section{Proposal for future research on medical therapy for CTEPH}

a) Prospective registry data and/or studies to evaluate the safety of NOACs in CTEPH are encouraged

b) Studies testing the efficacy and safety of initial dual oral combination therapy are needed in CTEPH patients with severe haemodynamic compromise

c) A dedicated trial of carefully monitored withdrawal of $\mathrm{PH}$ medications should be considered after successful BPA and/or PEA

\section{Multimodality treatment}

9.1 What are the criteria for selecting candidates?

Many patients may have mixed anatomical lesions with lobar, segmental and microvascular lesions. With three effective treatments now available, the current treatment paradigm has evolved to include a multimodal approach to management. Such an approach involves employing combinations of PEA, BPA and pharmacological therapy to target proximal lesions, distal lesions and microvasculopathy, respectively. Currently, there is no guidance or standardisation regarding assessment of suitability for multimodal therapy and patient selection is performed on a case-by-case basis in expert centres through a multidisciplinary team approach.

Given the various treatment options for CTEPH, lung transplantation is now rarely considered in this group of patients.

\subsection{Is there a potential role for PH-targeted medical therapy prior to PEA in patients with operable CTEPH?}

The pre-operative PVR has been shown to be a predictor of post-operative mortality $[145,151,186]$. In the International CTEPH Registry, including 386 operated patients, an almost linear relationship between pre-operative PVR and in-hospital mortality was observed [145]. The use of PH-targeted therapy in patients with high pre-operative PVR may improve pulmonary haemodynamics before surgery and potentially reduce the morbidity and mortality associated with PEA. Two case series examined a total of 21 patients treated with intravenous epoprostenol prior to PEA. An improvement in pre-PEA haemodynamics, but no change in post-PEA haemodynamics was observed compared to controls [187, 188]. In a randomised, controlled single-blind study including 25 patients with total pulmonary resistance (TPR) $>900$ dyn $\cdot s \cdot \mathrm{cm}^{-5}$, pre-treatment with bosentan for 16 weeks demonstrated a reduction in pre-PEA TPR and mPAP, but no difference in post-PEA haemodynamics compared to controls [189]. In this study, four patients died during or after PEA $(n=1(7.6 \%)$ in the bosentan group and $n=3(25 \%)$ in the control group). In a retrospective analysis of CTEPH patients referred to the University of California at San Diego, JENSEN et al. [103] assessed the effects of pre-PEA PH-targeted therapy on pre-PEA haemodynamics and post-PEA outcomes. For this analysis, the subjects were divided into two groups: those who were on $\mathrm{PH}$-targeted therapy before referral for PEA and those who were not (control group). The authors showed that the use of $\mathrm{PH}$ medications delayed the time to referral for PEA with only a minimal improvement in pre-operative mPAP. In addition, their use did not result in any differences in post-PEA haemodynamics or outcomes [103]. In the International CTEPH Registry mentioned earlier, 29\% of operated CTEPH patients had received $\mathrm{PH}$-targeted therapy prior to PEA. These patients had more severe haemodynamic profile than those who did not receive bridging therapy, while no significant difference was observed in either post-operative PVR or post-operative complications between the two groups. More interestingly, the authors found that bridging therapy with $\mathrm{PH}$-targeted drugs was associated with an increased risk of death [99]. However, a recently published retrospective, single-centre analysis suggested that the use of 
targeted therapy was associated with a significantly better 1 -year survival (89.1\% versus $64.1 \%)$ in patients with a more severe haemodynamic impairment [190], relaunching the discussion on whether PH-targeted therapies before surgery in patients with operable CTEPH may result in better survival. Unfortunately, the randomised, placebo-controlled, multicentre PEA bridging study looking at safety and efficacy of riociguat prior to PEA in patients with operable CTEPH and high pre-operative PVR $\left(>800 \mathrm{dyn} \cdot \mathrm{s} \cdot \mathrm{cm}^{-5}\right)$ had to be terminated because of poor recruitment (NCT03273257).

\subsection{Is there a role for combined PEA and BPA in patients with CTEPH?}

Some CTEPH patients may have mixed anatomical lesions with surgically accessible lesions for one lung and inoperable lesions affecting the contralateral lung. Depending on the severity of $\mathrm{PH}$, low-risk patients with mixed anatomical lesions may undergo PEA, and if necessary, BPA during follow-up after surgery. High-risk patients (with high pre-operative PVR) might benefit from a combined therapy with BPA (prior to or at the same time as surgery) and PEA to decrease the surgical risk and improve the final result. However, data regarding the impact of this strategy on post-operative outcome are very limited so far and further studies are needed to confirm its clinical relevance $[163,191]$.

In large PEA cohorts, it has been shown that a significant proportion of patients may have residual or recurrent PH following PEA, which can adversely affect their quality of life and outcome $[145,151,152,186]$. Three case series, including a total of 34 patients, have specifically investigated the safety and efficacy of additional BPA for the treatment of residual or recurrent PH after PEA [157, 192, 193]. The majority of patients were treated with PH-targeted drugs before BPA and the time between PEA and first BPA session ranged from a few months to several years. In these case series, BPA improved haemodynamics and exercise capacity with a rate of complication that was similar to that observed in inoperable CTEPH patients treated with BPA.

BPA has been reported as a rescue treatment for early failure of PEA, without producing convincing results [194, 195].

\subsection{Is there a role for combined use of PH-targeted therapy and BPA in patients with inoperable CTEPH?}

Most BPA series reported to date included a high proportion of patients who were treated with $\mathrm{PH}$-targeted therapy prior to BPA. In initial Japanese reports, $\mathrm{PH}$-targeted drugs were initiated to improve the safety of BPA by optimising pre-BPA haemodynamics $[159,161]$. This strategy was based on the finding of FEINSTEIN et al. [171] who first showed that a pre-BPA mPAP $>35 \mathrm{mmHg}$ was correlated with the occurrence of lung injury known as one of the main complications related to BPA.

This approach has been applied in more recent studies, although there have been no clinical trials specifically designed to investigate its effectiveness [49, 73, 169, 170, 172, 175]. In two of these studies the relationship between high pre-BPA mPAP and occurrence of lung injury was also demonstrated, thus supporting the use of pharmacological treatments prior to BPA [170, 172]. The effects of this strategy in inoperable CTEPH have been examined in two studies. In a single-centre study, consecutive patients with inoperable CTEPH were treated with medical therapy prior to BPA, which resulted in improved cardiac index, PVR and exercise capacity, but little change in mPAP [175]. The patients then underwent BPA and had significant further reductions in MPAP and PVR and further improvement in symptoms and exercise capacity. In an observational cohort study, W IEDENROTH et al. [196] investigated more specifically the effects of a sequential treatment with riociguat and BPA. The authors showed that riociguat improved haemodynamics in patients eligible for BPA and that BPA led to further improvement in exercise capacity and haemodynamics. These data suggest that medical therapy combined with BPA may have an advantage over medical therapy alone, although larger prospective studies are needed to confirm this finding.

\section{Statements: multimodal treatment}

9.1 There is no consensus on eligibility criteria for multimodal therapy, which is dependent on the expertise and judgement of each individual CTEPH centre. Patient selection for multimodal therapy is performed in expert centres through a multidisciplinary team approach.

9.2 There is no convincing evidence that pre-operative pharmacological treatment is beneficial in patients with operable CTEPH. Nevertheless, $\mathrm{PH}$-targeted drugs as bridging therapy to PEA is sometimes considered in selected high-risk patients (with high pre-operative pulmonary vascular resistance) after multidisciplinary team assessment in expert centres.

9.3 The role of BPA as bridging therapy prior to PEA in patients with mixed surgically accessible and inaccessible lesions needs to be explored. The potential benefit of hybrid procedures simultaneously combining PEA and BPA in such patients needs to be confirmed by further studies. There is insufficient evidence to support the use of BPA as a rescue procedure for early failure of PEA. 
Additional BPA, most often in combination with $\mathrm{PH}$-targeted medical therapy, is common practice for patients with persistent symptomatic $\mathrm{PH}$ after PEA.

9.4 Most task force members use $\mathrm{PH}$-targeted therapy prior to BPA to improve the safety of BPA, although there have been no clinical trials investigating the benefit of this approach on the rate of BPA-related complications. Some data suggest that the combination of PH-targeted therapy and BPA provides better results than medical therapy alone.

Proposal for future research in multimodal treatment

Studies are needed to investigate the most appropriate approach to combination of BPA and PEA

\section{Rehabilitation}

\subsection{What is the cause of exercise limitation in CTEPH?}

The role of rehabilitation in PAH and CTEPH has been well established [197, 198]. Despite the fact that the beneficial effects of rehabilitation are not fully understood, increased capillarisation of the skeletal muscles as well as improvement in RV function as a consequence of rehabilitation have been measured in both animal models of pulmonary hypertension and PAH patients [198, 199]. Although exercise physiology is slightly different in CTEPH in comparison to PAH [200], it is reasonable to believe that the positive effects of rehabilitation are similar for both conditions. Indeed, in the first randomised large trials showing the beneficial effects of rehabilitation, PAH and inoperable CTEPH patients were grouped together [197, 198].

\subsection{Is rehabilitation safe in CTEPH?}

In a further study, NAGEL et al. [201] showed that rehabilitation was effective and safe in a group consisting of inoperable CTEPH patients.

\subsection{What are the requirements for rehabilitation in CTEPH?}

Most of the pivotal trials on rehabilitation in CTEPH and PAH were based on in-hospital training in tertiary referral set-ups. Small nonrandomised studies showed that home-based rehabilitation might also be an effective and safe option [202]. However, exercise training in $\mathrm{PH}$ should be individually adjusted and closely supervised by both $\mathrm{PH}$ experts and physiatrists who are experienced in the rehabilitation of severely compromised patients. Patients should be on optimised targeted treatment and in a stable clinical condition before performing a supervised rehabilitation programme [1].

\subsection{Is rehabilitation clinically relevant and safe pre- and post-PEA and BPA?}

Another potential role of rehabilitation is in the recovery phase after PEA and BPA. Until now, the potential role of rehabilitation after PEA has only been studied in a small nonrandomised retrospective study [203]. Although significant changes in 6MWD were observed during the 3-month rehabilitation period, it remains unclear whether these beneficial effects were the sole consequence of rehabilitation. In a prospective randomised study FUKUi et al. [204] studied the impact of rehabilitation directly after BPA. Although the patient number was small, results showed that the combined approach of BPA and rehabilitation programme was more successful than BPA alone. Based on these studies, a carefully monitored, low-dose rehabilitation programme after surgical or BPA treatment of CTEPH patients might be considered standard of care.

10.5 Is there a potential role for rehabilitation in CTEPD patients without PH?

Although of interest, the role of rehabilitation in symptomatic CTEPD patients has not been studied so far.

\section{Statements: rehabilitation}

10.1 Although exercise physiology is slightly different in CTEPH in comparison to PAH, it is reasonable to believe that the positive effects of rehabilitation are similar for both conditions.

10.2 Rehabilitation seems to be effective and safe in inoperable CTEPH patients.

10.3 Although most of the pivotal trials on rehabilitation in CTEPH and PAH were based on in-hospital training in tertiary referral set-ups, small nonrandomised studies have shown that home-based rehabilitation might also be an effective and safe option.

10.4 A carefully monitored, low-dose rehabilitation programme of CTEPH patients after PEA or BPA might be considered standard of care.

Proposal for future research in CTEPH rehabilitation

a) Larger-scale RCTs are needed to define the optimal training methodology and point in time (e.g. time after PEA) of exercise training in CTEPH

b) Studies on mechanisms of action of exercise training after PEA are needed 


\section{Global research lines}

\subsection{Biobank and basic science}

Studies investigating the underlying molecular and genetic alterations responsible for contributing to the development of CTEPH are sparse, have included small numbers of patients, and mostly lack the inclusion of independent validation cohorts [205]. In rare conditions such as CTEPH, new genetic insights and basic science progress can be achieved only by international collaboration and establishing biobanks/ biorepositories allowing researchers access to data representing a large number of patients. Currently there is no established international CTEPH biobank; however, CTEPH patients have been included into $\mathrm{PH}$ projects such as PVDOMICS [206]. International collaborations/biobanks between PAH centres which have been successful in establishing genetic inside to PAH have been extended looking into CTEPH through genome-wide association studies and circulating -omics studies [207].

Treatment with PEA/BPA offers a unique opportunity to study the reversibility of disease-associated phenotypes. Changes in metabolic and proteomic profiles in response to PEA/BPA, improvement in pulmonary haemodynamics, linking with clinical outcomes and therefore tracking -omic changes in response to therapeutic interventions could be a valuable outcome measure. Integration of clinical and -omic measurements and examining for clusters that identify subgroups of CTEPH may identify clinically valuable diagnostic signatures [208].

\subsection{Paediatric CTEPH}

While CTEPH is a rare disease in adults, it is quite exceptional in the paediatric population. There are very few data published on chronic thromboembolism and only few case reports/series with CTEPH [209211]. This important knowledge gap should be addressed by international prospective registries.

\section{Statements: global research lines}

11.1 To achieve new genetic insights and basic science progress, international collaboration through biobanking should be established to allow researchers access to the data which represents a large number of patients. Integration of clinical and -omic measurements will identify clinically valuable diagnostic signatures and pathways for potential future interventions.

11.2 There is a need to address CTEPH in paediatric patients by a prospective global registry.

Acknowledgements: We thank Thomy Tonia, ERS Senior Methodologist, for her methodological overview and Valérie Vaccaro, ERS Scientific Activities Project Lead, for organising the task force meetings. With thanks to Patrick Corkery, PHA Ireland and Gergely Meszaros, PHA Europe, who provided input on patient views and preferences via teleconferences, attendance at face to face meetings, and in writing. Mr Corkery and Mr Meszaros contributed to formulating and prioritising the key questions, identifying aspects important to patients, and in reviewing the final manuscript. Patrick Corkery underwent PEA surgery in Royal Papworth Hospital in January 2016, recovered and swam the English Channel in September 2017.

Conflict of interest: M. Delcroix reports grants and other (investigator, speaker and consultant fees received by the institution) from Actelion/J\&J, other (investigator, speaker and consultant fees received by the institution) from Bayer, other (speaker and consultant fees received by the institution) from MSD, other (investigator fees received by the institution) from Reata, other (investigator and consultant fees received by the institution) from Bellarophon, other (consultant fees received by the institution) from Acceleron, outside the submitted work. A. Torbicki reports grants and personal fees for lectures and consultancy from Actelion/Janssen, Bayer and MSD, personal fees for lectures from AOP, personal fees for lectures and consultancy from Pfizer, outside the submitted work. D. Gopalan reports other (speaker fees) from Actelion/J\&J, other (consultancy work and speaker fees) from Bayer, outside the submitted work. O. Sitbon reports grants, personal fees and non-financial support from Actelion Pharmaceuticals and MSD, grants from GlaxoSmithKline, personal fees from Bayer, Acceleron Pharmaceuticals, Gossamer Bio and Ferrer, outside the submitted work. F.A. Klok reports research grants from Bayer, Bristol-Myers Squibb, Boehringer Ingelheim, Daiichi-Sankyo, MSD and Actelion, the Dutch Heart Foundation and the Dutch Thrombosis association, outside the submitted work. I. Lang reports grants and personal fees from Actelion-Janssen and AOP Orphan Pharma, personal fees from Medtronic, Ferrer and United Therapeutics, outside the submitted work. D. Jenkins reports grants from Bayer, personal fees for advisory board work from Actelion, outside the submitted work. N.H. Kim reports personal fees for consultancy from Actelion, Bayer and Merck, outside the submitted work. M. Humbert reports grants and personal fees from Actelion and Bayer, personal fees from Acceleron, GSK, Merck, Novartis, AstraZeneca and Sanofi, outside the submitted work. X. Jais reports personal fees and non-financial support from Actelion and MSD, grants from Bayer, outside the submitted work. A. Vonk Noordegraaf is supported by the Netherlands CardioVascular Research Initiative (CVON-2012-08 PHAEDRA, CVON-2017-10 DOLPHIN-GENESIS) and the Netherlands Organization for Scientific Research (NWO-VICI: 918.16.610), has received speakers' money from Johnson \& Johnson and Ferrer in the past 3 years, and served as a member of the scientific advisory board of Morphogen-XI. J. Pepke-Zaba has received speaker fees and honoraria for consultations from Actelion, Merck and Bayer, and her institution received research and educational grants from Actelion and Merck. P. Brénot has nothing to disclose. P. Dorfmuller has nothing to disclose. E. Fadel has nothing to disclose. H-A. Ghofrani reports personal fees and other (consultancy fees) from Actelion, Bayer AG, GlaxoSmithKline, Novartis and Pfizer, other (consultancy fees) from Bellerophon Pulse Technologies and MSD, grants from Deutsche Forschungsgemeinschaft (DFG), during the conduct of the study. M.M. Hoeper reports personal fees for 
consultancy and lectures from Bayer AG, MSD, Actelion, Jansen, Acceleron and Pfizer, during the conduct of the study. P. Jansa reports other (investigator) from Actelion, personal fees and other (investigator) from Bayer Pharma AG and Reata Pharmaceuticals, personal fees from AOP and MSD, outside the submitted work. M. Madani reports personal fees for consultancy from Actelion and Wexler Surgical, outside the submitted work. H. Matsubara reports personal fees from Actelion Pharmaceuticals Japan, Ltd, AOP Orphan Pharmaceuticals AG, Bayer Yakuhin, Ltd, Pfizer Japan, Inc. Nippon Shinyaku, Co., Ltd, Kaneka Medix Corporation, GlaxoSmithKline Pharmaceuticals, Ltd and United Therapeutics Corporation, outside the submitted work. T. Ogo has nothing to disclose. E. Grünig reports fees for lectures and/or consultations from Actelion, Bayer AG, GSK, MSD, United Therapeutics and Pfizer, outside the submitted work. A. D'Armini reports personal fees from Actelion Phamaceuticals, Bayer AG and Merck Sharp \& Dohme, outside the submitted work. N. Galie reports grants and personal fees from Actelion and Janssen, personal fees from Pfizer and Ferrer, outside the submitted work. B. Meyer reports personal fees for lectures from Bayer AG, outside the submitted work. P. Corkery has nothing to disclose. G. Meszaros reports personal fees from Actelion Pharmaceuticals, outside the submitted work. E. Mayer reports personal fees for lectures and consultancy from Actelion, Bayer and MSD, during the conduct of the study. G. Simonneau reports personal fees and non-financial support from Actelion, Bayer and MSD, outside the submitted work.

Support statement: Travel support was provided by the International CTEPH Association (ICA) for its board members: M. Delcroix, E. Fadel, D. Jenkins, N. Kim, I. Lang, M. Madani, E. Mayer, H. Matsubara, J. Pepke-Zaba, and G. Simonneau. M. Humbert is supported by the Investissement d'Avenir programme managed by the French National Research Agency under the grant contract ANR-18-RHUS-0006 (DESTINATION 2024). The project was supported by European Respiratory Society grant TF 2018-04. Funding information for this article has been deposited with the Crossref Funder Registry.

\section{References}

1 Galiè N, Humbert M, Vachiéry J-L, et al. 2015 ESC/ERS Guidelines for the diagnosis and treatment of pulmonary hypertension: The Joint Task Force for the Diagnosis and Treatment of Pulmonary Hypertension of the European Society of Cardiology (ESC) and the European Respiratory Society (ERS). Eur Respir J 2015; 46: 903-975.

2 Taboada D, Pepke-Zaba J, Jenkins DP, et al. Outcome of pulmonary endarterectomy in symptomatic chronic thromboembolic disease. Eur Respir J 2014; 44: 1635-1645.

3 de Perrot M, Mayer E. Chronic thromboembolic pulmonary hypertension: do we need a new definition? Eur Respir J 2014; 44: 1401-1403.

4 Simonneau G, Montani D, Celermajer DS, et al. Haemodynamic definitions and updated clinical classification of pulmonary hypertension. Eur Respir J 2019; 53: 1801913.

5 van Kan C, van der Plas MN, Reesink HJ, et al. Hemodynamic and ventilatory responses during exercise in chronic thromboembolic disease. J Thorac Cardiovasc Surg 2016; 152: 763-771.

6 Held M, Kolb P, Grün M, et al. Functional characterization of patients with chronic thromboembolic disease. Respiration 2016; 91: 503-509.

7 Claeys M, Claessen G, La Gerche A, et al. Impaired cardiac reserve and abnormal vascular load limit exercise capacity in chronic thromboembolic disease. JACC Cardiovasc Imaging 2019; 12: 1444-1456.

8 Guth S, Wiedenroth $\mathrm{CB}$, Rieth $\mathrm{A}$, et al. Exercise right heart catheterisation before and after pulmonary endarterectomy in patients with chronic thromboembolic disease. Eur Respir J 2018; 52: 1800458.

9 Swietlik EM, Ruggiero A, Fletcher AJ, et al. Limitations of resting haemodynamics in chronic thromboembolic disease without pulmonary hypertension. Eur Respir J 2019; 53: 1801787.

10 Kovacs G, Herve P, Barbera JA, et al. An official European Respiratory Society statement: pulmonary haemodynamics during exercise. Eur Respir J 2017; 50: 1700578.

11 Schwaiblmair M, Faul C, von Scheidt W, et al. Detection of exercise-induced pulmonary arterial hypertension by cardiopulmonary exercise testing. Clin Cardiol 2012; 35: 548-553.

12 Held M, Grün M, Holl R, et al. Cardiopulmonary exercise testing to detect chronic thromboembolic pulmonary hypertension in patients with normal echocardiography. Respiration 2014; 87: 379-387.

13 Quezada CA, Jiménez D. Cardiopulmonary exercise testing with ventilatory gas analysis for evaluation of chronic thromboembolic pulmonary hypertension: unmasking its role after a therapeuthic intervention. Int $J$ Cardiol 2019; 296: 155-156.

14 Weatherald J, Farina S, Bruno N, et al. Cardiopulmonary exercise testing in pulmonary hypertension. Ann Am Thorac Soc 2017; 14: S84-S92.

15 Zhao Q-H, Wang L, Pudasaini B, et al. Cardiopulmonary exercise testing improves diagnostic specificity in patients with echocardiography-suspected pulmonary hypertension. Clin Cardiol 2017; 40: 95-101.

16 Pinkstaff SO, Burger CD, Daugherty J, et al. Cardiopulmonary exercise testing in patients with pulmonary hypertension: clinical recommendations based on a review of the evidence. Expert Rev Respir Med 2016; 10: 279-295.

17 Boulate D, Perros F, Dorfmüller P, et al. Pulmonary microvascular lesions regress in reperfused chronic thromboembolic pulmonary hypertension. J Heart Lung Transplant 2015; 34: 457-467.

18 Dorfmüller P, Günther S, Ghigna M-R, et al. Microvascular disease in chronic thromboembolic pulmonary hypertension: a role for pulmonary veins and systemic vasculature. Eur Respir J 2014; 44: 1275-1288.

19 Kim NH, Delcroix M, Jenkins DP, et al. Chronic thromboembolic pulmonary hypertension. J Am Coll Cardiol 2013; 62: Suppl. 25, D92-D99.

20 Kim NH, Delcroix M, Jais X, et al. Chronic thromboembolic pulmonary hypertension. Eur Respir J 2019; 53: 1801915.

21 Kan Y, Yuan L, Meeks JK, et al. The accuracy of V/Q SPECT in the diagnosis of pulmonary embolism: a meta-analysis. Acta Radiol 2015; 56: 565-572.

22 Phillips JJ, Straiton J, Staff RT. Planar and SPECT ventilation/perfusion imaging and computed tomography for the diagnosis of pulmonary embolism: a systematic review and meta-analysis of the literature, and cost and dose comparison. Eur J Radiol 2015; 84: 1392-1400. 

single-photon emission computed tomography versus computed tomography angiography - controversies, results, and recommendations from a systematic review. Semin Thromb Hemost 2016; 42: 833-845.

24 Soler X, Hoh CK, Test VJ, et al. Single photon emission computed tomography in chronic thromboembolic pulmonary hypertension. Respirology 2011; 16: 131-137.

25 Simanek M, Koranda P. The benefit of personalized hybrid SPECT/CT pulmonary imaging. Am J Nucl Med Mol Imaging 2016; 6: 215-222.

26 Derlin T, Kelting C, Hueper K, et al. Quantitation of perfused lung volume using hybrid SPECT/CT allows refining the assessment of lung perfusion and estimating disease extent in chronic thromboembolic pulmonary hypertension. Clin Nucl Med 2018; 43: e170-e177.

27 Roach PJ, Bailey DL, Schembri GP, et al. Transition from planar to SPECT V/Q scintigraphy: rationale, practicalities, and challenges. Semin Nucl Med 2010; 40: 397-407.

28 Bajc M, Neilly JB, Miniati M, et al. EANM guidelines for ventilation/perfusion scintigraphy: part 1. Pulmonary imaging with ventilation/perfusion single photon emission tomography. Eur J Nucl Med Mol Imaging 2009; 36: $1356-1370$.

29 Fuld MK, Halaweish AF, Haynes SE, et al. Pulmonary perfused blood volume with dual-energy CT as surrogate for pulmonary perfusion assessed with dynamic multidetector CT. Radiology 2013; 267: 747-756.

30 Giordano J, Khung S, Duhamel A, et al. Lung perfusion characteristics in pulmonary arterial hypertension $(\mathrm{PAH})$ and peripheral forms of chronic thromboembolic pulmonary hypertension (pCTEPH): dual-energy CT experience in 31 patients. Eur Radiol 2016; 27: 1631-1639.

31 Kim S-S, Hur J, Kim YJ, et al. Dual-energy CT for differentiating acute and chronic pulmonary thromboembolism: an initial experience. Int J Cardiovasc Imaging 2014; 30: 113-120.

32 Le Faivre J, Duhamel A, Khung S, et al. Impact of CT perfusion imaging on the assessment of peripheral chronic pulmonary thromboembolism: clinical experience in 62 patients. Eur Radiol 2016; 26: 4011-4020.

33 Takagi H, Ota H, Sugimura K, et al. Dual-energy CT to estimate clinical severity of chronic thromboembolic pulmonary hypertension: comparison with invasive right heart catheterization. Eur J Radiol 2016; 85: 1574-1580.

34 Meinel F, Graef A, Thierfelder K, et al. Automated quantification of pulmonary perfused blood volume by dual-energy CTPA in chronic thromboembolic pulmonary hypertension. Rofo 2014; 186: 151-156.

35 Renapurkar RD, Bolen MA, Shrikanthan S, et al. Comparative assessment of qualitative and quantitative perfusion with dual-energy CT and planar and SPECT-CT V/Q scanning in patients with chronic thromboembolic pulmonary hypertension. Cardiovasc Diagn Ther 2018; 8: 414-422.

36 Nakazawa T, Watanabe Y, Hori Y, et al. Lung perfused blood volume images with dual-energy computed tomography for chronic thromboembolic pulmonary hypertension: correlation to scintigraphy with single-photon emission computed tomography. J Comput Assist Tomogr 2011; 35: 590-595.

37 Masy M, Giordano J, Petyt G, et al. Dual-energy CT (DECT) lung perfusion in pulmonary hypertension: concordance rate with $\mathrm{V} / \mathrm{Q}$ scintigraphy in diagnosing chronic thromboembolic pulmonary hypertension (CTEPH). Eur Radiol 2018; 28: 5100-5110.

38 Dournes G, Verdier D, Montaudon M, et al. Dual-energy CT perfusion and angiography in chronic thromboembolic pulmonary hypertension: diagnostic accuracy and concordance with radionuclide scintigraphy. Eur Radiol 2014; 24: 42-51.

39 Johns CS, Swift AJ, Rajaram S, et al. Lung perfusion: MRI vs. SPECT for screening in suspected chronic thromboembolic pulmonary hypertension. J Magn Reson Imaging 2017; 46: 1693-1697.

40 Rajaram S, Swift AJ, Telfer A, et al. 3D contrast-enhanced lung perfusion MRI is an effective screening tool for chronic thromboembolic pulmonary hypertension: results from the ASPIRE Registry. Thorax 2013; 68: 677-678.

41 He J, Fang W, Lv B, et al. Diagnosis of chronic thromboembolic pulmonary hypertension: comparison of ventilation/perfusion scanning and multidetector computed tomography pulmonary angiography with pulmonary angiography. Nucl Med Commun 2012; 33: 459-463.

42 Rogberg AN, Gopalan D, Westerlund E, et al. Do radiologists detect chronic thromboembolic disease on computed tomography? Acta Radiol 2019; 60: 1576-1583.

43 Dong C, Zhou M, Liu D, et al. Diagnostic accuracy of computed tomography for chronic thromboembolic pulmonary hypertension: a systematic review and meta-analysis. PLoS One 2015; 10: e0126985.

44 Hinrichs J, Werncke T, Kaireit T, et al. C-arm computed tomography adds diagnostic information in patients with chronic thromboembolic pulmonary hypertension and a positive V/Q SPECT. Rofo 2017; 189: 49-56.

45 Hinrichs JB, von Falck C, Hoeper MM, et al. Pulmonary artery imaging in patients with chronic thromboembolic pulmonary hypertension: comparison of cone-beam CT and 64-row multidetector CT. J Vasc Interv Radiol 2016; 27: 361-368.

46 Fukuda T, Ogo T, Nakanishi N, et al. Evaluation of organized thrombus in distal pulmonary arteries in patients with chronic thromboembolic pulmonary hypertension using cone-beam computed tomography. Jpn J Radiol 2016; 34: 423-431.

47 Sugiyama M, Fukuda T, Sanda Y, et al. Organized thrombus in pulmonary arteries in patients with chronic thromboembolic pulmonary hypertension; imaging with cone beam computed tomography. Jpn J Radiol 2014; 32: $375-382$.

48 Hinrichs JB, Renne J, Hoeper MM, et al. Balloon pulmonary angioplasty: applicability of C-arm CT for procedure guidance. Eur Radiol 2016; 26: 4064-4071.

49 Ogo T, Fukuda T, Tsuji A, et al. Efficacy and safety of balloon pulmonary angioplasty for chronic thromboembolic pulmonary hypertension guided by cone-beam computed tomography and electrocardiogram-gated area detector computed tomography. Eur J Radiol 2017; 89: 270-276.

50 Maschke SK, Hinrichs JB, Renne J, et al. C-arm computed tomography (CACT)-guided balloon pulmonary angioplasty (BPA): evaluation of patient safety and peri- and post-procedural complications. Eur Radiol 2018; 29: $1276-1284$.

51 Ley S, Ley-Zaporozhan J, Pitton MB, et al. Diagnostic performance of state-of-the-art imaging techniques for morphological assessment of vascular abnormalities in patients with chronic thromboembolic pulmonary hypertension (CTEPH). Eur Radiol 2012; 22: 607-616. 

unenhanced proton MR imaging compared with CT pulmonary angiography in chronic thromboembolic pulmonary hypertension. Eur Radiol 2012; 22: 310-317.

53 Grothues F, Moon JC, Bellenger NG, et al. Interstudy reproducibility of right ventricular volumes, function, and mass with cardiovascular magnetic resonance. Am Heart J 2004; 147: 218-223.

54 Kreitner K-FJ, Ley S, Kauczor H-U, et al. Chronic thromboembolic pulmonary hypertension: pre- and postoperative assessment with breath-hold MR imaging techniques. Radiology 2004; 232: 535-543.

55 Mauritz GJ, Vonk-Noordegraaf A, Kind T. Pulmonary endarterectomy normalizes interventricular dyssynchrony and right ventricular systolic wall stress. J Cardiovasc Magn Reson 2012; 14: 5.

56 Berman M, Gopalan D, Sharples L, et al. Right ventricular reverse remodeling after pulmonary endarterectomy: magnetic resonance imaging and clinical and right heart catheterization assessment. Pulm Circ 2014; 4: 36-44.

57 Fukui S, Ogo T, Morita Y, et al. Right ventricular reverse remodelling after balloon pulmonary angioplasty. Eur Respir J 2014; 43: 1394-1402.

58 Roller FC, Wiedenroth C, Breithecker A, et al. Native T1 mapping and extracellular volume fraction measurement for assessment of right ventricular insertion point and septal fibrosis in chronic thromboembolic pulmonary hypertension. Eur Radiol 2017; 27: 1980-1991.

59 Roeleveld RJ, Marcus JT, Faes TJC, et al. Interventricular septal configuration at MR imaging and pulmonary arterial pressure in pulmonary hypertension. Radiology 2005; 234: 710-717.

60 Sanz J, Kuschnir P, Rius T, et al. Pulmonary arterial hypertension: noninvasive detection with phase-contrast MR imaging. Radiology 2007; 243: 70-79.

61 Kreitner K-F, Wirth GM, Krummenauer F, et al. Noninvasive assessment of pulmonary hemodynamics in patients with chronic thromboembolic pulmonary hypertension by high temporal resolution phase-contrast MRI: correlation with simultaneous invasive pressure recordings. Circ Cardiovasc Imaging 2013; 6: 722-729.

62 García-Alvarez A, Fernández-Friera L, Mirelis JG, et al. Non-invasive estimation of pulmonary vascular resistance with cardiac magnetic resonance. Eur Heart J 2011; 32: 2438-2445.

63 Reiter G, Reiter U, Kovacs G, et al. Blood flow vortices along the main pulmonary artery measured with MR imaging for diagnosis of pulmonary hypertension. Radiology 2015; 275: 71-79.

64 Reiter G, Reiter U, Kovacs G, et al. Magnetic resonance-derived 3-dimensional blood flow patterns in the main pulmonary artery as a marker of pulmonary hypertension and a measure of elevated mean pulmonary arterial pressure. Circ Cardiovasc Imaging 2008; 1: 23-30.

65 Delcroix M, Kerr K, Fedullo P. Chronic thromboembolic pulmonary hypertension. Epidemiology and risk factors. Ann Am Thorac Soc 2016; 13: Suppl. 3, S201-S206.

66 Escribano-Subias P, Blanco I, López-Meseguer M, et al. Survival in pulmonary hypertension in Spain: insights from the Spanish registry. Eur Respir J 2012; 40: 596-603.

67 Gibbs S. National Audit of Pulmonary Hypertension, Great Britain, 2018-19. October 24, 2019. https://files. digital.nhs.uk/BA/4EF20E/NAPH\%2010AR\%20-\%20Main\%20Report.pdf Date last accessed: February 24, 2020.

68 Gall H, Hoeper MM, Richter MJ, et al. An epidemiological analysis of the burden of chronic thromboembolic pulmonary hypertension in the USA, Europe and Japan. Eur Respir Rev 2017; 26: 160121.

69 Cottin V, Avot D, Lévy-Bachelot L, et al. Identifying chronic thromboembolic pulmonary hypertension through the French national hospital discharge database. PLoS One 2019; 14: e0214649.

70 Moser KM, Auger WR, Fedullo PF, et al. Chronic thromboembolic pulmonary hypertension: clinical picture and surgical treatment. Eur Respir J 1992; 5: 334-342.

71 Pepke-Zaba J, Delcroix M, Lang I, et al. Chronic thromboembolic pulmonary hypertension (CTEPH): results from an international prospective registry. Circulation 2011; 124: 1973-1981.

72 Lang IM, Madani M. Update on chronic thromboembolic pulmonary hypertension. Circulation 2014; 130: 508-518.

73 Ogawa A, Satoh T, Fukuda T, et al. Balloon pulmonary angioplasty for chronic thromboembolic pulmonary hypertension: results of a multicenter registry. Circ Cardiovasc Qual Outcomes 2017; 10: e004029.

74 Chausheva S, Naito A, Ogawa A, et al. Chronic thromboembolic pulmonary hypertension in Austria and Japan. J Thorac Cardiovasc Surg 2019; 158: 604-614.

75 Pengo V, Lensing AWA, Prins $\mathrm{MH}$, et al. Incidence of chronic thromboembolic pulmonary hypertension after pulmonary embolism. N Engl J Med 2004; 350: 2257-2264.

76 Golpe R, Pérez-de-Llano LA, Castro-Añón O, et al. Right ventricle dysfunction and pulmonary hypertension in hemodynamically stable pulmonary embolism. Respir Med 2010; 104: 1370-1376.

77 Guérin L, Couturaud F, Parent F, et al. Prevalence of chronic thromboembolic pulmonary hypertension after acute pulmonary embolism. Prevalence of CTEPH after pulmonary embolism. Thromb Haemost 2014; 112: 598-605.

78 Simonneau G, Hoeper MM. Evaluation of the incidence of rare diseases: difficulties and uncertainties, the example of chronic thromboembolic pulmonary hypertension. Eur Respir J 2017; 49: 1602522.

79 Ende-Verhaar YM, Cannegieter SC, Vonk-Noordegraaf A, et al. Incidence of chronic thromboembolic pulmonary hypertension after acute pulmonary embolism: a contemporary view of the published literature. Eur Respir J 2017; 49: 1601792.

80 Coquoz N, Weilenmann D, Stolz D, et al. Multicentre observational screening survey for the detection of CTEPH following pulmonary embolism. Eur Respir J 2018; 51: 1702505.

81 Wartski M, Collignon M-A. Incomplete recovery of lung perfusion after 3 months in patients with acute pulmonary embolism treated with antithrombotic agents. THESEE Study Group. Tinzaparin ou Heparin Standard: Evaluation dans l'Embolie Pulmonaire Study. J Nucl Med 2000; 41: 1043-1048.

82 Nijkeuter M, Hovens MMC, Davidson BL, et al. Resolution of thromboemboli in patients with acute pulmonary embolism: a systematic review. Chest 2006; 129: 192-197.

83 Sanchez O, Helley D, Couchon S, et al. Perfusion defects after pulmonary embolism: risk factors and clinical significance. J Thromb Haemost 2010; 8: 1248-1255.

84 Meneveau N, Ider O, Seronde M-F, et al. Long-term prognostic value of residual pulmonary vascular obstruction at discharge in patients with intermediate- to high-risk pulmonary embolism. Eur Heart J 2013; 34: 693-701. 

limitation at 12 months: results of the ELOPE Study. Res Pract Thromb Haemost 2018; 2: 670-677. prognosis and evolution of hemodynamic and respiratory data. Chest 1982; 81: 151-158.

87 Lewczuk J, Piszko P, Jagas J, et al. Prognostic factors in medically treated patients with chronic pulmonary embolism. Chest 2001; 119: 818-823.

88 Prandoni P, Ageno W, Mumoli N, et al. Recanalization rate in patients with proximal vein thrombosis treated with the direct oral anticoagulants. Thromb Res 2017; 153: 97-100.

89 Piazza G, Mani V, Goldhaber SZ, et al. Magnetic resonance venography to assess thrombus resolution with edoxaban monotherapy versus parenteral anticoagulation/warfarin for symptomatic deep vein thrombosis: a multicenter feasibility study. Vasc Med 2016; 21: 361-368.

90 Bunclark K, Newnham M, Chiu Y-D, et al. A multicenter study of anticoagulation in operable chronic thromboembolic pulmonary hypertension. J Thromb Haemost 2020; 18: 114-122.

91 Houghton DE, Lekah A, Macedo TA, et al. Resolution of acute lower extremity deep vein thrombosis with rivaroxaban compared to warfarin. J Thromb Thrombolysis 2020; 49: 199-205.

92 Konstantinides SV, Meyer G, Becattini C, et al. 2019 ESC Guidelines for the diagnosis and management of acute pulmonary embolism developed in collaboration with the European Respiratory Society (ERS): the task force for the diagnosis and management of acute pulmonary embolism of the European Society of Cardiology (ESC). Eur Respir J 2019; 54: 1901647.

93 Konstantinides SV, Meyer G, Becattini C, et al. 2019 ESC Guidelines for the diagnosis and management of acute pulmonary embolism developed in collaboration with the European Respiratory Society (ERS). Eur Heart J 2020; 41: 543-603.

94 Pengo V, Denas G, Zoppellaro G, et al. Rivaroxaban vs warfarin in high-risk patients with antiphospholipid syndrome. Blood 2018; 132: 1365-1371.

95 Moll S, Crona DJ, Martin K. Direct oral anticoagulants in extremely obese patients: OK to use? Res Pract Thromb Haemost 2019; 3: 152-155.

96 Ingrasciotta Y, Crisafulli S, Pizzimenti V, et al. Pharmacokinetics of new oral anticoagulants: implications for use in routine care. Expert Opin Drug Metab Toxicol 2018; 14: 1057-1069.

97 Bonderman D, Jakowitsch J, Adlbrecht C, et al. Medical conditions increasing the risk of chronic thromboembolic pulmonary hypertension. Thromb Haemost 2005; 93: 512-516.

98 Lang I, Simonneau G, Pepke-Zaba J, et al. Factors associated with diagnosis and operability of chronic thromboembolic pulmonary hypertension. A case-control study. Thromb Haemost 2013; 110: 83-91.

99 Delcroix M, Lang I, Pepke-Zaba J, et al. Long-term outcome of patients with chronic thromboembolic pulmonary hypertension: results from an international prospective registry. Circulation 2016; 133: 859-871.

100 Ende-Verhaar YM, Huisman MV, Klok FA. To screen or not to screen for chronic thromboembolic pulmonary hypertension after acute pulmonary embolism. Thromb Res 2017; 151: 1-7.

101 Ende-Verhaar YM, van den Hout WB, Bogaard H-J, et al. Healthcare utilization in chronic thromboembolic pulmonary hypertension after acute pulmonary embolism. J Thromb Haemost 2018; 16: 2168-2174.

102 Klok FA, Barco S, Konstantinides SV, et al. Determinants of diagnostic delay in chronic thromboembolic pulmonary hypertension: results from the European CTEPH Registry. Eur Respir J 2018; 52: 1801687.

103 Jensen KW, Kerr KM, Fedullo PF, et al. Pulmonary hypertensive medical therapy in chronic thromboembolic pulmonary hypertension before pulmonary thromboendarterectomy. Circulation 2009; 120: 1248-1254.

104 Nishiyama KH, Saboo SS, Tanabe Y, et al. Chronic pulmonary embolism: diagnosis. Cardiovasc Diagn Ther 2018; 8: 253-271.

105 Rajaram S, Swift AJ, Condliffe R, et al. CT features of pulmonary arterial hypertension and its major subtypes: a systematic CT evaluation of 292 patients from the ASPIRE Registry. Thorax 2015; 70: 382-387.

106 Ende-Verhaar YM, Meijboom LJ, Kroft LJM, et al. Usefulness of standard computed tomography pulmonary angiography performed for acute pulmonary embolism for identification of chronic thromboembolic pulmonary hypertension: results of the InShape III study. J Heart Lung Transplant 2019; 38: 731-738.

107 McIntyre KM, Sasahara AA. The hemodynamic response to pulmonary embolism in patients without prior cardiopulmonary disease. Am J Cardiol 1971; 28: 288-294.

108 Klok FA, Delcroix M, Bogaard H-J. Chronic thromboembolic pulmonary hypertension from the perspective of patients with pulmonary embolism. J Thromb Haemost 2018; 16: 1040-1051.

109 Sista AK, Klok FA. Late outcomes of pulmonary embolism: the post-PE syndrome. Thromb Res 2018; 164: $157-162$.

110 Sista AK, Miller LE, Kahn SR, et al. Persistent right ventricular dysfunction, functional capacity limitation, exercise intolerance, and quality of life impairment following pulmonary embolism: systematic review with meta-analysis. Vasc Med 2017; 22: 37-43.

111 Klok FA, van der Hulle T, den Exter PL, et al. The post-PE syndrome: a new concept for chronic complications of pulmonary embolism. Blood Rev 2014; 28: 221-226.

112 Klok FA, Dzikowska-Diduch O, Kostrubiec M, et al. Derivation of a clinical prediction score for chronic thromboembolic pulmonary hypertension after acute pulmonary embolism. J Thromb Haemost 2016; 14: $121-128$.

113 Wittram C. How I do it: CT pulmonary angiography. AJR Am J Roentgenol 2007; 188: 1255-1261.

114 Helmersen D, Provencher S, Hirsch AM, et al. Diagnosis of chronic thromboembolic pulmonary hypertension: a Canadian Thoracic Society clinical practice guideline update. Can J Respir Crit Care Sleep Med 2019; 3: 177-198.

115 Klok FA, van Kralingen KW, van Dijk APJ, et al. Prevalence and potential determinants of exertional dyspnea after acute pulmonary embolism. Respir Med 2010; 104: 1744-1749.

116 Kahn SR, Akaberi A, Granton JT, et al. Quality of life, dyspnea, and functional exercise capacity following a first episode of pulmonary embolism: results of the ELOPE cohort study. Am J Med 2017; 130: 990.e9-990.e21.

117 Kahn SR, Hirsch AM, Akaberi A, et al. Functional and exercise limitations after a first episode of pulmonary embolism: results of the ELOPE prospective cohort study. Chest 2017; 151: 1058-1068.

118 Sznajder M, Dzikowska-Diduch O, Kurnicka K, et al. Increased systemic arterial stiffness in patients with chronic thromboembolic pulmonary hypertension. Cardiol J 2020; 27: 742-748. 
119 Pietra GG, Capron F, Stewart S, et al. Pathologic assessment of vasculopathies in pulmonary hypertension. J Am Coll Cardiol 2004; 43: 25S-32S.

120 Humbert M, Guignabert C, Bonnet S, et al. Pathology and pathobiology of pulmonary hypertension: state of the art and research perspectives. Eur Respir J 2019; 53: 1801887.

121 Moser KM, Bloor CM. Pulmonary vascular lesions occurring in patients with chronic major vessel thromboembolic pulmonary hypertension. Chest 1993; 103: 685-692.

122 Gerges C, Gerges M, Friewald R, et al. Microvascular disease in chronic thromboembolic pulmonary hypertension: hemodynamic phenotyping and histomorphometric assessment. Circulation 2020; 141: 376-386.

123 Noly P-E, Guihaire J, Coblence M, et al. Chronic thromboembolic pulmonary hypertension and assessment of right ventricular function in the piglet. J Vis Exp 2015; 105: e53133.

124 Tsai H-Y, Chin C-S, Tsai I-C. Imaging as a biomarker in Behçet disease: prognostic and therapeutic implications of serial pulmonary CT angiography. AJR Am J Roentgenol 2011; 196: W105-W106.

125 Perino MG, Moldobaeva A, Jenkins J, et al. Chemokine localization in bronchial angiogenesis. PLoS One 2013; 8: e66432.

126 Mercier O, Fadel E. Chronic thromboembolic pulmonary hypertension: animal models. Eur Respir J 2013; 41: $1200-1206$

127 Yi ES, Kim H, Ahn H, et al. Distribution of obstructive intimal lesions and their cellular phenotypes in chronic pulmonary hypertension. A morphometric and immunohistochemical study. Am J Respir Crit Care Med 2000; 162: $1577-1586$.

128 Galambos C, Sims-Lucas S, Abman SH, et al. Intrapulmonary bronchopulmonary anastomoses and plexiform lesions in idiopathic pulmonary arterial hypertension. Am J Respir Crit Care Med 2016; 193: 574-576.

129 Ghigna M-R, Guignabert C, Montani D, et al. BMPR2 mutation status influences bronchial vascular changes in pulmonary arterial hypertension. Eur Respir J 2016; 48: 1668-1681.

130 Azarian R, Wartski M, Collignon MA, et al. Lung perfusion scans and hemodynamics in acute and chronic pulmonary embolism. J Nucl Med 1997; 38: 980-983.

131 Kim NHS, Fesler P, Channick RN, et al. Preoperative partitioning of pulmonary vascular resistance correlates with early outcome after thromboendarterectomy for chronic thromboembolic pulmonary hypertension. Circulation 2004; 109: 18-22.

132 Toshner M, Suntharalingam J, Fesler $\mathrm{P}$, et al. Occlusion pressure analysis role in partitioning of pulmonary vascular resistance in CTEPH. Eur Respir J 2012; 40: 612-617.

133 Simonneau G, Torbicki A, Dorfmüller P, et al. The pathophysiology of chronic thromboembolic pulmonary hypertension. Eur Respir Rev 2017; 26: 160112.

134 Ley S, Kreitner K-F, Morgenstern I, et al. Bronchopulmonary shunts in patients with chronic thromboembolic pulmonary hypertension: evaluation with helical CT and MR imaging. AJR Am J Roentgenol 2002; 179: 1209-1215.

135 Miller F, Coulden RA, Sonnex E, et al. The use of MR flow mapping in the assessment of pulmonary artery blood flow following pulmonary thrombo-endarterectomy. Radiological Society of North America 2003 Scientific Assembly and Annual Meeting. December 2003: 426P.

136 Reiter U, Reiter G, Kovacs G, et al. Evaluation of elevated mean pulmonary arterial pressure based on magnetic resonance 4D velocity mapping: comparison of visualization techniques. PLoS One 2013; 8: e82212.

137 Ota H, Sugimura K, Miura M, et al. Four-dimensional flow magnetic resonance imaging visualizes drastic change in vortex flow in the main pulmonary artery after percutaneous transluminal pulmonary angioplasty in a patient with chronic thromboembolic pulmonary hypertension. Eur Heart J 2015; 36: 1630.

138 Polanowski T, Kurzyna M, Kuca P, et al. Róznice w hemodynamice pierwotnego i zatorowego nadciśnienia płucnego. [Differences in hemodynamics of thromboembolic and primary pulmonary hypertension]. Pol Arch Med Wewn 2000; 104: 741-745.

139 Quarck R, Nawrot T, Meyns B, et al. C-reactive protein: a new predictor of adverse outcome in pulmonary arterial hypertension. J Am Coll Cardiol 2009; 53: 1211-1218.

140 Axell RG, Messer SJ, White PA, et al. Ventriculo-arterial coupling detects occult RV dysfunction in chronic thromboembolic pulmonary vascular disease. Physiol Rep 2017; 5: e13227.

141 MacKenzie Ross RV, Toshner MR, Soon E, et al. Decreased time constant of the pulmonary circulation in chronic thromboembolic pulmonary hypertension. Am J Physiol Heart Circ Physiol 2013; 305: H259-H264.

142 Menzel T, Wagner S, Kramm T, et al. Pathophysiology of impaired right and left ventricular function in chronic embolic pulmonary hypertension: changes after pulmonary thromboendarterectomy. Chest 2000; 118: 897-903.

143 Bonderman D, Martischnig AM, Vonbank K, et al. Right ventricular load at exercise is a cause of persistent exercise limitation in patients with normal resting pulmonary vascular resistance after pulmonary endarterectomy. Chest 2011; 139: 122-127.

144 Jenkins D, Madani M, Fadel E, et al. Pulmonary endarterectomy in the management of chronic thromboembolic pulmonary hypertension. Eur Respir Rev 2017; 26: 160111.

145 Mayer E, Jenkins D, Lindner J, et al. Surgical management and outcome of patients with chronic thromboembolic pulmonary hypertension: results from an international prospective registry. J Thorac Cardiovasc Surg 2011; 141: 702-710.

146 Boulate D, Mercier O, Mussot S, et al. Extracorporeal life support after pulmonary endarterectomy as a bridge to recovery or transplantation: lessons from 31 consecutive patients. Ann Thorac Surg 2016; 102: 260-268.

147 Madani M, Ogo T, Simonneau G. The changing landscape of chronic thromboembolic pulmonary hypertension management. Eur Respir Rev 2017; 26: 170105.

148 Kawakami T, Ogawa A, Miyaji K, et al. Novel angiographic classification of each vascular lesion in chronic thromboembolic pulmonary hypertension based on selective angiogram and results of balloon pulmonary angioplasty. Circ Cardiovasc Interv 2016; 9: e003318.

149 D'Armini AM, Morsolini M, Mattiucci G, et al. Pulmonary endarterectomy for distal chronic thromboembolic pulmonary hypertension. J Thorac Cardiovasc Surg 2014; 148: 1005-1011.

150 Hsieh WC, Jansa P, Huang WC, et al. Residual pulmonary hypertension after pulmonary endarterectomy: a meta-analysis. J Thorac Cardiovasc Surg 2018; 156: 1275-1287. 
151 Madani MM, Auger WR, Pretorius V, et al. Pulmonary endarterectomy: recent changes in a single institution's experience of more than 2,700 patients. Ann Thorac Surg 2012; 94: 97-103.

152 Cannon JE, Su L, Kiely DG, et al. Dynamic risk stratification of patient long-term outcome after pulmonary endarterectomy: results from the United Kingdom national cohort. Circulation 2016; 133: 1761-1771.

153 Vuylsteke A, Sharples L, Charman G, et al. Circulatory arrest versus cerebral perfusion during pulmonary endarterectomy surgery (PEACOG): a randomised controlled trial. Lancet 2011; 378: 1379-1387.

154 Corsico AG, D'Armini AM, Cerveri I, et al. Long-term outcome after pulmonary endarterectomy. Am J Respir Crit Care Med 2008; 178: 419-424.

155 Kramm T, Eberle B, Guth S, et al. Inhaled iloprost to control residual pulmonary hypertension following pulmonary endarterectomy. Eur J Cardiothorac Surg 2005; 28: 882-888.

156 Ghofrani HA, D'Armini AM, Grimminger F, et al. Riociguat for the treatment of chronic thromboembolic pulmonary hypertension. N Engl J Med 2013; 369: 319-329.

157 Shimura N, Kataoka M, Inami T, et al. Additional percutaneous transluminal pulmonary angioplasty for residual or recurrent pulmonary hypertension after pulmonary endarterectomy. Int J Cardiol 2015; 183: 138-142.

158 Ali JM, Dunning J, Ng C, et al. The outcome of reoperative pulmonary endarterectomy surgery. Interact Cardiovasc Thorac Surg 2018; 26: 932-937.

159 Mizoguchi H, Ogawa A, Munemasa M, et al. Refined balloon pulmonary angioplasty for inoperable patients with chronic thromboembolic pulmonary hypertension. Circ Cardiovasc Interv 2012; 5: 748-755.

160 Kataoka M, Inami T, Hayashida K, et al. Percutaneous transluminal pulmonary angioplasty for the treatment of chronic thromboembolic pulmonary hypertension. Circ Cardiovasc Interv 2012; 5: 756-762.

161 Sugimura K, Fukumoto Y, Satoh K, et al. Percutaneous transluminal pulmonary angioplasty markedly improves pulmonary hemodynamics and long-term prognosis in patients with chronic thromboembolic pulmonary hypertension. Circ J 2012; 76: 485-488.

162 Lang I, Meyer BC, Ogo T, et al. Balloon pulmonary angioplasty in chronic thromboembolic pulmonary hypertension. Eur Respir Rev 2017; 26: 160119.

163 Wiedenroth CB, Liebetrau C, Breithecker A, et al. Combined pulmonary endarterectomy and balloon pulmonary angioplasty in patients with chronic thromboembolic pulmonary hypertension. J Heart Lung Transplant 2016; 35: 591-596.

164 Ogo T. Balloon pulmonary angioplasty for inoperable chronic thromboembolic pulmonary hypertension. Curr Opin Pulm Med 2015; 21: 425-431.

165 Zoppellaro G, Badawy MR, Squizzato A, et al. Balloon pulmonary angioplasty in patients with chronic thromboembolic pulmonary hypertension - a systematic review and meta-analysis. Circ J 2019; 83: 1660-1667.

166 Darocha S, Pietura R, Pietrasik A, et al. Improvement in quality of life and hemodynamics in chronic thromboembolic pulmonary hypertension treated with balloon pulmonary angioplasty. Circ J 2017; 81: 552-557.

167 Ogawa A, Matsubara H. After the dawn - balloon pulmonary angioplasty for patients with chronic thromboembolic pulmonary hypertension. Circ J 2018; 82: 1222-1230.

168 Fukui S, Ogo T, Goto Y, et al. Exercise intolerance and ventilatory inefficiency improve early after balloon pulmonary angioplasty in patients with inoperable chronic thromboembolic pulmonary hypertension. Int $J$ Cardiol 2015; 180: 66-68.

169 Olsson KM, Wiedenroth CB, Kamp J-C, et al. Balloon pulmonary angioplasty for inoperable patients with chronic thromboembolic pulmonary hypertension: the initial German experience. Eur Respir J 2017; 49: 1602409.

170 Brenot $\mathrm{P}$, Jaïs $\mathrm{X}$, Taniguchi $\mathrm{Y}$, et al. French experience of balloon pulmonary angioplasty for chronic thromboembolic pulmonary hypertension. Eur Respir J 2019; 53: 1802095.

171 Feinstein JA, Goldhaber SZ, Lock JE, et al. Balloon pulmonary angioplasty for treatment of chronic thromboembolic pulmonary hypertension. Circulation 2001; 103: 10-13.

172 Ejiri K, Ogawa A, Fujii S, et al. Vascular injury is a major cause of lung injury after balloon pulmonary angioplasty in patients with chronic thromboembolic pulmonary hypertension. Circ Cardiovasc Interv 2018; 11: e005884.

173 Inami $\mathrm{T}$, Kataoka $\mathrm{M}$, Shimura $\mathrm{N}$, et al. Incidence, avoidance, and management of pulmonary artery injuries in percutaneous transluminal pulmonary angioplasty. Int J Cardiol 2015; 201: 35-37.

174 Inami T, Kataoka M, Yanagisawa R, et al. Long-term outcomes after percutaneous transluminal pulmonary angioplasty for chronic thromboembolic pulmonary hypertension. Circulation 2016; 134: 2030-2032.

175 Aoki T, Sugimura K, Tatebe S, et al. Comprehensive evaluation of the effectiveness and safety of balloon pulmonary angioplasty for inoperable chronic thrombo-embolic pulmonary hypertension: long-term effects and procedure-related complications. Eur Heart J 2017; 38: 3152-3159.

176 Taniguchi $\mathrm{Y}$, Jaïs $\mathrm{X}$, Jevnikar $\mathrm{M}$, et al. Predictors of survival in patients with not-operated chronic thromboembolic pulmonary hypertension. J Heart Lung Transplant 2019; 38: 833-842.

177 Humbert M, Simonneau G, Pittrow D, et al. Safety of riociguat in patients with pulmonary arterial hypertension and chronic thromboembolic pulmonary hypertension with concomitant novel oral anticoagulants or vitamin $\mathrm{K}$ antagonist use: data from the EXPERT Registry. Am J Respir Crit Care Med 2020; 201: A6043.

178 Jeong I, Fernandes T, Alotaibi M, et al. Direct oral anticoagulant use and thrombus detection in patients with chronic thromboembolic pulmonary hypertension referred for pulmonary thromboendarterectomy. Eur Respir $J$ 2019; 54: OA5161.

179 Skoro-Sajer N, Hack N, Sadushi-Koliçi R, et al. Pulmonary vascular reactivity and prognosis in patients with chronic thromboembolic pulmonary hypertension: a pilot study. Circulation 2009; 119: 298-305.

180 Suntharalingam J, Hughes RJ, Goldsmith K, et al. Acute haemodynamic responses to inhaled nitric oxide and intravenous sildenafil in distal chronic thromboembolic pulmonary hypertension (CTEPH). Vascul Pharmacol 2007; 46: 449-455.

181 Suntharalingam J, Treacy CM, Doughty NJ, et al. Long-term use of sildenafil in inoperable chronic thromboembolic pulmonary hypertension. Chest 2008; 134: 229-236.

182 Jaïs X, D'Armini AM, Jansa P, et al. Bosentan for treatment of inoperable chronic thromboembolic pulmonary hypertension: BENEFiT (Bosentan Effects in iNopErable Forms of chronIc Thromboembolic pulmonary hypertension), a randomized, placebo-controlled trial. J Am Coll Cardiol 2008; 52: 2127-2134. 
183 Ghofrani HA, Simonneau G, D'Armini AM, et al. Macitentan for the treatment of inoperable chronic thromboembolic pulmonary hypertension (MERIT-1): results from the multicentre, phase 2, randomised, double-blind, placebo-controlled study. Lancet Respir Med 2017; 5: 785-794.

184 Sadushi-Koliçi R, Jansa P, Kopeć G, et al. Subcutaneous treprostinil for the treatment of severe non-operable chronic thromboembolic pulmonary hypertension $(\mathrm{CTREPH})$ : a double-blind, phase 3, randomised controlled trial. Lancet Respir Med 2019; 7: 239-248.

185 Gabrielly $\mathrm{M}$, Bourlier D, Taniguchi $\mathrm{Y}$, et al. Initial dual oral combination therapy in inoperable chronic thromboembolic pulmonary hypertension (CTEPH). Eur Respir J 2018; 52: PA3053.

186 Tromeur C, Jaïs X, Mercier O, et al. Factors predicting outcome after pulmonary endarterectomy. PLoS One 2018; 13: e0198198.

187 Nagaya N, Sasaki N, Ando M, et al. Prostacyclin therapy before pulmonary thromboendarterectomy in patients with chronic thromboembolic pulmonary hypertension. Chest 2003; 123: 338-343.

188 Bresser P, Fedullo PF, Auger WR, et al. Continuous intravenous epoprostenol for chronic thromboembolic pulmonary hypertension. Eur Respir J 2004; 23: 595-600.

189 Reesink HJ, Surie S, Kloek JJ, et al. Bosentan as a bridge to pulmonary endarterectomy for chronic thromboembolic pulmonary hypertension. J Thorac Cardiovasc Surg 2010; 139: 85-91.

190 Castro MA, Piloto B, Dos Santos Fernandes CJC, et al. Use of medical therapies before pulmonary endarterectomy in chronic thromboembolic pulmonary hypertension patients with severe hemodynamic impairment. PLoS One 2020; 15: e0233063. PMID 32442171.

191 Kawashima T, Yoshitake A, Kawakami T, et al. Two-stage treatment using balloon pulmonary angioplasty and pulmonary endarterectomy in a patient with chronic thromboembolic pulmonary hypertension. Ann Vasc Surg 2018; 49: 315.e5-315.e7.

192 Yanaka K, Nakayama K, Shinke T, et al. Sequential hybrid therapy with pulmonary endarterectomy and additional balloon pulmonary angioplasty for chronic thromboembolic pulmonary hypertension. J Am Heart Assoc 2018; 7: e008838.

193 Araszkiewicz A, Darocha S, Pietrasik A, et al. Balloon pulmonary angioplasty for the treatment of residual or recurrent pulmonary hypertension after pulmonary endarterectomy. Int J Cardiol 2019; 278: 232-237.

194 Nakamura M, Sunagawa O, Tsuchiya $\mathrm{H}$, et al. Rescue balloon pulmonary angioplasty under veno-arterial extracorporeal membrane oxygenation in a patient with acute exacerbation of chronic thromboembolic pulmonary hypertension. Int Heart J 2015; 56: 116-120.

195 Collaud S, Brenot P, Mercier O, et al. Rescue balloon pulmonary angioplasty for early failure of pulmonary endarterectomy: The earlier the better? Int J Cardiol 2016; 222: 39-40.

196 Wiedenroth CB, Ghofrani HA, Adameit MSD, et al. Sequential treatment with riociguat and balloon pulmonary angioplasty for patients with inoperable chronic thromboembolic pulmonary hypertension. Pulm Circ 2018; 8: 2045894018783996.

197 Mereles D, Ehlken N, Kreuscher S, et al. Exercise and respiratory training improve exercise capacity and quality of life in patients with severe chronic pulmonary hypertension. Circulation 2006; 114: 1482-1489.

198 Ehlken N, Lichtblau M, Klose H, et al. Exercise training improves peak oxygen consumption and haemodynamics in patients with severe pulmonary arterial hypertension and inoperable chronic thrombo-embolic pulmonary hypertension: a prospective, randomized, controlled trial. Eur Heart J 2016; 37: $35-44$.

199 Handoko ML, De Man FS, Happé CM, et al. Opposite effects of training in rats with stable and progressive pulmonary hypertension. Circulation 2009; 120: 42-49.

200 Zhai $\mathrm{Z}$, Murphy $\mathrm{K}$, Tighe $\mathrm{H}$, et al. Differences in ventilatory inefficiency between pulmonary arterial hypertension and chronic thromboembolic pulmonary hypertension. Chest 2011; 140: 1284-1291.

201 Nagel C, Prange F, Guth S, et al. Exercise training improves exercise capacity and quality of life in patients with inoperable or residual chronic thromboembolic pulmonary hypertension. PLoS One 2012; 7: e41603.

202 Inagaki $\mathrm{T}$, Terada J, Tanabe $\mathrm{N}$, et al. Home-based pulmonary rehabilitation in patients with inoperable or residual chronic thromboembolic pulmonary hypertension: a preliminary study. Respir Investig 2014; 52: 357-364.

203 La Rovere MT, Pinna GD, Pin M, et al. Exercise training after pulmonary endarterectomy for patients with chronic thromboembolic pulmonary hypertension. Respiration 2019; 97: 234-241.

204 Fukui S, Ogo T, Takaki H, et al. Efficacy of cardiac rehabilitation after balloon pulmonary angioplasty for chronic thromboembolic pulmonary hypertension. Heart 2016; 102: 1403-1409.

205 Opitz I, Kirschner MB. Molecular research in chronic thromboembolic pulmonary hypertension. Int J Mol Sci 2019; 20: 784

206 Hemnes AR, Beck GJ, Newman JH, et al. PVDOMICS: a multi-center study to improve understanding of pulmonary vascular disease through phenomics. Circ Res 2017; 121: 1136-1139.

207 Newnham M, South K, Bleda M, et al. The ADAMTS13-VWF axis is dysregulated in chronic thromboembolic pulmonary hypertension. Eur Respir J 2019; 53: 1801805.

208 Heresi GA, Mey JT, Bartholomew JR, et al. Plasma metabolomic profile in chronic thromboembolic pulmonary hypertension. Pulm Circ 2020; 10: 2045894019890553.

209 Madani MM, Wittine LM, Auger WR, et al. Chronic thromboembolic pulmonary hypertension in pediatric patients. J Thorac Cardiovasc Surg 2011; 141: 624-630.

210 Kumbasar U, Aypar E, Karagöz T, et al. Pulmonary thromboendarterectomy in pediatric patients: report of three cases. Turk J Pediatr 2018; 60: 604-607.

211 Verbelen T, Cools B, Fejzic Z, et al. Pulmonary endarterectomy in a 12-year-old boy with multiple comorbidities. Pulm Circ 2019; 9: 2045894019886249. 